\title{
Non-uniformizable sets with countable cross-sections on a given level of the projective hierarchy*
}

\author{
Vladimir Kanovei ${ }^{\dagger} \quad$ Vassily Lyubetsky ${ }^{\ddagger}$
}

August 13, 2018

\begin{abstract}
We present a model of set theory, in which, for a given $n \geq 2$, there exists a non-ROD-uniformizable planar $\Pi_{n}^{1}$ set, whose all vertical cross-sections are countable sets (and in fact Vitali classes), while all planar $\boldsymbol{\Sigma}_{n}^{1}$ sets with countable vertical cross-sections are $\boldsymbol{\Delta}_{n+1}^{1}$-uniformizable. Thus it is true in this model, that the ROD-uniformization principle for sets with countable cross-sections fails for a given projective level but holds on all lower levels.
\end{abstract}

\section{Contents}

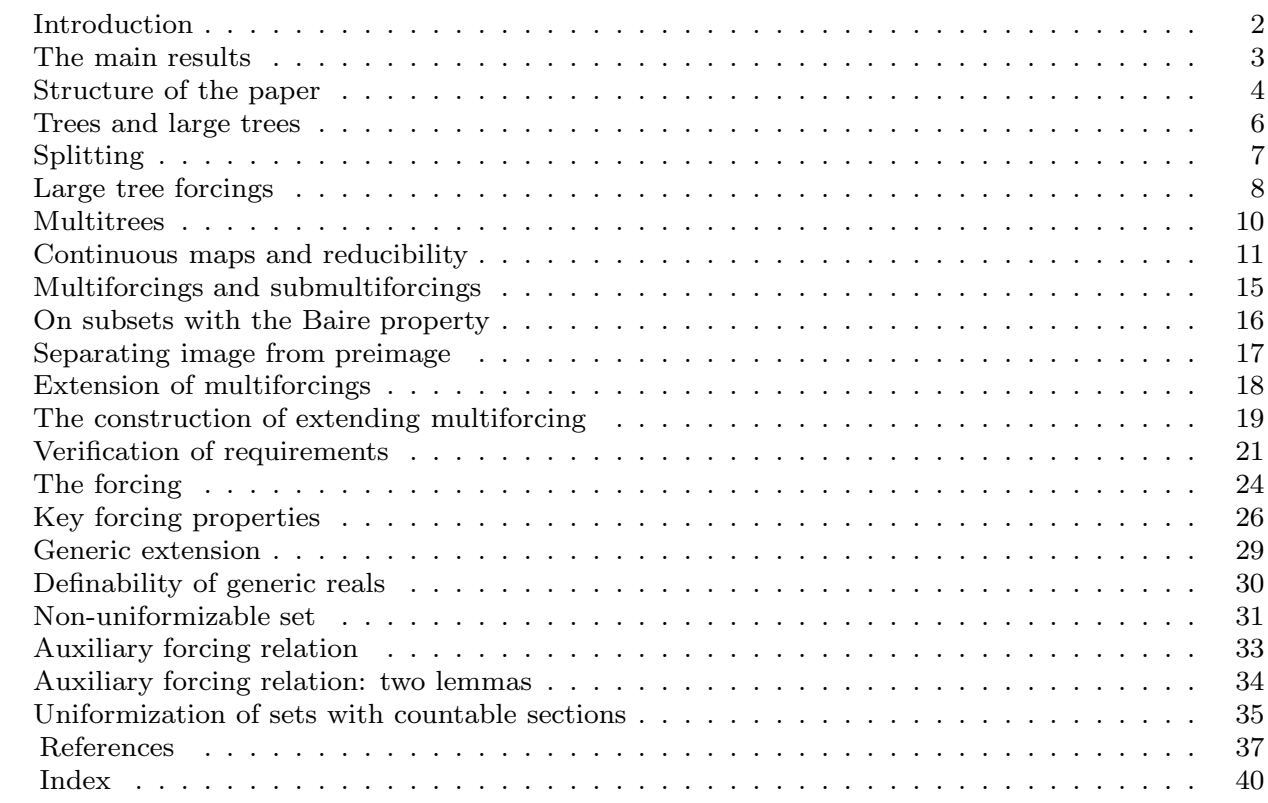

*Vladimir Kanovei's work was supported in part by RFBR grant 17-01-00705. Vladimir Kanovei is also grateful to the Ervin Schroedinger Institute for their support during his visit in December 2016. Vassily Lyubetsky's work was supported in part by RSF grant 14-50-00150.

${ }^{\dagger}$ IITP RAS and RTU (MIIT), kanovei@googlemail.com - contact author.

${ }^{\ddagger}$ IITP RAS and Moscow University, lyubetsk@iitp.ru 


\section{Introduction}

The uniformization problem was introduced into descriptive set theory by Luzin in a short note [29] and in a more detailed paper [30]. ${ }^{1}$ A planar set $Q$ of the real number plane $\mathbb{R} \times \mathbb{R}$ is called uniform (or single-valued), if it intersects every vertical straight line in at most one point. If $Q \subseteq P \subseteq \mathbb{R} \times \mathbb{R}$, the set $Q$ is uniform, and its projection onto the first axis is equal to the same projection of the set $P$, then by Luzin the set $Q$ uniformizes the set $P$. Saying it differently, to uniformize a given planar set $P$ means to choose a point $q_{x}$ in every non-empty vertical cross-section $P_{x}$ of $P$, and then gather all chosen points $q_{x}$, or more precizely, all pairs of the form $\left\langle x, q_{x}\right\rangle$, in a common uniformizing set $Q \subseteq P$. By Luzin, the uniformization problem consists in the question is it possible or not to define a point set $E$ for which we cannot name any uniformizing set $E^{\prime}$. (The translation is quoted from [36, p. 120], the italic text by Luzin and Uspensky.)

In modern set theoretic terminology, there exist exact definitions for such notions of the "naïve" set theory as 'to define', 'to name', 'to give an effective construction', and the like. The largest class of effectively defined sets is the class ROD of real-ordinal definable sets. It consists of all sets definable by a formula with real numbers and ordinals as parameters of the definition. The class ROD contains the subclass OD of all ordinal-definable sets, that is, sets definable by a formula with ordinals (but not reals) as parameters.

There are more special subclasses of ROD and OD, that is, projective classes $\boldsymbol{\Sigma}_{n}^{1}, \boldsymbol{\Pi}_{n}^{1}$, and $\boldsymbol{\Delta}_{n}^{1}=\boldsymbol{\Sigma}_{n}^{1} \cap \boldsymbol{\Pi}_{n}^{1}$ and resp. effective projective classes $\Sigma_{n}^{1}, \Pi_{n}^{1}$, and $\Delta_{n}^{1}=\Sigma_{n}^{1} \cap \Pi_{n}^{1}$; here $n \geq 1$. See [33] in detail, as well as [18], [20], [21], [17], [25], on projective hierarchy. Recall that $\boldsymbol{\Delta}_{1}^{1}=$ Borel sets, $\boldsymbol{\Sigma}_{1}^{1}=$ Suslin, or A-sets, $\boldsymbol{\Pi}_{1}^{1}=$ co-Suslin, or CA-sets, at the level $n=1$.

The following is considered as the most important uniformization theorem in classical descriptive set theory.

Theorem 1.1 (Novikov - Kondo - Addison). If $P$ is a planar set in one of the classes $\Pi_{1}^{1}, \Pi_{1}^{1}, \boldsymbol{\Sigma}_{2}^{1}, \Sigma_{2}^{1}$, then it can be uniformized by a set in the same class.

The key ingredient here was the method of effective choice of a point in a nonempty $\boldsymbol{\Pi}_{1}^{1}$ set by P. S. Novikov, introduced in [31]. On the base of this method, Kondo [27] obtained the result for $\boldsymbol{\Pi}_{1}^{1}$. Addison $[2,1]$ transfered it to the effective class $\Pi_{1}^{1}$. The results for classes $\boldsymbol{\Sigma}_{2}^{1}, \Sigma_{2}^{1}$ are obtained by an elementary argument. On these and other theorems on uniformization and related questions see

\footnotetext{
${ }^{1}$ These notes were not included in Volume II of Luzin's Collected works [32]. However its main elements were considered, partially translated, and analyzed in detail by V. A. Uspensky in [36]. Luzin provides in [29] a quote from a Hadamard's letter in the well-known "Five letters" [9], which can be understood so that Hadamard makes distinction between the pure Zermelo-style choice and choice of elements in non-empty sets by means of a concrete effectively defined function. This gave Luzin an occasion to connect the uniformization problem with the name of Hadamard in the titles of papers $[29,30]$. Uspensky argues in [36, Section 4] that the role of Hadamard is definitely exaggerated here, while the priority with regard to the uniformization problem and related notions belongs to Luzin himself.
} 
references above, as well as $[37,34,10,4,7,6]$ with respect to modern studies, and also in the introductory section of our paper [16].

As for $\Pi_{2}^{1}$ and higher projective classes, similar uniformization theorems are not available since there exist models of set theory in which this or another $\boldsymbol{\Pi}_{2}^{1}$ set $P$ is not uniformizable not only by a projective (of any class) set, but in general by a ROD set. The first such a model was defined by Levy in [28, Theorem 3], where the counter-example required is a pnanar $\Pi_{2}^{1}$ set $P=\left\{\langle x, y\rangle \in \mathbb{R}^{2}: y \notin \mathbf{L}[x]\right\}$, which is not uniformizable by a ROD set in the model. Recall that $\mathbf{L}[x]$ contains all sets Gödel constructible relative to $x$.

Note that every vertical cross-section $P_{x}=\mathbb{R} \backslash \mathbf{L}[x]$ of the set $P$ is either empty (provided $\mathbb{R} \subseteq \mathbf{L}[x]$ ), or else uncountable set, so that it can never non-empty finite or countable. (Moreover, all cross-sections $P_{x}$ can be even co-countable, as e.g. in the Solovay model [35].) The problem of the existence of non-uniformizable $\boldsymbol{\Pi}_{2}^{1}$ sets with countable vertical cross-sections was solved in [14] by a model containing such a set. Then a more precise result was obtained:

Theorem 1.2 (proved in [16], equal to the case $n=2$ in the next Theorem 2.1). There exists a model of $\mathbf{Z F C}$, in which it is true that there is a planar $\Pi_{2}^{1}$ set $W \subseteq \mathbb{R}^{2}$ whose all non-empty vertical cross-sections $W_{x}$ are Vitali classes ${ }^{2}$, which is not uniformizable by a ROD set.

The proof involves a generic extension of the constructible universe $\mathbf{L}$ by a forcing defined as an uncountable product of an invariant version of the Jensen minimal forcing [12]. (See also 28A in [11] on the Jensen forcing.) Some other results obtained by this method include a countable $\Pi_{2}^{1}$ set containing no definable elements [22], a Vitali class with the same properties [13], and an $\Pi_{2}^{1}$ Groszek Laver pair of Vitali classes. See $[16,2.6]$ on the interest in Vitali classes in the context of these results.

\section{The main results}

In continuation of this research line, we prove here the following theorem.

Theorem 2.1. Let $\mathrm{n} \geq 3$. There is a model of the $\mathbf{Z F C}$ set theory, in which the following is true:

(i) there exists a $\Pi_{\mathfrak{m}}^{1}$ set $P \subseteq \mathbb{R} \times \mathbb{R}$, such that all cross-sections $P_{x}=\{y$ : $\langle x, y\rangle \in P\}$ are Vitali classes, and $P$ is not uniformizable by a ROD set;

(ii) if $p \in \mathbb{R}$ then every $\Sigma_{\mathfrak{m}}^{1}(p)$ set $P^{\prime} \subseteq \mathbb{R} \times \mathbb{R}$, whose all vertical cross-sections are countable sets, is uniformizable by a $\Delta_{\mathrm{m}+1}^{1}(p)$ set, hence, by a ROD set.

Following the modern style in descriptive set theory based on certain technical advantages, we shall consider the Cantor discontinuum $2^{\omega}$ with a special equiva-

\footnotetext{
${ }^{2}$ A Vitali class in $\mathbb{R}$ is any set of the form $x+\mathbb{Q}$, that is, a shift of the set $\mathbb{Q}$ of rationals.
} 
lence relation ${ }^{3} E_{0}$, instead of the real line $\mathbb{R}$ with the Vitali equivalence relation, in the substantial part of the proof. Thus the following theorem will be proved:

Theorem 2.2. Let $\mathrm{n} \geq 3$. There is a model of $\mathbf{Z F C}$ in which the following holds:

(i) there exists a $\Pi_{\mathrm{m}}^{1}$ set $W \subseteq 2^{\omega} \times 2^{\omega}$, such that all cross-sections $W_{x}=\{y$ : $\langle x, y\rangle \in W\}$ are $\mathrm{E}_{0}$-classes, and $W$ is not uniformizable by a ROD set;

(ii) if $p \in \mathbb{R}$ then every $\Sigma_{\mathrm{m}}^{1}(p)$ set $W^{\prime} \subseteq 2^{\omega} \times 2^{\omega}$, whose all sections $W_{x}^{\prime}=\{y$ : $\left.\langle x, y\rangle \in W^{\prime}\right\}$ are countable sets, is uniformizable by a $\Delta_{\mathfrak{m}+1}^{1}(p)$ set.

Theorem 2.2 implies Theorem 2.1. The transformation of a set $W$ as in 2.2(i) into a set $P$ as in 2.1(i) is carried out by means of elementary topological arguments, close to a similar transformation in $[16, \S 17]$, so we skip this argument. The derivation of 2.1(ii) from 2.2(ii) is carried out by means of an effective homeomorphism between $\mathbb{R}$ and the co-countable set $X=\left\{x \in 2^{\omega}: \forall m \exists j \geq m(x(j)=0)\right\}$.

\section{Structure of the paper}

The proof of Theorem 2.2 is organized as follows.

The notions related to perfect trees in the set of all dyadic strings $2^{<\omega}$, are introduced in sections 4,5. We consider a collection $\mathbf{L T}$ of all large trees essentially those ones, the relation $\mathrm{E}_{0}$ on which does not admit a Borel transversal. Every set $P \subseteq \mathbf{L T}$ closed under truncating trees at strings, and $\mathrm{E}_{0}$-invariant, i.e., invariant relative to that action of finite strings which induces the relation $E_{0}$ (Remark 4.1), is considered (Section 6) as a forcing notion adding a $P$-generic real $x \in 2^{\omega}$. In fact, as $P$ is $\mathrm{E}_{0}$-invariant, an entire $\mathrm{E}_{0}$-equivalence class $[x]_{\mathrm{E}_{0}}=$ $\left\{y \in 2^{\omega}: x \mathrm{E}_{0} y\right\}$ of generic reals is adjoined.

Then in Section 7 we define the set MT of all multitrees, equal to the countablesupport product $\mathbf{L} \mathbf{T}^{\omega_{1}}$. We study multitrees (including the behaviour of continuous functions on multitrees) in Sections 8-11.

Arguing in the constructible universe $\mathbf{L}$, we define a forcing notion for Theorem 2.2 in Section 15 as the countable-support product $\square=\prod_{\xi<\omega_{1}} \mathbb{P}(\xi) \subseteq \mathbf{M T}$, where each factor $\mathbb{P}(\xi) \subseteq \mathbf{L T}$ has the form of a union $\mathbb{P}(\xi)=\bigcup_{\xi \leq \alpha<\omega_{1}} \mathbb{P}_{\alpha}(\xi)$, where all summands are countable $\mathrm{E}_{0}$-invariant sets $\mathbb{P}_{\alpha}(\xi) \subseteq \mathbf{L T}$ in $\mathbf{L}$, pre-dense in $\mathbb{P}(\xi)$. П-generic extensions of $\mathbf{L}$ will be models for Theorem 2.2. It turns out that each factor $\mathbb{P}(\xi)$ adjoins a $\mathbb{P}(\xi)$-generic real $x_{\xi}$, so that the whole extension is equal to $\mathbf{L}\left[\left\langle x_{\xi}\right\rangle_{\xi<\omega_{1}}\right]$. The following is the first key property of the forcing notion $\square$ :

(1) if $\xi<\omega_{1}$ then the set $\mathbb{P}(\xi)$ is $\mathrm{E}_{0}$-invariant.

The next principal issue in the construction of forcing notions $\mathbb{P}(\xi)$ is similar to the construction of Jensen's forcing in [12] and in some other cases. It consists

\footnotetext{
${ }^{3}$ The relation $\mathrm{E}_{0}$ is defined on $2^{\omega}$ so that $x \mathrm{E}_{0} y$ iff the equality $x(n)=y(n)$ holds for all but finite indices $n$. If $X, Y \subseteq 2^{\omega}$ then $X \equiv_{\mathrm{E}_{0}} Y$ means that every element $a \in X$ is $\mathrm{E}_{0}$ equivalent to some $b \in Y$, and vice versa. See more on this in [19, 20, 25] or elsewhere.
} 
in the definition of every successive "level" $\mathbb{P}_{\alpha}(\xi)$ as generic in some sense over the "levels" $\mathbb{P}_{\gamma}(\xi), \gamma<\alpha$, already defined. This involves a fairly complex construction in Sections 12 - 14, based on the splitting technique for perfest trees. This implies the cardinal preservation (Lemma 16.3), continuous reading of names (Lemma 17.4), as well as the following:

(2) for every index $\xi<\omega_{1}$, the set of all $\mathbb{P}(\xi)$-generic reals in the extension is equal to the $\mathrm{E}_{0}$-class $\left[x_{\xi}\right]_{\mathrm{E}_{0}}$ of the generic real $x_{\xi}$, and also is equal to the intersection $Y_{\xi}=\bigcap_{\xi \leq \alpha<\omega_{1}} \bigcup_{T \in \mathbb{P}_{\alpha}(\xi)}[T]$.

Basically we need here only the equality $\left[x_{\xi}\right]_{\mathrm{E}_{0}}=Y_{\xi}$ (Theorem 18.1). The transformation from a single generic real, as in Jensen, to a $E_{0}$-class of generic reals is implied here by the $\mathrm{E}_{0}$-invariance property as in (1). As a corollary, the definability of the set $W=\left\{\langle\xi, y\rangle: \xi<\omega_{1} \wedge y \in\left[x_{\xi}\right]_{\mathrm{E}_{0}}\right\}$ (the base for a counter-example for $2.2(\mathrm{i}))$ in a $\mathbb{\square}$-generic extension follows from the definability of the indexed set $\left\langle\mathbb{P}_{\alpha}(\xi)\right\rangle_{\xi \leq \alpha<\omega_{1}}$ in $\mathbf{L}$ (Section 19).

Following this idea, we proved Theorem 1.2 in [16] (= case $n=2$ in Theorem 2.2). By the way, the ROD-non-uniformizability of $W$ follows from the $\mathrm{E}_{0^{-}}$ invariance of each component of the forcing notion $\square$ by (1), both in [16] and here.

The main case $m \geq 3$ in Theorem 2.2 differs in that it is necessary to prove claim (ii) in the extension, that immediately holds for $n=2$ by Theorem 1.1 . We

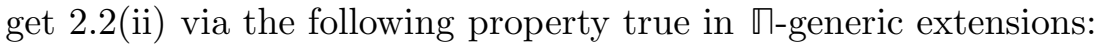

(3) if $x \in 2^{\omega}$ and $X \subseteq 2^{\omega}$ is a countable $\Sigma_{m}^{1}(x)$ set then $X \subseteq \mathbf{L}[x]$.

This property holds in Cohen and some other generic extensions even for $\operatorname{OD}(x)$ sets $X$, see [15]. It also holds in MT-generic extensions of $\mathbf{L}$, where it is implied by the permutation invariance of the forcing notion $\mathbf{M T}=\mathbf{L T}^{\omega_{1}}$ and by a very special feature of those extensions, namely,

(4) if $x, y \in 2^{\omega}$ in a MT-generic extension $\mathbf{L}\left[\left\langle x_{\xi}\right\rangle_{\xi<\omega_{1}}\right]$, and $y \notin \mathbf{L}[x]$, then there exists an ordinal $\xi$ such that $x_{\xi} \in \mathbf{L}[y]$ but $x \in \mathbf{L}\left[\left\langle x_{\eta}\right\rangle_{\eta \neq \xi}\right]$

(compare to [24, Theorem 20] for the $\omega_{1}$-product of the Sacks forcing). П-generic extensions satisfy (4) as well. (Theorem 17.5, based on the study of continuous functions defined on multitrees in Section 8.) Yet this does not directly imply (3) since the forcing notion $\square=\prod_{\xi} \mathbb{P}(\xi)$ is not permutation-invariant as the components $\mathbb{P}(\xi)$ are pairwise different.

This leads to the following modification of the forcing construction. Generally, the construction of $\mathbb{\square}$ can be viewed as the choice of a maximal chain in a certain partially ordered set $\mathscr{P}$ of cardinality $\aleph_{1}$ in $\mathbf{L}$.

(5) We require that this maximal chain intersects all sets dense in $\mathscr{P}$ which belong to the definability class $\Sigma_{m-1}^{1}$. (Theorem 15.4, item (ii) of which contains a property more flexible than this straightforward genericity, but also more difficult for direct formulation.) 
Theorem 15.4 evaluates the definability of this construction. This leads to the definability class $\Pi_{\mathrm{m}}^{1}$ of the set $W$ (see above) in suitable generic extensions. In addition, the forcing notion $\square$ turns out to be enough "generic" in MT, so that it intersects all sets of definability class $\boldsymbol{\Sigma}_{\mathfrak{m}-1}^{1}$, dense in MT (Lemma 16.4). This implies a degree of "similarity" of \-generic and permutation-invariant MTgeneric extensions, up to the m-th level of the projective hierarchy. And further, by fairly complicated arguments in Sections 20-22 (which also make use of (4)), we

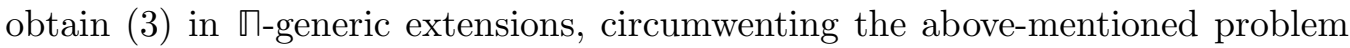
of the permutation noninvariance of $\mathbb{\square}$ and leading to item (ii) of Theorem 2.2.

\section{Trees and large trees}

Here and in the next section, we reproduce, in brief form, some definitions and results from [8] related to perfect and large trees and their transformations.

Strings. $2^{<\omega}$ is the set of all strings (finite sequences) of numbers 0,1 , including the empty string $\Lambda$. If $t \in 2^{<\omega}$ and $i=0,1$ then $t^{\wedge} i$ is the extension of $t$ by $i$ as the rightmost term. If $s, t \in 2^{<\omega}$ then $s \subseteq t$ means that the string $t$ extends $s$ (including the case $s=t$ ), while $s \subset t$ means proper extension. The length of $s$ is $\operatorname{lh}(s)$, and $2^{n}=\left\{s \in 2^{<\omega}: \operatorname{lh}(s)=n\right\}$ (strings of length $n$ ).

Action. Every string $s \in 2^{<\omega}$ acts on $2^{\omega}$ so that if $x \in 2^{\omega}$ then $(s \cdot x)(k)=$ $x(k)+s(k)(\bmod 2)$ for $k<\ln (s)$, and $(s \cdot x)(k)=x(k)$ otherwise. If $X \subseteq 2^{\omega}$ and $s \in 2^{<\omega}$ then let $s \cdot X=\{s \cdot x: x \in X\}$.

Remark 4.1. This action of strings on $2^{\omega}$ induces the relation $\mathrm{E}_{0}$ (footnote 3 ), so that if $x, y \in 2^{\omega}$ then $x \mathrm{E}_{0} y$ is equivalent to $y=s \cdot x$ for a string $s \in 2^{<\omega}$.

Similarly if $s \in 2^{m}, t \in 2^{n}, m \leq n$ then define a string $s \cdot t \in 2^{n}$ so that $(s \cdot t)(k)=t(k)+s(k)(\bmod 2)$ for $k<m$, and $(s \cdot t)(k)=t(k)$ for $m \leq k<n$. But if $m>n$ then let $s \cdot t=(s\lceil n) \cdot t$. In both cases, $\operatorname{lh}(s \cdot t)=\operatorname{lh}(t)$.

If $T \subseteq 2^{<\omega}$ then we let $s \cdot T=\{s \cdot t: t \in T\}$.

Trees. A set $T \subseteq 2^{<\omega}$ is a tree, if for any strings $s \subset t$ in $2^{<\omega}, t \in T$ implies $s \in T$. If $T \subseteq 2^{<\omega}$ is a tree and $u \in T$, then define a trunkated subtree $T \uparrow_{u}=\{t \in T: u \subseteq t \vee t \subseteq u\}$ of $T$. Clearly if $\sigma \in 2^{<\omega}$ then $\sigma \cdot\left(T \uparrow_{u}\right)=(\sigma \cdot T) \uparrow_{\sigma \cdot u}$.

A non-empty tree $T \subseteq 2^{<\omega}$ is perfect, in symbol $T \in \mathbf{P T}$, if it has no endnodes and no isolated branches. In this case, there is a longest string $s=\operatorname{stem}(T) \in T$ satisfying $T=T \uparrow_{s}$ (the stem of $T$ ); then $s^{\wedge} 0 \in T$ and $s^{\wedge} 1 \in T$. If $T \in \mathbf{P T}$ then the set $[T]=\left\{a \in 2^{\omega}: \forall n(a\lceil n \in T)\}\right.$ of all branches of $T$ is a perfect set in $2^{\omega}$.

Large trees. A tree $T \in \mathbf{P T}$ is large, $T \in \mathbf{L T}$, if there exists a system of strings $q_{k}^{i}=q_{k}^{i}[T] \in 2^{<\omega}, k<\omega$ and $i=0,1$, such that

(1) $\ln \left(q_{k}^{0}\right)=\operatorname{lh}\left(q_{k}^{1}\right) \geq 1$ and $q_{k}^{0}(0)=0, q_{k}^{1}(0)=1$ for all $k$;

(2) $T$ consists of all strings of the form $s=r^{\frown} q_{0}^{i_{0}} \frown q_{1}^{i_{1}} \frown q_{2}^{i_{2}} \frown \ldots \frown q_{n}^{i_{n}}$ and their substrings, where $n<\omega, r=\operatorname{stem}(T), i_{k}=0,1$ for all $k$. 
It this case, the set $[T]$ consists of all infinite strings of the form $a=r^{\frown} q_{0}^{i_{0}} \frown q_{1}^{i_{1}} \frown q_{2}^{i_{2}} \frown \ldots \frown q_{n}^{i_{n}} \frown \ldots \in 2^{\omega}$, where $i_{k}=0,1, \forall k$. We let

$$
\operatorname{spl}_{n}(T)=\operatorname{lh}(r)+\operatorname{lh}\left(q_{0}^{i_{0}}\right)+\ln \left(q_{1}^{i_{1}}\right)+\cdots+\operatorname{lh}\left(q_{n-1}^{i_{n-1}}\right)
$$

(independent of the values of $i_{k}=0,1$ ). In particular, $\operatorname{spl}_{0}(T)=\ln (r)$. Thus $\operatorname{spl}(T)=\left\{\operatorname{spl}_{n}(T): n<\omega\right\} \subseteq \omega$ is the set of all splitting levels of $T$.

Remark 4.2. If $T \in \mathbf{L T}$ then the set $[T]$ is $\mathrm{E}_{0}$-nonsmooth, that is, there is no Borel map $f:[T] \rightarrow 2^{\omega}$ satisfying $x \mathrm{E}_{0} y \Longleftrightarrow f(x)=f(y)$ for all $x, y \in[T]$. Conversely, every $\mathrm{E}_{0}$-nonsmooth Borel set $X \subseteq 2^{\omega}$ contains a subset of the form $[T]$, where $T \in \mathbf{L T}$. See [5], [26, 7.1], [25, 10.9] on this category of sets.

We also note that Silver trees are exactly those trees $T \in \mathbf{L T}$ satisfying $q_{k}^{0}[T](j)=q_{k}^{1}[T](j)$ for all $k$ and $1 \leq j<\operatorname{lh}\left(q_{k}^{0}[T]\right)=\operatorname{lh}\left(q_{k}^{1}[T]\right)$.

\section{$5 \quad$ Splitting}

The simple splitting of a tree $T \in \mathbf{L T}$ consists of subtrees $T(\rightarrow i)=T \uparrow_{r}{ }^{\circ}$, $i=0,1$, where $r=\operatorname{stem}(T)$, so that $[T(\rightarrow i)]=\{x \in[T]: x(\ln (r))=i\}$. Then $T(\rightarrow i) \in \mathbf{L T}, \operatorname{stem}(T(\rightarrow i))=r^{\frown} q_{0}^{i}(T), q_{k}^{j}(T(\rightarrow i))=q_{k+1}^{j}(T)$ for all $k$ and $j=0,1$, and $\operatorname{spl}(T(\rightarrow i))=\operatorname{spl}(T) \backslash\left\{\operatorname{spl}_{0}(T)\right\}$.

Splittings can be iterated. We let $T(\rightarrow \Lambda)=T$ for the empty string $\Lambda$, and if $s \in 2^{n}, s \neq \Lambda$ then we define

$$
T(\rightarrow s)=T(\rightarrow s(0))(\rightarrow s(1))(\rightarrow s(2)) \ldots(\rightarrow s(n-1)) \in \mathbf{L T} .
$$

Example 5.1. If $s \in 2^{<\omega}$ then the tree $T[s]=\left\{t \in 2^{<\omega}: s \subseteq t \vee t \subset s\right\}$ belongs to $\mathbf{L T}, \operatorname{stem}(T[s])=s$, and $q_{k}^{i}(T[s])=\langle i\rangle$. In particular $T[\Lambda]=2^{<\omega}$ and $T[s]=$ $\left(2^{<\omega}\right)(\rightarrow s)=\left(2^{<\omega}\right) \uparrow_{s}$ for all $s$.

Lemma 5.2. Let $T \in \mathbf{L T}$. If $s \in 2^{<\omega}$ then $T(\rightarrow s)=T \uparrow_{u[s]}$, where $u[s]=$ $u[s, T]=\operatorname{stem}(T(\rightarrow s))=\operatorname{stem}(T)^{\frown} q_{0}^{s(0)} \frown q_{1}^{s(1)} \frown \ldots \frown q_{n-1}^{s(n-1)} \in T$. Conversely if $u \in T$ then there is a string $s=s[u] \in 2^{<\omega}$ such that $T \uparrow_{u}=T(\rightarrow s)$.

Proof. To prove the converse, we put $s(k)=u\left(\operatorname{spl}_{k}(T)\right)$ for all $k$ such that $\operatorname{spl}_{k}(T)<\operatorname{lh}(u)$.

Lemma 5.3. Let $R \in \mathbf{L T}, n<\omega, h=\operatorname{spl}_{n}(T)$. Then:

(i) if $u, v \in R \cap 2^{h}$ then $T \uparrow_{u}=(u \cdot v) \cdot\left(T \uparrow_{v}\right)$;

(ii) if $s, t \in 2^{n}$ then $R(\rightarrow s)=\sigma \cdot(R(\rightarrow t))$, where $\sigma=u[s, R] \cdot u[t, R]$;

(iii) if $u, v \in R \cap 2^{j}, j<\omega$, then $T \uparrow_{u}=\sigma \cdot\left(T \uparrow_{v}\right)$ for some $\sigma \in 2^{<\omega}$.

Proof. To prove (ii) use Lemma 5.2. To prove (iii) take the least number $h \in$ $\operatorname{spl}(T)$ with $j \leq h$. There is a unique pair of strings $u^{\prime}, v^{\prime} \in 2^{h}$ satisfying $u \subseteq u^{\prime}$, $v \subseteq v^{\prime}$. Then $T \uparrow_{u}=T \uparrow_{u^{\prime}}, T \uparrow_{v}=T \uparrow_{v^{\prime}}$, and $T \uparrow_{u^{\prime}}=\left(u^{\prime} \cdot v^{\prime}\right) \cdot\left(T \uparrow_{v^{\prime}}\right)$. 
Refinement. If $R, T \in \mathbf{L T}$ and $n \in \omega$ then define $R \subseteq_{n} T$ (refinement), if $R(\rightarrow s) \subseteq T(\rightarrow s)$ for all $s \in 2^{n} ; R \subseteq 0 T$ is equivalent to $R \subseteq T$. Clearly $R \subseteq_{n+1} T$ implies $R \subseteq_{n} T$ (and $R \subseteq T$ ). Moreover, if $n \geq 1$ then $R \subseteq_{n} T$ is equivalent to $\operatorname{stem}(R)=\operatorname{stem}(T), q_{k}^{i}[R]=q_{k}^{i}[T]$ for all $i=0,1$ and $k<n-1$, and $q_{n-1}^{i}[T] \subseteq q_{n-1}^{i}[R]$ for all $i=0,1$.

Lemma 5.4. If $T \in \mathbf{L T}, s_{0} \in 2^{n}$, and $U \in \mathbf{L T}, U \subseteq T\left(\rightarrow s_{0}\right)$, then there is a unique $T^{\prime} \in \mathbf{L T}$ satisfying $T^{\prime} \subseteq_{n} T$ and $T^{\prime}\left(\rightarrow s_{0}\right)=U$. We have then

(i) $T^{\prime}(\rightarrow s)=u\left[s_{0}, T\right] \cdot u[s, T] \cdot T^{\prime}\left(\rightarrow s_{0}\right)$ for all $s \in 2^{n}$;

(ii) if $[U]$ is clopen in $\left[T\left(\rightarrow s_{0}\right)\right]$ then $\left[T^{\prime}\right]$ is clopen in $[T]$.

Proof. If $s \in 2^{n}$ then $T(\rightarrow s)=u\left[s_{0}, T\right] \cdot u[s, T] \cdot T\left(\rightarrow s_{0}\right)$ by Lemma 5.3. Put $U_{s}=u\left[s_{0}, T\right] \cdot u[s, T] \cdot U$ for all $s \in 2^{n}$, in particular, $U_{s_{0}}=U$. The tree $T^{\prime}=$ $\bigcup_{u \in 2^{n}} U_{s}$ is as required.

The next lemma is a more complex version of $\subseteq_{n}$-refinement. The proof see Lemma 4.1(iv) in [8].

Lemma 5.5. If $T \in \mathbf{L T}, s_{0}, s_{1} \in 2^{n}$, and $\left.U, V \in \mathbf{L T}, U \subseteq T\left(\rightarrow s_{0}\right\urcorner 0\right), V \subseteq$ $T\left(\rightarrow s_{1} \wedge 1\right.$ ), and $U \equiv_{\mathrm{E}_{0}} V$ (see footnote 3 on $\equiv_{\mathrm{E}_{0}}$ ), then there exists a tree $T^{\prime} \in \mathbf{L T}$ satisfying $T^{\prime} \subseteq_{n+1} T$ and $\left.\left.T^{\prime}\left(\rightarrow s_{0}\right\urcorner 0\right) \subseteq U, T^{\prime}\left(\rightarrow s_{1}\right\urcorner 1\right) \subseteq V$.

Lemma 5.6. Let $\cdots \subseteq_{4} T_{3} \subseteq_{3} T_{2} \subseteq_{2} T_{1} \subseteq_{1} T_{0}$ be an infinite sequence of trees LT. Then $T=\bigcap_{n} T_{n} \in \mathbf{L T}$ and $T \subseteq_{n+1} T_{n}, \forall n$.

Proof. Note that $\operatorname{spl}(T)=\left\{\operatorname{spl}_{n}\left(T_{n}\right): n<\omega\right\}$; this implies both claims.

\section{$6 \quad$ Large tree forcings}

Definition 6.1. Let a $\mathbf{L T}$-forcing be any set $P \subseteq \mathbf{L T}$ satisfying

(A) if $u \in T \in P$ then $T \uparrow_{u} \in P$, or equivalently, if $T \in P$ and $s \in 2^{<\omega}$ then $T(\rightarrow s) \in P$;

(B) $P$ is $\mathrm{E}_{0}$-invariant, i.e., if $T \in P$ and $\sigma \in 2^{<\omega}$ then $\sigma \cdot T \in P$.

If in addition $2^{<\omega} \in P$ then $P$ is a regular $\mathbf{L T}$-forcing.

Any LT-forcing $P$ can be considered as a forcing notion (a set of forcing conditions), ordered so that if $T \subseteq T^{\prime}$ then $T$ is a stronger condition. Such a forcing $P$ adjoins a real $x \in 2^{\omega}$. That is, if a set $G \subseteq P$ is $P$-generic over a ground model $M$, then the intersection $\bigcap_{T \in G}[T]$ contains a unique real $x=x[G] \in 2^{\omega}$, and this real satisfies $M[G]=M[x[G]]$ and $G=\{T \in P: x \in[T]\}$. Reals $x[G]$ of this form are called P-generic. 
Example 6.2. The set LT of all large trees is clearly a LT-forcing. Another example of a LT-forcing is the countable set $P_{\text {coh }}=\left\{T[s]: s \in 2^{<\omega}\right\}$ of all trees $T[s]$ of Example 5.1, i.e. Cohen's forcing. Finally if $\varnothing \neq Q \subseteq \mathbf{L T}$ then

$$
P=\left\{\sigma \cdot\left(T \uparrow_{u}\right): u \in T \in Q \wedge \sigma \in 2^{<\omega}\right\}=\left\{\sigma \cdot(T(\rightarrow s)): T \in Q \wedge s, \sigma \in 2^{<\omega}\right\}
$$

is a $\mathbf{L T}$-forcing by Lemma 5.4 in [16].

A tree $T \in \mathbf{L T}$ is an $n$-collage over a $\mathbf{L T}$-forcing $P$, if we have $T(\rightarrow u) \in P$ for all $u \in 2^{n}$. Thus a 0 -collage is just a tree in $P$, and every $n$-collage is an $n+1$ collage as well.

Lemma 6.3. If $T \in \mathbf{L T}, P$ is a LT-forcing, $u \in 2^{n}$, and $T(\rightarrow u) \in P$, then $T$ is a n-collage over $P$. In particular, under the conditions of Lemma 5.4, if $U \in P$ then the tree $T^{\prime}$ obtained is a $n$-collage over $P$.

Proof. If $v \in 2^{n}$ then $T(\rightarrow v)=\tau \cdot T(\rightarrow u)$ for a string $\tau \in 2^{<\omega}$ by Lemma 5.3. Thus $T(\rightarrow v) \in P$ since $T(\rightarrow u) \in P$.

If $T \in \mathbf{L T}$ and $D \subseteq \mathbf{L T}$ then $X \subseteq \subseteq^{\text {fin }} \cup D$ means that there is a finite set $D^{\prime} \subseteq D$ satisfying $T \subseteq \bigcup D^{\prime}$, or equivalently, $[T] \subseteq \bigcup_{S \in D^{\prime}}[S]$.

Definition 6.4 (extensions). Let $P, Q \subseteq \mathbf{L T}$ be LT-forcings. The forcing $Q$ is an extension of $P$, in symbol $P \sqsubset Q$, if

(1) $Q$ is dense in $P \cup Q$ : if $T \in P$ then $\exists S \in Q(S \subseteq T)$;

(2) If $S \in Q$ then $S \subseteq$ fin $\bigcup P$.

If $\mathfrak{M}$ is any set, and, in addition to $P \sqsubset Q, S \subseteq$ fin $\bigcup D$ holds for all $S \in Q$ and all sets $D \in \mathfrak{M}, D \subseteq P$, pre-dense in $P$, then define $P \sqsubset_{\mathfrak{M}} Q$, $\mathfrak{M}$-extension.

Lemma 6.5. (i) If $Q \subseteq Q^{\prime}$ and $S \subseteq$ fin $\bigcup Q$ for all $S \in Q^{\prime}$ then $Q \sqsubset Q^{\prime}$;

(ii) if $P \sqsubset_{\mathfrak{M}} Q \sqsubset R$ (the second relation is $\sqsubset$, not $\sqsubset_{\mathfrak{M}}$ !) then $P \sqsubset_{\mathfrak{M}} R$;

(iii) if $\left\langle P_{\alpha}\right\rangle_{\alpha<\lambda}$ is an $\sqsubset$-increasing sequence of $\mathbf{L T}$-forcings and $0 \leq \mu<\lambda$ then the set $P_{\mu}$ is pre-dense ${ }^{4}$ in $P=\bigcup_{\alpha<\lambda} P_{\alpha}$.

Proof. (ii) $P \sqsubset R$ is clear. Assume that a set $D \in \mathfrak{M}, D \subseteq P$ is pre-dense in $P$, and $S \in R$. Then $S \subseteq{ }^{\text {fin }} \cup Q$ (since $Q \sqsubset R$ ), thus $S \subseteq T_{1} \cup \cdots \cup T_{n}$, where $T_{1}, \ldots, T_{n} \in Q$. Now $T_{i} \subseteq$ fin $\bigcup D, i=1, \ldots, n$, since $P \sqsubset_{\mathfrak{M}} Q$. It follows that $S \subseteq$ fin $\cup D$ holds as well.

(iii) Let $S \in P_{\alpha}$. If $\alpha \leq \mu$ then $T \in P_{\mu}, T \subseteq S$ holds by 6.4(1). If $\mu<\alpha$ then $S \subseteq T_{1} \cup \cdots \cup T_{n}$, where $T_{1}, \ldots, T_{n} \in P_{\mu}$. Then $S \uparrow_{t} \subseteq T_{i}$ for some $t \in S$ and $i$. But $S^{\prime}=S \uparrow_{t} \in P_{\alpha}$.

\footnotetext{
${ }^{4}$ Pre-density means that every tree $T \in P$ is compatible in $P$ with some $S \in D$, i.e. there is a tree $R \in P$ satisfying $R \subseteq T$ and $R \subseteq S$.
} 


\section{Multitrees}

Let a multitree be any function $\mathbf{T}:|\mathbf{T}| \rightarrow \mathbf{L T}$, where $|\mathbf{T}|=\operatorname{dom} \mathbf{T} \subseteq \omega_{1}$ is at most countable and every value $\mathbf{T}(\xi), \xi \in|\mathbf{T}|$, is a tree in $\mathbf{L T}$. Let MT denote the set of all multitrees. If $\mathbf{T} \in \mathbf{M T}$ then we define a brick in $2^{|\mathbf{T}|}$,

$$
\begin{aligned}
{[\mathbf{T}] } & =\left\{x \in 2^{|\mathbf{T}|}: \forall \xi \in|\mathbf{T}|(x(\xi) \in[\mathbf{T}(\xi)])\right\} \quad= \\
& =\left\{x \in 2^{|\mathbf{T}|}: \forall \xi \forall m(x(\xi)\lceil m \in \mathbf{T}(\xi))\},\right.
\end{aligned}
$$

naturally identified with the cartesian product $\prod_{\xi \in|\mathbf{T}|}[\mathbf{T}(\xi)]$.

If $B \subseteq \omega_{1}$ is at most countable then let $\mathbf{M T}_{B}=\{\mathbf{T} \in \mathbf{M T}:|\mathbf{T}|=B\}$.

The set $\mathbf{M T}$ is ordered componentwise: $\mathbf{T} \leqslant \mathbf{S}$ ( $\mathbf{T}$ is a stronger multitree) whenever $|\mathbf{S}| \subseteq|\mathbf{T}|$ and $\mathbf{T}(\xi) \subseteq \mathbf{S}(\xi)$ for all $\xi \in|\mathbf{S}|$. Thus the ordering of multitrees corresponds to the componentwise inclusion. The weakest (the largest in the sense of $\leqslant$ ) condition in MT is the empty multitree $\boldsymbol{\Lambda}$, satisfying $|\boldsymbol{\Lambda}|=\varnothing$.

It takes some effort to get right versions of definitions and results of Section 5 in the context of multitrees.

Definition 7.1. If $\mathbf{T} \in \mathbf{M T}_{B}$ and $C \subseteq B$, then $\mathbf{T} \uparrow C \in \mathbf{M T}_{C}$ is the ordinary restriction. But if $B \subseteq C$ then a multitree $\mathbf{T} \uparrow C \in \mathbf{M T}_{C}$ is defined by $(\mathbf{T} \uparrow C)(\xi)=$ $\mathbf{T}(\xi)$ for $\xi \in B$, and $(\mathbf{T} \uparrow C)(\xi)=2^{<\omega}$ for $\xi \in C \backslash B$.

Definition 7.2. If $\mathbf{U}$ is a multitree and $\mathbf{D}$ is a set of multitrees, then $\mathbf{U} \subseteq$ fin $\bigvee \mathbf{D}$ means that there is a finite set $\mathbf{D}^{\prime} \subseteq \mathbf{D}$ such that 1) $|\mathbf{V}| \subseteq C=|\mathbf{U}|$ for all $\mathbf{V} \in \mathbf{D}^{\prime}$, and 2) $[\mathbf{U}] \subseteq \bigcup_{\mathbf{V} \in \mathbf{D}^{\prime}}[\mathbf{V} \uparrow C]$ (see Definition 7.1 on $\uparrow$ ). If in addition 3) $[\mathbf{V} \uparrow C] \cap\left[\mathbf{V}^{\prime} \uparrow C\right]=\varnothing$ for all $\mathbf{V} \neq \mathbf{V}^{\prime}$ in $\mathbf{D}^{\prime}$, then we define $\mathbf{U} \subseteq{ }^{\text {fd }} \bigvee \mathbf{D}$.

Definition 7.3. Let $B \subseteq \omega_{1}$ be finite or countable. Fix a function $\phi: \omega \stackrel{\text { onto }}{\longrightarrow} B$ that takes each value infinitely many times, so that if $\xi \in B$ then the set

$$
\phi^{-1}(\xi)=\{k: \phi(k)=\xi\}=\left\{\mathbf{k}_{0 \xi}<\mathbf{k}_{1 \xi}<\mathbf{k}_{2 \xi}<\ldots<\mathbf{k}_{l \xi}<\ldots\right\}
$$

is infinite. Such a function will be called $B$-complete. If $m<\omega$ then let $\boldsymbol{\nu}_{m \xi}$ be equal to the number of indices $k<m, k \in \phi^{-1}(\xi)$. Then $\sum_{\xi \in B} \boldsymbol{\nu}_{m \xi}=m$, and $\boldsymbol{\nu}_{m \xi}>0$ holds for all $\xi \in \phi " m=\{\phi(k): k<m\}$.

Let $m<\omega$ and $\sigma \in 2^{m}$. If $\xi \in \phi " m$ then the set $\phi^{-1}(\xi)$ cuts a substring $\sigma\left\lceil\xi \in 2^{\boldsymbol{\nu}_{m \xi}}\right.$ of length $\ln \left(\sigma\lceil\xi)=\boldsymbol{\nu}_{m \xi}\right.$ off $\sigma$, defined by $\sigma\left\lceil\xi(j)=\sigma\left(\mathbf{k}_{j \xi}\right)\right.$ for all $j<\boldsymbol{\nu}_{m \xi}$. Thus the string $\sigma \in 2^{m}$ splits in a system of strings $\sigma\left[\xi \in 2^{\boldsymbol{\nu}_{m \xi}}\right.$ $(\xi \in \phi " m)$ of total length $\sum_{\xi \in \phi " m} \boldsymbol{\nu}_{m \xi}=m$.

If $\mathbf{T} \in \mathbf{M} \mathbf{T}_{B}$ then define $\mathbf{T}(\Rightarrow \sigma) \in \mathbf{M} \mathbf{T}_{B}$ so that $\mathbf{T}(\Rightarrow \sigma)(\xi)=\mathbf{T}(\xi)(\rightarrow \sigma\lceil\xi)$ for all $\xi \in B$. In particular, if $\xi \in B \backslash \phi " m$ then $\mathbf{T}(\Rightarrow \sigma)(\xi)=\mathbf{T}(\xi)$, where $m=\operatorname{lh}(\sigma)$, because $\ln \left(\sigma\lceil\xi)=\boldsymbol{\nu}_{m \xi}=0\right.$ holds provided $\xi \notin \phi " m$.

If $m<\omega$ and $\sigma, \tau \in 2^{m}$ then define $D[\sigma, \tau]=B \backslash\{\phi(i): i<m \wedge \sigma(i) \neq \tau(i)\}$.

Let $\mathbf{T}, \mathbf{S} \in \mathbf{M T}_{B}$. Define $\mathbf{T} \leqslant_{m} \mathbf{S}$, if $\mathbf{T}(\xi) \subseteq \boldsymbol{\nu}_{m \xi} \mathbf{S}(\xi)$ for all $\xi \in B$. This is equivalent to $\mathbf{T}(\Rightarrow \sigma) \subseteq \mathbf{S}(\Rightarrow \sigma)$ for all $\sigma \in 2^{n}$. 
Lemma 7.4. Under the conditions of Definition 7.3, let $\mathbf{T} \in \mathbf{M T}_{B}$. Then:

(i) if $\sigma \in 2^{<\omega}$ then $\mathbf{T}(\Rightarrow \sigma) \in \mathbf{M T}_{B}$ and the set $[\mathbf{T}(\Rightarrow \sigma)]$ is clopen in $[\mathbf{T}]$;

(ii) if $m<\omega$ and $\sigma, \tau \in 2^{m}$ then $\mathbf{T}(\Rightarrow \sigma)\lceil D[\sigma, \tau]=\mathbf{T}(\Rightarrow \tau)\lceil D[\sigma, \tau]$;

(iii) if $x \in[\mathbf{T}]$, and $U$ is an open nbhd of $x$, then there exists a string $\sigma \in 2^{m}$ satisfying $x \in[\mathbf{T}(\Rightarrow \sigma)] \subseteq U$;

(iv) if $m<\omega, \sigma \in 2^{m}$, and $\mathbf{U} \in \mathbf{M T}_{B}, \mathbf{U} \leqslant \mathbf{T}(\Rightarrow \sigma)$, then there exists a unique multitree $\mathbf{S} \in \mathbf{M T}_{B}$ such that $\mathbf{S} \leqslant_{m} \mathbf{T}$ and $\mathbf{S}(\Rightarrow \sigma)=\mathbf{U}$, and then if $[\mathbf{U}]$ is clopen in $\mathbf{T}(\Rightarrow \sigma)$ then $\mathbf{S}$ is clopen in $\mathbf{T}$;

(v) if $\mathbf{D}$ is a set of multitrees and $\mathbf{T} \subseteq{ }^{\text {fin }} \bigvee \mathbf{D}$, then there is a string $\sigma \in 2^{<\omega}$ and a multitree $\mathbf{S} \in \mathbf{D}$ such that $\mathbf{T}(\Rightarrow \sigma) \leqslant \mathbf{S}$.

Proof. (i) is clear. (iii) We have $\{x\}=\bigcap_{m}[\mathbf{T}(\Rightarrow a \uparrow m)]$ for a suitable sequence $a \in 2^{\omega}$. By the compactness, there is $m$ such that $\mathbf{T}(\Rightarrow a \uparrow m) \subseteq U$.

(iv) If $\xi \in B$ then $\mathbf{U}(\xi) \subseteq \mathbf{T}(\Rightarrow \sigma)(\xi)=\mathbf{T}(\xi)(\rightarrow s)$, where $s=\sigma[\xi$. By Lemma 5.4 there is a tree $S_{\xi} \in \mathbf{L T}$ satisfying $S_{\xi} \subseteq_{n} \mathbf{T}(\xi)$, where $n=\boldsymbol{\nu}_{m \xi}=\operatorname{lh}(s)$, and $S_{\xi}(\rightarrow s)=\mathbf{U}(\xi)$. Let $\mathbf{S}(\xi)=S_{\xi}, \forall \xi$.

(v) There is a multitree $\mathbf{S} \in \mathbf{D}$ such that $|\mathbf{S}| \subseteq B=|\mathbf{T}|$ and the intersection $U=[\mathbf{T}] \cap[\mathbf{S} \uparrow B]$ has a non-empty interior in $[\mathbf{T}]$. It remains to refer to (iii).

Lemma 7.5. Under the conditions of Definition 7.3, let $\ldots \leqslant_{5} \mathbf{T}_{4} \leqslant{ }_{4} \mathbf{T}_{3} \leqslant 3$ $\mathbf{T}_{2} \leqslant{ }_{2} \mathbf{T}_{1} \leqslant{ }_{1} \mathbf{T}_{0}$ be a sequence of multitrees in $\mathbf{M T}_{B}$. Then the multitree $\mathbf{T}=$ $\bigwedge_{n} \mathbf{T}_{n}$, defined by $\mathbf{T}(\xi)=\bigcap_{n} \mathbf{T}_{n}(\xi)$ for all $\xi \in B$, belongs to $\mathbf{M T}_{B}$ and $\mathbf{T} \leqslant{ }_{n+1}$ $\mathbf{T}_{n}$ for all $n$.

Proof. Apply Lemma 5.6 componentwise.

\section{Continuous maps and reducibility}

We consider here some details related to continuous maps defined on bricks emerged from multitrees, similar to some results obtained in $[23,24]$ in the context of perfect sets and trees.

Let $B \subseteq \omega_{1}$ be countable, $\mathbf{T} \in \mathbf{M T}_{B}$, maps $f, g:[\mathbf{T}] \rightarrow \omega^{\omega}$ are continuous.

- $f$ is reduced to $C \subseteq B$ on $[\mathbf{T}]$, if $f(x)=f(y)$ holds whenever $x, y \in[\mathbf{T}]$ and $x\lceil C=y\lceil C$.

- $f$ is reduced to $g$ on $[\mathbf{T}]$, if $f(x)=f(y)$ holds whenever $x, y \in[\mathbf{T}]$ and $g(x)=g(y)$.

- $f$ captures $\alpha \in B$ on $[\mathbf{T}]$, if the co-ordinate map $c_{\alpha}(x)=x(\alpha)$ is reduced to $f$, so that $x(\alpha)=y(\alpha)$ holds whenever $x, y \in[\mathbf{T}]$ and $f(x)=f(y)$.

Lemma 8.1. If $\mathbf{T} \in \mathbf{M T}, C_{0}, C_{1}, \ldots \subseteq B=|\mathbf{T}|, f:[\mathbf{T}] \rightarrow \omega^{\omega}$ is continuous and reduced to every $C_{k}$ on $[\mathbf{T}]$, then $f$ is reduced to $\bigcap_{k} C_{k}$ on $[\mathbf{T}]$. 
Proof. For just two sets, if $C=C_{0} \cap C_{1}$ and $x, y \in[\mathbf{T}], x\lceil C=y\lceil C$, then, using the product structure, find a point $z \in[\mathbf{T}]$ with $z\left\lceil C_{0}=x\left\lceil C_{0}\right.\right.$ and $z\left\lceil C_{1}=y\left\lceil C_{1}\right.\right.$. Then $f(x)=f(z)=f(y)$. The case of finitely many sets follows by simple induction. As for the general case, we can assume that $C_{0} \supseteq C_{1} \supseteq C_{2} \supseteq \ldots$, by the above. Let $C=\bigcap_{k} C_{k}, x, y \in[\mathbf{T}], x\lceil C=y\lceil C$. There is a sequence of points $x_{k} \in[\mathbf{T}]$ satisfying $x_{k}\left\lceil C_{k}=x\left\lceil C_{k}\right.\right.$ and $x_{k} \uparrow\left(B \backslash C_{k}\right)=y \uparrow\left(B \backslash C_{k}\right)$. Then immediately $f\left(x_{k}\right)=f(x), \forall k$. On the other hand, clearly $x_{k} \rightarrow y$, hence, $f\left(x_{k}\right) \rightarrow f(y)$ as $f$ is continuous. Thus $f(x)=f(y)$.

Theorem 8.2. Let $\mathbf{T} \in \mathbf{M T}_{B}, B \subseteq \omega_{1}$ is at most countable, and $f, g:[\mathbf{T}] \rightarrow \omega^{\omega}$ continuous. Then there is a multitree $\mathbf{S} \in \mathbf{M T}_{B}, \mathbf{S} \leqslant \mathbf{T}$, satisfying either (i) $f$ is reduced to $g$ on $[\mathbf{S}]$, or (ii) there is an ordinal $\eta \in B$ such that $f$ captures $\eta$ on $[\mathbf{S}]$ while $g$ is reduced to $B \backslash\{\eta\}$ on $[\mathbf{S}]$.

The co-ordinate map $c_{\eta}(x)=x(\eta)$ is obviously not reducible to $B \backslash\{\eta\}$. Thus the theorem essentially says that the nonreducibility of $f$ to $g$ is detected via co-ordinate maps.

Proof. We argue in terms of Definition 7.3. The plan is to define a sequence of multitrees as in Lemma 7.5, with some extra properties. Let $m<\omega$. A multitree $\mathbf{R} \in \mathbf{M} \mathbf{T}_{B}$ is $m$-good, if $\mathbf{R} \leqslant \mathbf{T}$ and in addition

(1) $f$ if $\sigma \in 2^{m}$ and $\alpha=\phi(m)$ then either $f$ is reduced to $B \backslash\{\alpha\}$ on $[\mathbf{R}(\Rightarrow \sigma)]$, or there is no multitree $\mathbf{R}^{\prime} \in \mathbf{M T}_{B}, \mathbf{R}^{\prime} \leqslant \mathbf{R}(\Rightarrow \sigma)$, such that $f$ is reduced to $B \backslash\{\alpha\}$ on $\left[\mathbf{R}^{\prime}\right]$;

(1) $g$ the same for $g$;

(2) $f$ if $\sigma, \tau \in 2^{m}$, then either (i) $f$ is reduced to $D[\sigma, \tau]=B \backslash\{\phi(i): i<m \wedge$ $\sigma(i) \neq \tau(i)\}$ on $[\mathbf{R}(\Rightarrow \sigma)] \cup[\mathbf{R}(\Rightarrow \tau)]$, or (ii) $f^{\prime \prime}[\mathbf{R}(\Rightarrow \sigma)] \cap f^{\prime \prime}[\mathbf{R}(\Rightarrow \tau)]=\varnothing$;

(2) $g$ the same for $g$.

Lemma 8.3. Under the conditions of the theorem, if $m<\omega$ and a multitree $\mathbf{R} \in \mathbf{M T}_{B}, \mathbf{R} \leqslant \mathbf{T}$, is $m$-good, then there is an $m+1$-good multitree $\mathbf{Q} \in \mathbf{M T}_{B}$, $\mathbf{Q} \leqslant m+1 \mathbf{R}$.

Proof (Lemma). Consider a string $\sigma^{\prime} \in 2^{m+1}$, and first define a multitree $\mathbf{S} \in$ $\mathbf{M T}_{B}, \mathbf{S} \leqslant_{m+1} \mathbf{R}$, satisfying (1) $f$ relatively to this string only. Let $\alpha=\phi(m+1)$. If there exists a multitree $\mathbf{R}^{\prime} \in \mathbf{M T}_{B}, \mathbf{R}^{\prime} \leqslant \mathbf{R}\left(\Rightarrow \sigma^{\prime}\right)$, such that $f$ is reduced to $B \backslash\{\alpha\}$ on $\left[\mathbf{R}^{\prime}\right]$ then let $\mathbf{U}$ be one. If there is no such $\mathbf{R}^{\prime}$ then sipmly put $\mathbf{U}=\mathbf{R}\left(\Rightarrow \sigma^{\prime}\right)$. By Lemma 7.4(iv), there is a multitree $\mathbf{S} \in \mathbf{M T}_{B}$ such that $\mathbf{S} \leqslant{ }_{m+1} \mathbf{R}$ and $\mathbf{S}\left(\Rightarrow \sigma^{\prime}\right)=\mathbf{U}$. Thus the multitree $\mathbf{S}$ satisfies (1) $f$ with respect to $\sigma^{\prime}$. Now take $\mathbf{S}$ as the "new" multitree $\mathbf{R}$, consider another string $\sigma^{\prime} \in 2^{m+1}$, and do the same as above. Consider all strings $2^{m+1}$ consecutively, with the same procedure. In the end, this yields a multitree $\mathbf{S} \in \mathbf{M T}_{B}, \mathbf{S} \leqslant_{m+1} \mathbf{R}$, satisfying (1) $f$ for all strings in $2^{m+1}$. 
Now take care of $(2) f$. Let $\sigma^{\prime}, \tau^{\prime} \in 2^{m+1}$. Note that if $\sigma^{\prime}(m)=\tau^{\prime}(m)$ then $D\left[\sigma^{\prime}, \tau^{\prime}\right]=D\left[\sigma^{\prime} \uparrow m, \tau^{\prime}\lceil m]\right.$, so that $(2) f$ relatively to $\sigma^{\prime}, \tau^{\prime}$ follows from $(2) f$ relatively to $\sigma^{\prime} \uparrow m, \tau^{\prime} \uparrow m$. Thus it suffices to consider only pairs in $2^{m+1}$ of the form $\sigma^{\frown} 0, \tau^{\urcorner} 1$, where $\sigma, \tau \in 2^{m}$. Consider one such a pair $\sigma^{\prime}=\sigma^{\frown} 0, \tau^{\prime}=\tau^{\frown} 1$, and define a multitree $\mathbf{Q} \in \mathbf{M T}_{B}, \mathbf{Q} \leqslant{ }_{m+1} \mathbf{S}$, satisfying $(2) f$ relatively to this pair. The sets $C^{\prime}=D\left[\sigma^{\prime}, \tau^{\prime}\right]$ and $C=D[\sigma, \tau]$ satisfy $C^{\prime}=C \backslash\left\{\eta_{0}\right\}$, where $\eta_{0}=\phi(m)$, while multitrees $\mathbf{S}\left(\Rightarrow \sigma^{\prime}\right), \mathbf{S}\left(\Rightarrow \tau^{\prime}\right)$ satisfy $\mathbf{S}\left(\Rightarrow \sigma^{\prime}\right)\left\lceil C^{\prime}=\mathbf{S}\left(\Rightarrow \tau^{\prime}\right)\left\lceil C^{\prime}\right.\right.$.

However by $(2) f$ for the pair $\sigma, \tau$, either $f$ is reduced to $C$ on $[\mathbf{S}(\Rightarrow \sigma)] \cup$ $[\mathbf{S}(\Rightarrow \tau)]$, or $f^{\prime \prime}[\mathbf{S}(\Rightarrow \sigma)] \cap f^{\prime \prime}[\mathbf{S}(\Rightarrow \tau)]=\varnothing$. In the second case, immediately $f^{\prime \prime}\left[\mathbf{S}\left(\Rightarrow \sigma^{\prime}\right)\right] \cap f^{\prime \prime}\left[\mathbf{S}\left(\Rightarrow \tau^{\prime}\right)\right]=\varnothing$. Thus we can wlog assume that $f$ is reduced to $C$ on $[\mathbf{S}(\Rightarrow \sigma)] \cup[\mathbf{S}(\Rightarrow \tau)]$.

If now $f$ is reduced to $B^{\prime}=B \backslash\left\{\eta_{0}\right\}$ on $\left[\mathbf{S}\left(\Rightarrow \sigma^{\prime}\right)\right] \cup\left[\mathbf{S}\left(\Rightarrow \tau^{\prime}\right)\right]$, then $f$ is reduced to $C^{\prime}=C \cap B^{\prime}$ as well on $\left[\mathbf{S}\left(\Rightarrow \sigma^{\prime}\right)\right] \cup\left[\mathbf{S}\left(\Rightarrow \tau^{\prime}\right)\right]$ by Lemma 8.1, as required.

Thus suppose that $f$ is not reduced to $B^{\prime}$ on $\left[\mathbf{S}\left(\Rightarrow \sigma^{\prime}\right)\right] \cup\left[\mathbf{S}\left(\Rightarrow \tau^{\prime}\right)\right]$. Then there are points $x_{0} \in\left[\mathbf{S}\left(\Rightarrow \sigma^{\prime}\right)\right], y_{0} \in\left[\mathbf{S}\left(\Rightarrow \tau^{\prime}\right)\right]$ with $x_{0}\left\lceil B^{\prime}=y_{0}\left\lceil B^{\prime}\right.\right.$ and $f\left(x_{0}\right) \neq f\left(y_{0}\right)$, i.e., $f\left(x_{0}\right)(k)=p \neq q=f\left(y_{0}\right)(k)$ for some $k ;\{p, q\}=\{0,1\}$. As $f$ is continuous, there exist relatively open subsets $X \subseteq\left[\mathbf{S}\left(\Rightarrow \sigma^{\prime}\right)\right], Y \subseteq\left[\mathbf{S}\left(\Rightarrow \tau^{\prime}\right)\right]$, such that $x_{0} \in$ $X, y_{0} \in Y, f(x)(k)=p$ and $f(y)(k)=q$ for all $x \in X, y \in Y$. We assume wlog that there exists a finite set $H \subseteq B$ containing $\eta_{0}$, and for each $\eta \in H$ strings $u_{\eta} \in U_{\eta}=\mathbf{S}\left(\Rightarrow \sigma^{\prime}\right)(\eta)=\mathbf{S}(\eta)\left(\rightarrow \sigma^{\prime}\lceil\eta), v_{\eta} \in V_{\eta}=\mathbf{S}\left(\Rightarrow \tau^{\prime}\right)(\eta)=\mathbf{S}(\eta)\left(\rightarrow \tau^{\prime}\lceil\eta)\right.\right.$ of equal length $\ln \left(u_{\eta}\right)=\ln \left(v_{\eta}\right)=\ell_{\eta} \geq \boldsymbol{\nu}_{m+1, \eta}$, such that $\sigma^{\prime}\left\lceil\eta \subseteq u_{\eta}, \tau^{\prime}\left\lceil\eta \subseteq v_{\eta}\right.\right.$,

$X=\left\{x \in[\mathbf{S}]: \forall \eta \in H\left(x(\eta) \in\left[U_{\eta}^{\prime}\right]\right)\right\}$, and $Y=\left\{y \in[\mathbf{S}]: \forall \eta \in H\left(y(\eta) \in\left[V_{\eta}^{\prime}\right]\right)\right\}$,

where $U_{\eta}^{\prime}=U_{\eta} \uparrow u_{\eta}$ and $V_{\eta}^{\prime}=V_{\eta} \uparrow v_{\eta}(\eta \in H)$ are trees in LT. Note that $\sigma^{\prime} \uparrow \eta=\tau^{\prime}\lceil\eta$, $u_{\eta}=v_{\eta}, U_{\eta}=V_{\eta}$, and $U_{\eta}^{\prime}=V_{\eta}^{\prime}$ for all $\eta \in H, \eta \neq \eta_{0}$, as $x_{0}\left\lceil B^{\prime}=y_{0}\left\lceil B^{\prime}\right.\right.$. This allows us to define a required multitree $\mathbf{Q}$ as follows.

If $\eta \in B \backslash H$ then simply put $Q_{\eta}=\mathbf{S}(\eta)$.

Let $\eta \in H, \eta \neq \eta_{0}$. Then $\sigma^{\prime}\left\lceil\eta=\tau^{\prime}\left\lceil\eta\right.\right.$, and this string $s=\sigma^{\prime}\left\lceil\eta=\tau^{\prime}\lceil\eta\right.$ of length $\boldsymbol{\nu}_{m \eta}=\boldsymbol{\nu}_{m+1, \eta}$ satisfies $\mathbf{S}\left(\Rightarrow \sigma^{\prime}\right)(\eta)=\mathbf{S}\left(\Rightarrow \tau^{\prime}\right)(\eta)=\mathbf{S}(\eta)(\rightarrow s)$. The string $u=u_{\eta}=v_{\eta}$ belongs to $\mathbf{S}(\eta)(\rightarrow s)$, so that the subtree $W_{\eta}=\mathbf{S}(\eta)(\rightarrow s) \uparrow_{u}=$ $U_{\eta}^{\prime}=V_{\eta}^{\prime}$ belongs to $\mathbf{L T}$ and $W_{\eta} \subseteq \mathbf{S}(\eta)(\rightarrow s)$. By Lemma 5.4 there exists a tree $Q_{\eta} \in \mathbf{L T}$ satisfying $Q_{\eta} \subseteq_{\boldsymbol{\nu}_{m+1, \eta}} \mathbf{S}(\eta)$ and $Q_{\eta}(\rightarrow s)=W_{\eta}$.

Finally let $\eta=\eta_{0} \in H$. The strings $\sigma^{\prime}\left[\eta_{0} \neq \tau^{\prime}\left\lceil\eta_{0}\right.\right.$ of equal length $\boldsymbol{\nu}_{m+1, \eta_{0}}=$ $\boldsymbol{\nu}_{m \eta_{0}}+1$ are different in this case since $\sigma^{\prime}\left\{\eta_{0}\left(\boldsymbol{\nu}_{m \eta_{0}}\right)=\sigma^{\prime}(m)=0 \neq 1=\tau^{\prime}(m)=\tau^{\prime} \uparrow\right.$ $\eta_{0}\left(\boldsymbol{\nu}_{m \eta_{0}}\right)$. The equalities $\sigma^{\prime}\left\lceil\eta_{0}=\tau^{\prime}\left\lceil\eta_{0}, U_{\eta_{0}}=V_{\eta_{0}}, U_{\eta_{0}}^{\prime}=V_{\eta_{0}}^{\prime}\right.\right.$ generally speaking, fail as well. But still $u_{\eta_{0}} \in U_{\eta_{0}}=\mathbf{S}\left(\eta_{0}\right)\left(\rightarrow \sigma^{\prime}\left\lceil\eta_{0}\right), v_{\eta_{0}} \in V_{\eta_{0}}=\mathbf{S}\left(\eta_{0}\right)\left(\rightarrow \tau^{\prime}\left\lceil\eta_{0}\right)\right.\right.$, $\operatorname{lh}\left(u_{\eta_{0}}\right)=\operatorname{lh}\left(u_{\eta_{0}}\right)=\ell_{\eta_{0}}$, and $U_{\eta_{0}}^{\prime}=U_{\eta_{0}} \uparrow_{u_{\eta_{0}}} \subseteq U_{\eta_{0}}, V_{\eta_{0}}^{\prime}=V_{\eta_{0}} \uparrow_{v_{\eta_{0}}} \subseteq V_{\eta_{0}}$. Thus we have $U_{\eta_{0}}^{\prime} \equiv_{\mathrm{E}_{0}} V_{\eta_{0}}^{\prime}$ by Lemma 5.3. Therefore by Lemma 5.5, there exists a tree $Q_{\eta_{0}} \in \mathbf{L T}$ such that $Q_{\eta_{0}} \subseteq_{\boldsymbol{\nu}_{m+1, \eta_{0}}} \mathbf{S}\left(\eta_{0}\right), Q_{\eta_{0}}\left(\rightarrow \sigma^{\prime}\left\lceil\eta_{0}\right) \subseteq U_{\eta_{0}}^{\prime}\right.$, and $Q_{\eta_{0}}\left(\rightarrow \tau^{\prime}\left[\eta_{0}\right) \subseteq V_{\eta_{0}}^{\prime}\right.$

Thus a tree $Q_{\eta} \in \mathbf{L T}$, satisfying $Q_{\eta} \subseteq \boldsymbol{\nu}_{m+1, \eta} \mathbf{S}(\eta)$, has been defined for all $\eta \in B$ so that if $\eta \in H$ then $Q_{\eta}\left(\rightarrow \sigma^{\prime}[\eta) \subseteq U_{\eta}^{\prime}\right.$ and $Q_{\eta}\left(\rightarrow \tau^{\prime}\lceil\eta) \subseteq V_{\eta}^{\prime}\right.$. This allows us to define a required multitree $\mathbf{Q} \in \mathbf{M T}_{B}$ by $\mathbf{Q}(\eta)=Q_{\eta}$ for all $\eta \in B$. 
Then $\mathbf{Q} \leqslant{ }_{m+1} \mathbf{S}$, and by construction $\mathbf{Q}\left(\Rightarrow \sigma^{\prime}\right) \subseteq X$ and $\mathbf{Q}\left(\Rightarrow \tau^{\prime}\right) \subseteq Y$, so that $f^{\prime \prime}\left[\mathbf{Q}\left(\Rightarrow \sigma^{\prime}\right)\right] \cap f^{\prime \prime}\left[\mathbf{Q}\left(\Rightarrow \tau^{\prime}\right)\right]=\varnothing$.

To conclude, a multitree $\mathbf{Q} \in \mathbf{M T}_{B}, \mathbf{Q} \leqslant_{m+1} \mathbf{S}$, satisfying (2) $f$ relative to the pair $\sigma^{\prime}, \tau^{\prime} \in 2^{m+1}$ considered, is defined. Going over all pairs in $2^{m+1}$ consecutively, we obtain a multitree $\mathbf{Q} \in \mathbf{M} \mathbf{T}_{B}, \mathbf{Q} \leqslant_{m+1} \mathbf{S}$, satisfying $(2) f$ with respect to all pairs $\sigma^{\prime}, \tau^{\prime} \in 2^{m+1}$.

Then proceed with the same procedure for $g$.

(Lemma)

Come back to the proof of the theorem. Lemma 8.3 yields an infinite sequence $\ldots \leqslant 3 \mathbf{S}_{2} \leqslant 2 \mathbf{S}_{1} \leqslant 1 \mathbf{S}_{0}=\mathbf{T}$ of multitrees $\mathbf{S}_{m} \in \mathbf{M} \mathbf{T}_{B}$, such that each $\mathbf{S}_{m}$ is a $m$ good. The limit multitree $\mathbf{S}=\bigwedge_{m} \mathbf{S}_{m} \in \mathbf{M} \mathbf{T}_{B}$ satisfies $\mathbf{S} \leqslant_{m+1} \mathbf{S}_{m}$ for all $m$ by Lemma 7.5. Therefore $\mathbf{S}$ is $m$-good for every $m$, hence we can freely use (1) $f, g$ and (2) $f, g$ in the arguments below.

Case 1: if $m<\omega ; \sigma, \tau \in 2^{m}$; and $f "[\mathbf{S}(\Rightarrow \sigma)] \cap f "[\mathbf{S}(\Rightarrow \tau)]=\varnothing$; then $g "[\mathbf{S}(\Rightarrow \sigma)] \cap g "[\mathbf{S}(\Rightarrow \tau)]=\varnothing$. Prove that $f$ is reduced to $g$ on $[\mathbf{S}]$ in this case, as required by (i) of the theorem. Let $x, y \in[\mathbf{S}]$ and $f(x) \neq f(y)$; show that $g(x) \neq$ $g(y)$. Pick $a, b \in 2^{\omega}$ satisfying $\{x\}=\bigcap_{m}\left[\mathbf{S}(\Rightarrow a\lceil m)]\right.$ and $\{y\}=\bigcap_{m}[\mathbf{S}(\Rightarrow b\lceil m)]$. As $x \neq y$, we have $f "[\mathbf{S}(\Rightarrow a\lceil m)] \cap f "[\mathbf{S}(\Rightarrow b\lceil m)]=\varnothing$ for some $m$ by the continuity and compactness. Then by the Case 1 assumption, $g$ " $[\mathbf{S}(\Rightarrow a\lceil m)] \cap$ $g "[\mathbf{S}(\Rightarrow b\lceil m)]=\varnothing$ holds, hence $g(x) \neq g(y)$.

Case 2: not Case 1, that is, by (2) $g$, there is a pair of strings $\sigma^{\prime}=\sigma^{\wedge} i, \tau^{\prime}=$ $\tau^{\frown} j \in 2^{m+1}, m<\omega$, such that $f "\left[\mathbf{S}\left(\Rightarrow \sigma^{\prime}\right)\right] \cap f^{\prime \prime}\left[\mathbf{S}\left(\Rightarrow \tau^{\prime}\right)\right]=\varnothing$, but $g$ is reduced to $C^{\prime}=D\left[\sigma^{\prime}, \tau^{\prime}\right]$ on $\left[\mathbf{S}\left(\Rightarrow \sigma^{\prime}\right)\right] \cup\left[\mathbf{S}\left(\Rightarrow \tau^{\prime}\right)\right]$. We assume that $m$ is the least possible for this case. We are going to prove that the multitree $\mathbf{S}(\Rightarrow \sigma)$ satisfies (ii) of the theorem with the ordinal $\eta_{0}=\phi(m)$, that is, $\left(^{*}\right) g$ is reduced to $B \backslash\left\{\eta_{0}\right\}$ on $[\mathbf{S}(\Rightarrow \sigma)]$, and $(* *) f$ captures $\eta_{0}=\phi(m)$ on $[\mathbf{S}(\Rightarrow \sigma)]$.

To prove $\left(^{*}\right)$ note that $(\dagger) f$ is reduced to $C=D[\sigma, \tau]$ on $[\mathbf{S}(\Rightarrow \sigma)] \cup[\mathbf{S}(\Rightarrow \tau)]$. Indeed otherwise $f^{\prime \prime}[\mathbf{S}(\Rightarrow \sigma)] \cap f^{\prime \prime}[\mathbf{S}(\Rightarrow \tau)]=\varnothing$ by $(2) f$, hence $g$ is not reduced to $C$ on $[\mathbf{S}(\Rightarrow \sigma)] \cup[\mathbf{S}(\Rightarrow \tau)]$ by the minimality of $m$, thus $g "[\mathbf{S}(\Rightarrow \sigma)] \cap g "[\mathbf{S}(\Rightarrow \tau)]=\varnothing$ by (2) $g$, then obviously $g "\left[\mathbf{S}\left(\Rightarrow \sigma^{\prime}\right)\right] \cap g "\left[\mathbf{S}\left(\Rightarrow \tau^{\prime}\right)\right]=\varnothing$, which contradicts to the assumption that $g$ is reduced to $C^{\prime}$ on $\left[\mathbf{S}\left(\Rightarrow \sigma^{\prime}\right)\right] \cup\left[\mathbf{S}\left(\Rightarrow \tau^{\prime}\right)\right]$ because $\left[\mathbf{S}\left(\Rightarrow \sigma^{\prime}\right)\right]\left\lceil C^{\prime}=\right.$ $\left[\mathbf{S}\left(\Rightarrow \tau^{\prime}\right)\right]\left\lceil C^{\prime}\right.$ by Lemma $7.4($ ii).

Note that $i \neq j$, as otherwise $C=C^{\prime}$, and a contradiction easily follows. Thus let, e.g., $\sigma^{\prime}=\sigma^{\urcorner} 0, \tau^{\prime}=\tau^{\frown} 1$. Then $C^{\prime}=C \backslash\left\{\eta_{0}\right\}$, and the multitree $\mathbf{S}\left(\Rightarrow \sigma^{\prime}\right)$ witnesses that $g$ is reduced to $B \backslash\left\{\eta_{0}\right\}$ on $[\mathbf{S}(\Rightarrow \sigma)]$ by $(1) g$. Thus we have $\left(^{*}\right)$.

We further claim that $(\ddagger) f$ is not reduced to $B \backslash\left\{\eta_{0}\right\}$ on any multitree $\mathbf{U} \in \mathbf{M} \mathbf{T}_{B}, \mathbf{U} \leqslant \mathbf{S}(\Rightarrow \sigma)$. Indeed otherwise $f$ is reduced to $B \backslash\left\{\eta_{0}\right\}$ on $[\mathbf{S}(\Rightarrow \sigma)]$ by (1) $f$. Then $f$ is reduced to $C^{\prime}=C \cap\left(B \backslash\left\{\eta_{0}\right\}\right)$ on $[\mathbf{S}(\Rightarrow \sigma)]$ by Lemma 8.1. It follows that $f$ is reduced to $C^{\prime}$ on the union $W=[\mathbf{S}(\Rightarrow \sigma)] \cup[\mathbf{S}(\Rightarrow \tau)],{ }^{5}$ hence

\footnotetext{
${ }^{5}$ Let $x, y \in W$ and $x\left\lceil C^{\prime}=y\left\lceil C^{\prime}\right.\right.$. As $\mathbf{S}(\Rightarrow \sigma) \uparrow C=\mathbf{S}(\Rightarrow \tau) \uparrow C$ by Lemma 7.4(ii), there are $x^{\prime}, y^{\prime} \in[\mathbf{S}(\Rightarrow \sigma)]$ with $x\left\lceil C=x^{\prime}\left\lceil C\right.\right.$ and $y\left\lceil C=y^{\prime} \uparrow C\right.$. But $f$ is reduced to $C$ on $W$ by ( $\left.\dagger\right)$. Thus $f(x)=f\left(x^{\prime}\right), f(y)=f\left(y^{\prime}\right)$. Finally $f\left(x^{\prime}\right)=f\left(y^{\prime}\right)$ since $f$ is reduced to $C^{\prime}$ on $[\mathbf{S}(\Rightarrow \sigma)]$.
} 
on $W^{\prime}=\left[\mathbf{S}\left(\Rightarrow \sigma^{\prime}\right)\right] \cup\left[\mathbf{S}\left(\Rightarrow \tau^{\prime}\right)\right] \subseteq W$ as well. But this contradicts to $f "\left[\mathbf{S}\left(\Rightarrow \sigma^{\prime}\right)\right] \cap$ $f^{\prime \prime}\left[\mathbf{S}\left(\Rightarrow \tau^{\prime}\right)\right]=\varnothing$, since $\mathbf{S}\left(\Rightarrow \sigma^{\prime}\right)\left\lceil C^{\prime}=\mathbf{S}\left(\Rightarrow \tau^{\prime}\right)\left\lceil C^{\prime}\right.\right.$ by Lemma 7.4(ii). As required.

We now prove $\left({ }^{* *}\right)$, that is, $f$ captures $\eta_{0}$ on $[\mathbf{S}(\Rightarrow \sigma)]$. Let $x, y \in[\mathbf{S}(\Rightarrow \sigma)]$ and $f(x)=f(y)$; prove that $x\left(\eta_{0}\right)=y\left(\eta_{0}\right)$. Indeed $\{x\}=\bigcap_{n}[\mathbf{S}(\Rightarrow a\lceil n)]$ and $\{y\}=\bigcap_{n}\left[\mathbf{S}(\Rightarrow b\lceil n)]\right.$, where $a, b \in 2^{\omega}, \sigma \subset a, \sigma \subset b$. Let $D[a, b]=$ $\bigcap_{n} D[a\lceil n, b\lceil n]$. Then $x\lceil D[a, b]=y\lceil D[a, b]$, since $\mathbf{S}(\Rightarrow a\lceil n)\lceil D[a\lceil n, b\lceil n]=$ $\mathbf{S}\left(\Rightarrow b\lceil n)\left\lceil D\left[a\left\lceil n, b\lceil n]\right.\right.\right.\right.$ for all $n$. Thus it remains to check that $\eta_{0} \in D[a\lceil n, b\lceil n]$ for all $n$.

Suppose towards the contrary that $\eta_{0}=\phi(m) \notin D[a\lceil n, b\lceil n]$ for some $n$. Note that $n>m$ because $a\lceil m=b\lceil m=\sigma$. However $f$ is reduced to $D[a\lceil n, b\lceil n]$ on $\left[\mathbf{S}(\Rightarrow a\lceil n)]\right.$ by $(2) f$, since $f(x)=f(y)$. Yet we have $\eta_{0} \notin D[a\lceil n, b\lceil n]$, therefore, $D\left[a\left\lceil n, b\lceil n] \subseteq B \backslash\left\{\eta_{0}\right\}\right.\right.$. It follows that $f$ is reduced to $B \backslash\left\{\eta_{0}\right\}$ on $[\mathbf{S}(\Rightarrow a\lceil n)]$. But this contradicts to $(\ddagger)$ above with $\mathbf{U}=\mathbf{S}(\Rightarrow a\lceil n)$.

To conclude Case 2, we have checked $(*)$ and $(* *)$, as required.

\section{Multiforcings and submultiforcings}

Let a multiforcing be any function $\mathbf{P}$, such that $|\mathbf{P}|=\operatorname{dom} \mathbf{P} \subseteq \omega_{1}$ and every value $\mathbf{P}(\xi), \xi \in|\mathbf{P}|$, is a $\mathbf{L T}$-forcing. Thus a multiforcing is a partial $\omega_{1}$-sequence of LT-forcings. A multiforcing $\mathbf{P}$ is small, if the base $|\mathbf{P}|$ and each forcing $\mathbf{P}(\xi)$, $\xi \in|\mathbf{P}|$, are at most countable sets, and regular, if $2^{<\omega} \in \mathbf{P}(\xi)$ for all $\xi \in|\mathbf{P}|$.

If $\mathbf{P}$ is a multiforcing then let $\mathbf{M T}(\mathbf{P})$ denote the set of all multitrees $\mathbf{T}$ such that $|\mathbf{T}| \subseteq|\mathbf{P}|$ and $\mathbf{T}(\xi) \in \mathbf{P}(\xi)$ for all $\xi \in|\mathbf{P}|$. The set $\mathbf{M T}(\mathbf{P})$ can be identified with the countable base product $\prod_{\xi \in|\mathbf{P}|} \mathbf{P}(\xi)$.

The next definition introduces a type of sets containing multitrees and satisfying some minimal closure conditions.

Definition 9.1. Let $\mathbf{P}$ be a regular multiforcing. A set $\mathfrak{S} \subseteq \mathbf{M T}(\mathbf{P})$ is a $s u b$ multiforcing, if it satisfies the following:

(I) if $\mathbf{T} \in \mathfrak{S}, \xi \in|\mathbf{T}|$, and $T \in \mathbf{P}(\xi)$, then the multitree $\mathbf{S}$, defined by $|\mathbf{S}|=|\mathbf{T}|$, $\mathbf{S}(\xi)=T$, and $\mathbf{S}(\eta)=\mathbf{T}(\eta)$ for $\eta \neq \xi$, also belongs to $\mathfrak{S} ;$

(II) if $\mathbf{T} \in \mathfrak{S}, \xi \in|\mathbf{P}| \backslash|\mathbf{T}|$, and $T \in \mathbf{P}(\xi)$, then the multitree $\mathbf{S}$, defined by $|\mathbf{S}|=|\mathbf{T}| \cup\{\xi\}, \mathbf{S}(\xi)=T$, and $\mathbf{S} \uparrow|\mathbf{T}|=\mathbf{T}$, also belongs to $\mathbf{S} ;$

(III) if $\mathbf{T}, \mathbf{S} \in \mathcal{S}$ then the multitree $\mathbf{T}^{\prime}=\mathbf{T} \uparrow(|\mathbf{T}| \cup|\mathbf{S}|)$, defined by $\left|\mathbf{T}^{\prime}\right|=$ $|\mathbf{T}| \cup|\mathbf{S}|, \mathbf{T}^{\prime}(\xi)=\mathbf{T}(\xi)$ for $\xi \in|\mathbf{T}|$, and $\mathbf{T}^{\prime}(\xi)=2^{<\omega}$ for $\xi \in|\mathbf{S}| \backslash|\mathbf{T}|$, also belongs to $\mathfrak{S}$.

Example 9.2. Let $\mathbf{P}$ be a regular multiforcing, $B=|\mathbf{P}|$. Then $\mathbf{M T}(\mathbf{P})$ is the largest submultiforcing in $\mathbf{M T}(\mathbf{P})$, while the smallest submultiforcing in $\mathbf{M T}(\mathbf{P})$ is the countable set $\mathfrak{S}_{\text {coh }}^{B}$ of all multitrees $\mathbf{T} \in \mathbf{M T}(\mathbf{P})$ such that $|\mathbf{T}| \subseteq B$ is finite and $\mathbf{T}(\xi) \in P_{\text {coh }}$ (Example 6.2) for all $\xi \in|\mathbf{T}|$, Cohen's forcing in $\left(2^{\omega}\right)^{B}$.

Multitrees $\mathbf{T}, \mathbf{S}$ in a submultiforcing $\mathfrak{S} \subseteq \mathbf{M T}$ are compatible in $\mathfrak{S}$, if there is a multitree $\mathbf{U} \in \mathfrak{S}$ satisfying $\mathbf{U} \leqslant \mathbf{T}$ and $\mathbf{U} \leqslant \mathbf{S}$. A set $\mathbf{D} \subseteq \mathfrak{S}$ is: 
dense in $\mathfrak{S}$, when $\forall \mathbf{T} \in \mathfrak{S} \exists \mathbf{S} \in \mathbf{D}(\mathbf{S} \leqslant \mathbf{T})$;

open dense in $\mathfrak{S}$, if in addition $\forall \mathbf{T}, \mathbf{S} \in \mathfrak{S}(\mathbf{T} \leqslant \mathbf{S} \in \mathbf{D} \Longrightarrow \mathbf{T} \in \mathbf{D})$;

pre-dense in $\mathfrak{S}$, if the set $\mathbf{D}^{+}=\{\mathbf{T} \in \mathfrak{S}: \exists \mathbf{S} \in \mathbf{D}(\mathbf{T} \leqslant \mathbf{S})\}$ is dense in $\mathfrak{S}$.

In the context of Definition 7.3, a multitree $\mathbf{T}$ (not necessarily $\mathbf{T} \in \mathbf{S}$ !) is called an $m$-collage over $\mathfrak{S}$, if $\mathbf{T}(\Rightarrow u) \in \mathfrak{S}$ for all strings $u \in 2^{m}$. Thus a 0 collage is any multitree in $\mathfrak{S}$, while every $m$-collage is an $m+1$-collage as well by the closure properties in Definition 9.1.

Lemma 9.3. Let $\mathbf{P}$ be a multiforcing, $\mathfrak{S} \subseteq \mathbf{M T}(\mathbf{P})$ be a submultiforcing, $\mathbf{T} \in$ $\mathbf{M T}_{B}$. Then, in terms of Definition 7.3, the following holds:

(i) if $\sigma \in 2^{<\omega}$ and $\mathbf{T} \in \mathfrak{S}$ then $\mathbf{T}(\Rightarrow \sigma) \in \mathfrak{S}$;

(ii) if $\sigma \in 2^{n}$ and $\mathbf{T}(\Rightarrow \sigma) \in \mathfrak{S}$, then $\mathbf{T}$ is an $n$-collage over $\mathfrak{S}$;

(iii) if $\mathbf{T}$ is an $m$-collage over $\mathfrak{S}$, and $\mathbf{D} \subseteq \mathfrak{S}$ is clopen in $\mathfrak{S}$, then there is a multitree $\mathbf{S} \in \mathbf{M} \mathbf{T}_{B}$, which is an $m$-collage over $\mathfrak{S}$ and satisfies $\mathbf{S} \leqslant_{m} \mathbf{T}$ and $\mathbf{S}(\Rightarrow \sigma) \in \mathbf{D}$ for all $\sigma \in 2^{m}$;

(iv) if $U \subseteq[\mathbf{T}]$ is a nbhd of $x_{0} \in[\mathbf{T}]$ in $[\mathbf{T}]$ then there is a multitree $\mathbf{S} \in \mathbf{S}$ such that $|\mathbf{S}|=B, x_{0} \in[\mathbf{S}] \subseteq U$, and $\mathbf{S} \leqslant \mathbf{T}$.

Proof. (i) Use property 6.1(A) of LT-forcings with the closure properties of Definition 9.1. Further, splitting the operation $(\Rightarrow \sigma)$ to components as in Definition 7.3, immediately reduces (ii) to Lemma 6.3.

(iii) If $\sigma \in 2^{m}$ then by Lemma 7.4(iv) there exists a multitree $\mathbf{S} \in \mathbf{M T}_{B}$, $\mathbf{S} \leqslant_{m} \mathbf{T}$, satisfying $\mathbf{S}(\Rightarrow \sigma) \in \mathbf{D}$ for this $\sigma$. And $\mathbf{S}$ is still an $m$-collage over $\mathfrak{S}$ by (ii). Iterate this procedure, going over all strings in $\sigma \in 2^{m}$.

(iv) We refer to (i) and Lemma 7.4(iii).

\section{On subsets with the Baire property}

This and the next section present two applications of Lemma 7.5 to the construction of multitrees with certain properties. Comparing to Theorem 8.2, where Lemma 7.5 was also used in the course of the proof, here by necessity we'll have to consider intermediate multitrees related to some multiforcing.

Lemma 10.1. Let $\mathbf{T} \in \mathbf{M T}, B=|\mathbf{T}|$. If the set $X \subseteq[\mathbf{T}]$ has the Baire property inside $[\mathbf{T}]$ then there is a multitree $\mathbf{S} \in \mathbf{M T}_{B}$ such that $[\mathbf{S}] \subseteq X$ or $[\mathbf{S}] \subseteq[\mathbf{T}] \backslash X$.

Proof. Fix a $B$-complete function $\phi: \omega \stackrel{\text { onto }}{\longrightarrow} B$. In our assumptions, $X$ or $[\mathbf{T}] \backslash X$ is co-meager on a non-empty clopen $U \subseteq[\mathbf{T}]$. The cases are symmetric, hence we can assume that $X$ is co-meager on $U$. Note that $[\mathbf{T}(\Rightarrow \sigma)] \subseteq U$ for some $\sigma \in 2^{<\omega}$ by Lemma 7.4(iii). Yet the set $[\mathbf{T}(\Rightarrow \sigma)]$ itself is clopen in $[\mathbf{T}]$, and $X^{\prime}=X \cap[\mathbf{T}(\Rightarrow \sigma)]$ is co-meager in $[\mathbf{T}(\Rightarrow \sigma)]$. Thus the task is reduced to the 
case when the set $X$ is co-meager in $[\mathbf{T}]$, and this will be assumed below. In this assumption, we can further suppose that $X=\bigcap_{n} U_{n}$, where every set $U_{n} \subseteq[\mathbf{T}]$ is topologically open and dense in $[\mathbf{T}]$.

Case 1: there exists a multitree $\mathbf{S} \in \mathbf{M T}_{B}$ such that $\mathbf{S} \leqslant \mathbf{T}$ and $[\mathbf{S}] \cap U_{n}=\varnothing$ for some $n$. Then $[\mathbf{S}] \subseteq[\mathbf{T}] \backslash X$, as required.

Case 2: if $\mathbf{S} \in \mathbf{M T}_{B}$ and $\mathbf{S} \leqslant \mathbf{T}$ then $[\mathbf{S}] \cap U_{n} \neq \varnothing$ for all $n$. Define a regular multiforcing $\mathbf{P}$ such that $|\mathbf{P}|=B$ and if $\xi \in B$ then

$$
\mathbf{P}(\xi)=\left\{s \cdot(\mathbf{T}(\xi)(\rightarrow t)): s \in 2^{<\omega} \wedge t \in \mathbf{T}(\xi)\right\} \cup P_{\text {coh }} \quad \text { (see Definition 6.2) } .
$$

Consider the submultiforcing $\mathfrak{S}=\{\mathbf{S} \in \mathbf{M T}(\mathbf{P}):|\mathbf{T}|=B\} ; \mathbf{T} \in \mathbf{S}$. We claim that for every $m$ the set

$$
\mathbf{D}_{m}=\left\{\mathbf{S} \in \mathbf{S}:[\mathbf{S}] \cap[\mathbf{T}]=\varnothing \text { or } \mathbf{S} \leqslant \mathbf{T} \wedge[\mathbf{S}] \subseteq U_{m}\right\}
$$

is open dense in $\mathfrak{S}$ (in the sense of Section 9). The openness is obvious. To prove the density let $\mathbf{T}^{\prime} \in \mathfrak{S}$. If $\left[\mathbf{T}^{\prime}\right] \nsubseteq[\mathbf{T}]$ then $U=\left[\mathbf{T}^{\prime}\right] \backslash[\mathbf{T}]$ is topologically open in $\left[\mathbf{T}^{\prime}\right]$ and non-empty. By Lemma $7.4($ iii), there exists a multitree $\mathbf{S} \in \mathbf{S}$ such that $[\mathbf{S}] \subseteq U$, i.e., $\mathbf{S} \leqslant \mathbf{T}^{\prime}$ and $\mathbf{S} \in \mathbf{D}_{m}$. Thus assume that $\mathbf{T}^{\prime} \leqslant \mathbf{T}$. Then $\left[\mathbf{T}^{\prime}\right] \cap U_{m} \neq \varnothing$ by the Case 2 assumption. Applying Lemma 7.4(iii), we find a multitree $\mathbf{S} \in \mathfrak{S}$ satisfying $[\mathbf{S}] \subseteq U_{m}$, that is, $\mathbf{S} \in \mathbf{D}_{m}$. The density is proved.

Now Lemma 9.3(iii) implies a sequence $\ldots \leqslant 4 \mathbf{T}_{3} \leqslant{ }_{3} \mathbf{T}_{2} \leqslant{ }_{2} \mathbf{T}_{1} \leqslant 1 \mathbf{T}_{0} \leqslant \mathbf{T}$ of multitrees $\mathbf{T}_{m} \in \mathbf{M T}_{B}$ with $\mathbf{T}_{m}(\Rightarrow \sigma) \in \mathbf{D}_{m}$ for all $m$ and $\sigma \in 2^{m}$. The multitree $\mathbf{S}=\bigwedge_{m} \mathbf{T}_{m}$ (Lemma 7.5) then satisfies $[\mathbf{S}] \subseteq U_{m}, \forall m$, hence $[\mathbf{S}] \subseteq X$.

\section{Separating image from preimage}

If $x_{0} \in X \subseteq 2^{\omega}, f: X \rightarrow 2^{\omega}$ is continuous, and $f\left(x_{0}\right) \neq x_{0}$, then there exists a nbhd $U$ of $x_{0}$ in $X$ whose $f$-image $f$ " $U$ does not intersect $U$. The next theorem is a version of this claim.

Definition 11.1. Let $\mathbf{T} \in \mathbf{M T}_{B}$ and $\xi \in B$. A continuous map $f:[\mathbf{T}] \rightarrow 2^{\omega}$ is called simple on $[\mathbf{T}]$ for $\xi$, if there exists a string $\sigma \in 2^{<\omega}$ such that $f(x)=\sigma \cdot x(\xi)$ holds for all $x \in[\mathbf{T}]$.

Theorem 11.2. Under the conditions of Definition 7.3, let $\xi \in B=|\mathbf{P}|, \mathfrak{S} \subseteq$ $\mathbf{M T}(\mathbf{P})$ is a submultiforcing, $m, n<\omega, \mathbf{T} \in \mathbf{M} \mathbf{T}_{B}$ is an $m$-collage over $\mathfrak{S}$, and $f:[\mathbf{T}] \rightarrow 2^{\omega}$ is continuous. Then:

(i) if $U \in \mathbf{L T}$ is an $n$-collage over a $\mathbf{L T}$-forcing $P$, then there exists a multitree $\mathbf{T}^{\prime} \in \mathbf{M} \mathbf{T}_{B}$ and a tree $U^{\prime} \in \mathbf{L T}$ such that $\mathbf{T}^{\prime} \leqslant_{m} \mathbf{T}, U^{\prime} \subseteq_{n} U, \mathbf{T}^{\prime}$ is an $m$ collage over $\mathfrak{S}, U^{\prime}$ is a $n$-collage over $P$, and $\left[U^{\prime}\right] \cap f^{\prime \prime}\left[\mathbf{T}^{\prime}\right]=\varnothing$;

(ii) if $\xi \in B=|\mathbf{P}|$, and if $r \in 2^{<\omega}$ then $f$ is not simple for $\xi$ on $\mathbf{T}(\Rightarrow r)$, then there is a multitree $\mathbf{T}^{\prime} \in \mathbf{M} \mathbf{T}_{B}$ such that $\mathbf{T}^{\prime} \leqslant_{m} \mathbf{T}, \mathbf{T}^{\prime}$ is an $m$-collage over $\mathfrak{S}$, and $\left[\mathbf{T}^{\prime}(\xi)\right] \cap f^{\prime \prime}\left[\mathbf{T}^{\prime}\right]=\varnothing$. 
Proof. (i) To begin with consider a pair of strings $u \in 2^{m}, s \in 2^{n}$. Let $x_{0} \in$ $[\mathbf{T}(\Rightarrow u)]$. Pick $y_{0} \in[U(\rightarrow s)], y_{0} \neq f\left(x_{0}\right)$. As $f$ is continuous, there exists an open nbhd $G \subseteq[\mathbf{T}]$ of $x_{0}$ in $\mathbf{T}(\Rightarrow u)$ and a string $t \in U(\rightarrow s)$, satisfying $t \subset y_{0}$, and $t \not \subset x(\xi)$ for all $x \in G$. Put $V=U \uparrow_{t}$. Then $V \in P$ and $V \subseteq U(\rightarrow s)$. By Lemma 5.4, there exists a tree $U^{\prime} \in \mathbf{L T}$, such that $U^{\prime} \subseteq_{n} U$ and $U^{\prime}(\rightarrow s)=V$. Note that $U^{\prime}$ is an $n$-collage over $P$ by Lemma 6.3 .

On the other hand, by Lemma 9.3(iv), there is a multitree $\mathbf{S} \in \mathfrak{S}$ such that $|\mathbf{S}|=B$ and $[\mathbf{S}] \subseteq G$. By Lemma 7.4(iv), there is a multitree $\mathbf{T}^{\prime} \in \mathbf{M} \mathbf{T}_{B}$ satisfying $\mathbf{T}^{\prime} \leqslant_{m} \mathbf{T}$ and $\mathbf{T}^{\prime}(\Rightarrow u)=\mathbf{S}$. Note that $\mathbf{T}^{\prime}$ is an $m$-collage over $\mathfrak{S}$ by Lemma 9.3(ii). Thus $\mathbf{T}^{\prime}$ and $U^{\prime}$ witness (i) partially: $\left[U^{\prime}(\rightarrow s)\right] \cap f^{\prime \prime}\left[\mathbf{T}^{\prime}(\Rightarrow u)\right]=\varnothing$ holds, not yet $\left[U^{\prime}\right] \cap f^{\prime \prime}\left[\mathbf{T}^{\prime}\right]=\varnothing$. However this procedure can be iterated, going over all pairs of strings $u \in 2^{m}, s \in 2^{n}$. This leads to the result required.

(ii) As in the first part, it suffices, given a pair of strings $r, s \in 2^{m}$ (possibly $r=$ $s)$, to find an $m$-collage $\mathbf{T}^{\prime} \in \mathbf{M} \mathbf{T}_{B}$ over $\mathfrak{S}$, satisfying $\mathbf{T}^{\prime} \leqslant m$ T and $\left[\mathbf{T}^{\prime}(\Rightarrow s)(\xi)\right] \cap$ $f^{\prime \prime}\left[\mathbf{T}^{\prime}(\Rightarrow r)\right]=\varnothing$. The tree $T=\mathbf{T}(\xi)$ belongs to $\mathbf{P}(\xi) \subseteq \mathbf{L T}$, and $\mathbf{T}(\Rightarrow s)(\xi)=$ $T\left(\rightarrow s^{\prime}\right), \mathbf{T}(\Rightarrow r)(\xi)=T\left(\rightarrow r^{\prime}\right)$, where $s^{\prime}=s\left[\xi, t^{\prime}=t\lceil\xi\right.$ are strings of length $n=\boldsymbol{\nu}_{m \xi}$, see Definition 7.3. Now $T\left(\rightarrow s^{\prime}\right)=\tau \cdot T\left(\rightarrow r^{\prime}\right)$ by Lemma 5.3, where $\tau=u\left[s^{\prime}, T\right] \cdot u\left[r^{\prime}, T\right]$. But $f$ is not simple on $\mathbf{T}(\Rightarrow r)$, hence there exists a point $x_{0} \in \mathbf{T}(\Rightarrow r)$ such that $f\left(x_{0}\right) \neq \tau \cdot x_{0}(\xi)$. We have two strings $v \neq w$ in $2^{<\omega}$ of equal length $\ln (v)=\operatorname{lh}(w)>\operatorname{lh}(\tau)$, satisfying $v \subset f\left(x_{0}\right)$ and $w \subset \tau \cdot x_{0}(\xi)$. We put $w^{\prime}=\tau \cdot w$; then $w^{\prime} \subset x_{0}(\xi)$.

But $f$ is continuous, hence using Lemma 9.3 as above, we find a multitree $\mathbf{S} \in \mathbf{S}$ such that $|\mathbf{S}|=B, \mathbf{S} \leqslant \mathbf{T}(\Rightarrow r)$, and if $x \in[\mathbf{S}]$ then $v \subset f(x), w \subset \tau \cdot x(\xi)$, $w^{\prime} \subset x(\xi)$. And further we find a multitree $\mathbf{T}^{\prime} \in \mathbf{M} \mathbf{T}_{B}$ satisfying $\mathbf{T}^{\prime} \leqslant_{m} \mathbf{T}$ and $\mathbf{T}^{\prime}(\Rightarrow r)=\mathbf{S}$, and being an $m$-collage over $\mathfrak{S}$.

We claim that $\left[\mathbf{T}^{\prime}(\Rightarrow s)(\xi)\right] \cap f^{\prime \prime}\left[\mathbf{T}^{\prime}(\Rightarrow r)\right]=\varnothing$. Indeed by construction if $x \in[\mathbf{S}]=\left[\mathbf{T}^{\prime}(\Rightarrow r)\right]$ thrn $v \subset f(x)$. Thus it remains to check that $w \subset b$ for all $b \in\left[\mathbf{T}^{\prime}(\Rightarrow s)(\xi)\right]$. Note that $\mathbf{T}^{\prime}(\Rightarrow s)(\xi)=T^{\prime}\left(\rightarrow s^{\prime}\right)$ and $\mathbf{T}^{\prime}(\Rightarrow r)(\xi)=T^{\prime}\left(\rightarrow r^{\prime}\right)$, where $T^{\prime}=\mathbf{T}^{\prime}(\xi) \in \mathbf{P}(\xi)$. On the other hand $T^{\prime}$ is a tree in LT and $T^{\prime} \subseteq_{n}$ $T$, hence $T^{\prime}\left(\rightarrow s^{\prime}\right)=\tau \cdot T^{\prime}\left(\rightarrow r^{\prime}\right)$ by Lemma 5.4. Thus if $b \in\left[\mathbf{T}^{\prime}(\Rightarrow s)(\xi)\right]$ then $a=\tau \cdot b \in\left[\mathbf{T}^{\prime}(\Rightarrow r)(\xi)\right]=\left[T^{\prime}\left(\rightarrow r^{\prime}\right)\right]$. It follows that $w^{\prime} \subset a$ by the choice of $\mathbf{S}=\mathbf{T}^{\prime}(\Rightarrow r)$. Then $w \subset b=\tau \cdot a$ (since $\left.w=\tau \cdot w^{\prime}\right)$, as required.

\section{Extension of multiforcings}

The forcing notion for the proof of Theorem 2.1 will be defined as an $\omega_{1}$-union of an increasing $\omega_{1}$-sequence of multiforcings. Definition 12.3 below contains conditions which every step of the construction will have to obey. We begin with the following definition.

Definition 12.1 (coding continuous maps). Let $B \subseteq \omega_{1}$ be at most countable. A code of a continuous map $\left(2^{\omega}\right)^{B} \rightarrow 2^{\omega}$ is an indexed family $\mathbf{c}=\left\langle U_{i}^{\mathbf{c}}(k)\right\rangle_{k<\omega, i=0,1}$ of finite sets $U_{i}^{\mathbf{c}}(k) \subseteq \mathfrak{S}_{\text {coh }}^{B}$ (see Example 9.2), such that for all $k$ :

(1) if $\mathbf{T} \in U_{0}^{\mathbf{c}}(k)$ and $\mathbf{S} \in U_{0}^{\mathbf{c}}(k)$ then $[\mathbf{T} \uparrow B] \cap[\mathbf{S} \uparrow B]=\varnothing$, and 
(2) $\bigcup_{k<\omega, i=0,1} \bigcup_{\mathbf{T} \in U_{i}^{\mathbf{c}}(k)}[\mathbf{T} \uparrow B]=\left(2^{\omega}\right)^{B}$.

Let $\mathrm{CCF}_{B}$ denote the set of all such codes.

We set $\mathrm{CCF}=\bigcup_{B \subseteq \omega_{1}, \operatorname{card} B \leq \aleph_{0}} \mathrm{CCF}_{B}$, and if $\mathbf{c} \in \mathrm{CCF}_{B}$ then $|\mathbf{c}|=B$.

The coded map $f=f^{\mathbf{c}}:\left(2^{\omega}\right)^{B} \rightarrow 2^{\omega}$ itself is defined as follows in this case: $f^{\mathbf{c}}(x)(k)=i$, if there is a multitree $\mathbf{T} \in U_{i}^{\mathbf{c}}(k)$ such that $x \in[U \uparrow B]$. Make use of (1) to show that the definition is sound.

We skip a routine proof of the following lemma, based on the compactness of the spaces considered.

Lemma 12.2. If $B \subseteq \omega_{1}$ is countable, $X \subseteq\left(2^{\omega}\right)^{B}$ closed, and a map $f: X \rightarrow 2^{\omega}$ is continuous, then there is a code $\mathbf{c} \in \mathrm{CCF}_{B}$ such that $f=f^{\mathbf{c}} \uparrow X$.

Definition 12.3 (in $\mathbf{L}$ ). Let $\mathfrak{M}$ be a countable transitive model of theory $\mathbf{Z F C}^{\prime}$, which includes all ZFC axioms except for the power set axiom, but with the axiom which claims the existence of $\mathscr{P}(\omega)$. (This implies the existence of the ordinal $\omega_{1}$ and sets like $2^{\omega}, \mathbf{P T}, \mathbf{L T}$ of cardinality $\mathfrak{c}=2^{\aleph_{0}}$.)

Recall that $\mathbf{L}_{\alpha}$ is $\alpha$-th level of the Gödel constructible hierarchy.

Let $\mathbf{P} \in \mathfrak{M}$ be a regular (small) multiforcing. Then $|\mathbf{P}|=B \in \mathfrak{M}$ and $\alpha=\sup B=\bigcup B<\omega_{1}$. We let $\mathfrak{S}(\mathbf{P})$ denote the closure of $\mathbf{M T}(\mathbf{P}) \cap \mathbf{L}_{\alpha}$ in $\mathbf{M T}(\mathbf{P})$ with respect to the three operations of Definition 9.1. Thus $\mathfrak{S}(\mathbf{P}) \in \mathfrak{M}$, $\mathfrak{S}(\mathbf{P}) \subseteq \mathbf{M T}(\mathbf{P}), \mathfrak{S}(\mathbf{P})$ is a countable submultiforcing.

Note that $\mathfrak{S}(\mathbf{P})$ does not depend on $\mathfrak{M}$.

A multiforcing $\mathbf{Q}$ (not necessarily in $\mathfrak{M}$ ) is an $\mathfrak{M}$-extension of $\mathbf{P}$, in symbol $\mathbf{P} \sqsubset_{\mathfrak{M}} \mathbf{Q}$, if the following holds:

(A) $|\mathbf{Q}|=|\mathbf{P}|$ and $\mathbf{Q}$ is a small multiforcing;

(B) if $\xi \in|\mathbf{P}|$ then $\mathbf{P}(\xi) \sqsubset_{\mathfrak{M}} \mathbf{Q}(\xi)$ in the sense of Definition 6.4;

(C) if $\mathbf{T} \in \mathbf{S}(\mathbf{P})$ then there is a multitree $\mathbf{S} \in \mathbf{M T}(\mathbf{Q})$ satisfying $\mathbf{S} \leqslant \mathbf{T}$ and $\mathbf{S} \subseteq{ }^{\mathrm{fd}} \bigvee \mathbf{D}$ for all open dense sets $\mathbf{D} \subseteq \mathfrak{S}(\mathbf{P}), \mathbf{D} \in \mathfrak{M}$;

(D) if $\mathbf{T} \in \mathbf{S}(\mathbf{P}), \xi \in|\mathbf{T}|$, a map $f:\left(2^{\omega}\right)^{\mid \mathbf{T}} \mid \rightarrow 2^{\omega}$ is continuous and has a code in $\mathrm{CCF}_{|\mathbf{T}|} \cap \mathfrak{M}$, then there exists a multitree $\mathbf{S} \in \mathbf{M T}(\mathbf{Q})$ such that $|\mathbf{S}|=|\mathbf{T}|$, $\mathbf{S} \leqslant \mathbf{T}$, and either (i) there is a string $\sigma \in 2^{<\omega}$ such that $f(x)=\sigma \cdot x(\xi)$ for all $x \in[\mathbf{S}]$, or (ii) $f(x) \notin[U]$ for all $x \in[\mathbf{S}]$ and $U \in \mathbf{Q}(\xi)$.

Theorem 12.4 (in $\mathbf{L}$ ). Let $\mathfrak{M}$ be a countable transitive model of $\mathbf{Z F C}^{\prime}$, and $\mathbf{P} \in \mathfrak{M}$ be a regular (small) multiforcing. Then there is an $\mathfrak{M}$-extension $\mathbf{Q}$ of $\mathbf{P}$.

The proof of the theorem follows in the two next sections. The construction of $\mathbf{Q}$ is presented in Section 13, the proof of its properties follows in Section 14.

\section{The construction of extending multiforcing}

The following definitions formalize construction of generic multitrees for the proof of Theorem 12.4, by means of Lemma 7.5. 
- Arguing under the conditions of Theorem 12.4, we let $B=|\mathbf{P}|$ and $\mathfrak{S}=$ $\mathfrak{S}(\mathbf{P})$, so that $B<\omega_{1}$ and $\mathfrak{S} \subseteq \mathbf{M T}(\mathbf{P})$ is a countable submultiforcing.

- During the course of the proof of Theorem 12.4, i. e., to the end of Section14, we fix a $B$-complete function $\phi: \omega \stackrel{\text { onto }}{\longrightarrow} B$. This allows to use the notation of Definition 7.3.

To begin with, we reduce all multitrees $\mathbf{T} \in \mathfrak{S}$ to the domain $B$, substituting each of them by its copy $\mathbf{T}^{\uparrow}=\mathbf{T} \uparrow B$ (see Definition 7.1). Thus, by the regularity of $\mathbf{P}$, we have $\mathbf{T}^{\uparrow} \in \mathbf{M T}(\mathbf{P})$ and $\left|\mathbf{T}^{\uparrow}\right|=B$, and by definition $\mathbf{T}^{\uparrow}(\xi)=\mathbf{T}(\xi)$ for $\xi \in|\mathbf{T}|$, but $\mathbf{T}^{\uparrow}(\xi)=2^{<\omega}$ for $\xi \in B \backslash|\mathbf{T}|$. We put $\mathfrak{S}^{\uparrow}=\left\{\mathbf{T}^{\uparrow}: \mathbf{T} \in \mathfrak{S}\right\}$, this is a submultiforcing, too.

Definition 13.1. A system (over $\mathfrak{S}^{\uparrow}$ ) is any function $\varphi: \operatorname{dom} \varphi \rightarrow \mathbf{M T}_{B}$ where $\operatorname{dom} \varphi \subseteq \omega \times \omega$ is finite, and if $\langle k, m\rangle \in \operatorname{dom} \varphi$ then

(1) if $n<m$ then $\langle k, n\rangle$ also belongs to $\operatorname{dom} \varphi$;

(2) $\varphi(k, m)$ is a tree in $\mathbf{M T}_{B}$ and a $m$-collage over $\mathfrak{S}^{\uparrow}$, and $|\varphi(k, m)|=B$;

(3) if $m>0$ then $\varphi(k, m) \leqslant m \varphi(k, m-1)$.

In this case, let $\nu_{k}^{\varphi}$ denote the largest number $m$ satisfying $\langle k, m\rangle \in \operatorname{dom} \varphi$, but $\nu_{k}^{\varphi}=-1$ if there is no such $m$. Let $|\varphi|=\left\{k: \nu_{k}^{\varphi} \geq 0\right\}$, a finite set.

Let $\operatorname{Sys}\left(\mathfrak{S}^{\uparrow}\right)$ denote the set of all systems.

A system $\varphi$ extends a system $\psi$, in symbol $\psi \subseteq \varphi$, if $\operatorname{dom} \psi \subseteq \operatorname{dom} \varphi$ and $\psi=\varphi\lceil\operatorname{dom} \psi ;$ while $\psi \subset \varphi$ will denote strict extension.

Lemma 13.2 (elementary). Suppose that $\varphi \in \operatorname{Sys}\left(\mathfrak{S}^{\uparrow}\right)$. Then

(i) if $k \in|\varphi|$ and $m=\nu_{k}^{\varphi}$ then the extension $\varphi^{\prime}$ of system $\varphi$ by $\nu_{k}^{\varphi^{\prime}}=m+1$ and $\varphi^{\prime}(k, m+1)=\varphi^{\prime}(k, m)$ is a system extending $\varphi$;

(ii) if $k \notin|\varphi|$ and $\mathbf{T} \in \mathfrak{S}^{\uparrow}$, then the extension $\varphi^{\prime}$ of system $\varphi$ by $\operatorname{dom} \varphi^{\prime}=$ $\operatorname{dom} \varphi \cup\{\langle k, 0\rangle\}$ and $\varphi^{\prime}(k, 0)=\mathbf{T}$, is a system extending $\varphi$.

Definition 13.3. (A) Let DEF denote the set of all sets $X \subseteq \mathrm{HC}$, definable in HC (= all hereditarily countable sets) by $\in$-formulas with parameters in $\mathfrak{M} \cup\{\mathfrak{M}, \phi\}$. As DEF is countable, Lemma 13.2 allows to define an infinite system $\Phi: \omega \times \omega \rightarrow$ $\mathbf{M T}_{B}$ satisfying the requirements (2) and (3) of Definition 13.1 on the whole domain $k, m<\omega$, and also satisfying the following genericity condition: every set $\Delta \in \mathrm{DEF}$ is blocked by one of systems $\varphi \in \operatorname{Sys}\left(\mathfrak{S}^{\uparrow}\right), \varphi \subset \Phi$, in the sense that:

- either (I) $\varphi \in \Delta$,

— or (II) there is no system $\psi \in \operatorname{Sys}\left(\mathfrak{S}^{\uparrow}\right) \cap \Delta$ extending $\varphi$.

We let $\mathbf{T}_{m}^{k}=\Phi(k, m)$ for all $k, m<\omega$.

(B) The limit trees $\mathbf{L}^{k}=\bigwedge_{m} \mathbf{T}_{m}^{k}$, defined by $\left|\mathbf{L}^{k}\right|=B$ and $\mathbf{L}^{k}(\xi)=\bigcap_{m} \mathbf{T}_{m}^{k}(\xi)$ for all $\xi \in B$, belong to $\mathbf{M T}_{B}$ and satisfy $\mathbf{L}^{k} \leqslant_{m+1} \mathbf{T}_{m}^{k}$ for all $k, m$ by Lemma 7.5. 
Accordingly if $\xi \in B$ then $\mathbf{L}^{k}(\xi) \in \mathbf{L T}$ and $\mathbf{L}^{k}(\xi) \subseteq_{n} \mathbf{T}_{m}^{k}(\xi)$ for all $m$, where $n=\boldsymbol{\nu}_{m \xi}$ (Definition 7.3). This means $\mathbf{L}^{k}(\xi)(\rightarrow s) \subseteq \mathbf{T}_{m}^{k}(\xi)(\rightarrow s)$ for all $s \in 2^{n}$.

(C) If $\xi \in B$ then the set $Q_{\xi}=\left\{\sigma \cdot \mathbf{L}^{k}(\xi)(\rightarrow s): k<\omega \wedge \sigma, s \in 2^{<\omega}\right\}$ is a countable LT-forcing, see Example 6.2. We define a small multiforcing $\mathbf{Q}$ by $|\mathbf{Q}|=B$ and $\mathbf{Q}(\xi)=Q_{\xi}$ for all $\xi \in B$.

We'll check that the multiforcing $\mathbf{Q}$ satisfies all conditions of Definition 12.3. Note that $12.3(\mathrm{~A})$ directly holds by construction. The following lemma is obvious since option (II) of Definition 13.3(A) is impossible for dense sets $\Delta$. It will be a key ingredient in the verification of other conditions below.

Lemma 13.4. Let a set $\Delta \in \mathrm{DEF}, \Delta \subseteq \operatorname{Sys}\left(\mathfrak{S}^{\uparrow}\right)$, be dense in $\operatorname{Sys}\left(\mathfrak{S}^{\uparrow}\right)$, that is, every system in $\mathbf{S y s}\left(\boldsymbol{S}^{\uparrow}\right)$ is extendable to a system in $\Delta$. Then there exists a system $\varphi \in \Delta$ satisfying $\varphi \subset \Phi$.

Corollary 13.5. If $\mathbf{T} \in \mathfrak{S}^{\uparrow}$ then there is an index $k$ such that $\mathbf{L}^{k} \leqslant \mathbf{T}_{0}^{k}=\mathbf{T}$. If $\xi \in B$ and $T \in \mathbf{P}(\xi)$ then there is an index $k$ such that $\mathbf{L}^{k}(\xi) \subseteq \mathbf{T}_{0}^{k}(\xi)=T$.

Proof. Consider the set $\Delta$ of all system $\varphi \in \operatorname{Sys}\left(\mathfrak{S}^{\uparrow}\right)$ such that $\varphi(k, 0)=\mathbf{T}$ holds for at least one $k \in|\varphi|$. As $\mathbf{T} \in \mathfrak{S}^{\uparrow} \in \mathfrak{M}$, the set $\Delta$ belongs to DEF. We claim that $\Delta$ is dense in $\operatorname{Sys}\left(\mathfrak{S}^{\uparrow}\right)$. Indeed let $\varphi \in \operatorname{Sys}\left(\mathfrak{S}^{\uparrow}\right)$. Take any $k \notin|\varphi|$. By Lemma 13.2(ii) there is a system $\psi \in \operatorname{Sys}\left(\mathfrak{S}^{\uparrow}\right)$ extending $\varphi$ and satisfying $\langle k, 0\rangle \in \operatorname{dom} \psi$ and $\psi(k, 0)=\mathbf{T}$. Thus $\psi \in \Delta$, and the density is proved.

By Lemma 13.4, there is a system $\varphi \in \Delta, \varphi \subset \Phi$. Then $\mathbf{T}_{0}^{k}=\varphi(k, 0)=\mathbf{T}$ for some $k$. But $\mathbf{L}^{k}$ satisfies $\mathbf{L}^{k} \leqslant \mathbf{T}_{0}^{k}$ by $13.3(\mathrm{~B})$, as required.

To reduce the second claim to the first one, note that if $\xi \in B$ and $T \in \mathbf{P}(\xi)$ then by definition there is a multitree $\mathbf{T} \in \mathfrak{S}^{\uparrow}$ satisfying $\mathbf{T}(\xi)=T$.

\section{Verification of requirements}

We check conditions of Definition 12.3 for $\mathbf{Q}$ in the context of Section 13 .

Validation of 12.3(B). Fix $\xi \in B$. To check (1) of Definition 6.4 (the density of $\mathbf{Q}(\xi)$ in $\mathbf{Q}(\xi) \cup \mathbf{P}(\xi))$, let $T \in \mathbf{P}(\xi)$. Then $\mathbf{L}^{k}(\xi) \subseteq T$ for some $k$ by Corollary 13.5. But the tree $S=\mathbf{L}^{k}(\xi)$ belongs to $Q_{\xi}=\mathbf{Q}(\xi)$ by $13.3(\mathrm{C})$, as required.

Now assume that $\xi \in B$, a set $D \in \mathfrak{M}, D \subseteq \mathbf{P}(\xi)$ is pre-dense in $\mathbf{P}(\xi)$, and $U \in \mathbf{Q}(\xi)$. Prove $U \subseteq \subseteq^{\text {fin }} \cup D$. By definition, $U=\sigma \cdot \mathbf{L}^{k}(\xi)(\rightarrow s)$, where $k<\omega, \xi \in B$, and $s, \sigma \in 2^{<\omega}$. We can assume that $\sigma=\Lambda$, i.e., in fact just $U=$ $\mathbf{L}^{k}(\xi)(\rightarrow s)$. (The general case is reduced to $U=\mathbf{L}^{k}(\xi)(\rightarrow s)$ by the substitution of $\sigma \cdot D$ for $D$.) Furthermore, we can assume that $s=\Lambda$, i.e., $U=\mathbf{L}^{k}(\xi)$, because $\mathbf{L}^{k}(\xi)(\rightarrow s) \subseteq \mathbf{L}^{k}(\xi)$. Thus let $U=\mathbf{L}^{k}(\xi)$. The index $k$ will be fixed.

It follows, from the pre-density of $D$ and property 9.1(I) of the submultiforcing $\mathfrak{S}^{\uparrow}$, that the set $\mathbf{D} \in \mathfrak{M}$, of all multitrees $\mathbf{T} \in \mathfrak{S}^{\uparrow}$ satisfying $\mathbf{T}(\xi) \subseteq V$ for some $V \in D$, is itself open dense in $\mathfrak{S}^{\uparrow}$.

We claim that the set $\Delta \in \mathfrak{M}$ of all system $\varphi \in \operatorname{Sys}\left(\mathfrak{S}^{\uparrow}\right)$, such that $k \in|\varphi|$, and for every string $t \in 2^{n}$, where $n=\nu_{k}^{\varphi}$, the multitree $\varphi(k, n)(\Rightarrow t)$ belongs to 
$\mathbf{D}$, is dense in $\operatorname{Sys}\left(\mathfrak{S}^{\uparrow}\right)$. Indeed let $\varphi \in \operatorname{Sys}\left(\mathfrak{S}^{\uparrow}\right)$. By Lemma 13.2(ii), we assume that $k \in|\varphi|$, i.e., $n^{\prime}=\nu_{k}^{\varphi} \geq 0$. By definition the multitree $\mathbf{T}=\varphi\left(k, n^{\prime}\right)$ is an $n^{\prime}$-collage over $\mathfrak{S}^{\uparrow}$, and then, by Lemma 9.3(i), $n$-collage, too, where $n=n^{\prime}+1$. Then by Lemma 9.3(iii) there is a multitree $\mathbf{T}^{\prime} \in \mathbf{M} \mathbf{T}_{B}$, which is an $n$-collage over $\mathfrak{S}^{\uparrow}$ and satisfies $\mathbf{T}^{\prime} \leqslant n \quad \mathbf{T}$ and $\mathbf{T}^{\prime}(\Rightarrow t) \in \mathbf{D}$ for all $t \in 2^{n}$. Extend $\varphi$ to a system $\psi$ by $\operatorname{dom} \psi=\operatorname{dom} \varphi \cup\{\langle k, n\rangle\}$ and $\psi(k, n)=\mathbf{T}^{\prime}$; we have $\psi \in \Delta$.

Now by Lemma 13.4 there is a system $\varphi \in \Delta$ satisfying $\varphi \subset \Phi$. Then $\varphi(k, n)(\Rightarrow t)=\mathbf{T}_{n}^{k}(\Rightarrow t) \in \mathbf{D}$ for all $t \in 2^{n}$, where $n=\nu_{k}^{\varphi}$, thus $\mathbf{T}_{n}^{k} \subseteq^{\text {fd }} \bigvee \mathbf{D}$, hence $\mathbf{L}^{k} \subseteq{ }^{\text {fd }} \bigvee \mathbf{D}$. Therefore $U=\mathbf{L}^{k}(\xi) \subseteq{ }^{\text {fin }} \cup D$ by the definition of $\mathbf{D}$.

Validation of 12.3(C). Assume that $\mathbf{D} \in \mathfrak{M}, \mathbf{D} \subseteq \mathfrak{S}$ is open dense in $\mathfrak{S}$. Accordingly the set $\mathbf{D}^{\uparrow}=\left\{\mathbf{T}^{\uparrow}: \mathbf{T} \in \mathbf{D}\right\} \subseteq \mathfrak{S}^{\uparrow}$ is dense in $\mathfrak{S}^{\uparrow} .{ }^{6}$ By Corollary 13.5, it suffices to prove that $\mathbf{L}^{k} \subseteq \subseteq^{\mathrm{fd}} \bigvee \mathbf{D}^{\uparrow}$ for all $k<\omega$.

By the open-density of $\mathbf{D}^{\uparrow}$, the set $\Delta_{k} \in \mathfrak{M}$ of all systems $\varphi \in \operatorname{Sys}\left(\mathfrak{S}^{\uparrow}\right)$ such that $k \in|\varphi|$,and for every string $t \in 2^{n}$, where $n=\nu_{k}^{\varphi}$, the multitree $\varphi(k, n)(\Rightarrow t)$ belongs to $\mathbf{D}^{\uparrow}$, is dense in $\operatorname{Sys}\left(\mathfrak{S}^{\uparrow}\right)$. (See the verification of 12.3(B) above.) By Lemma 13.4 there exists a system $\varphi \in \Delta_{k}$ satisfying $\varphi \subset \Phi$. Then $\varphi(k, n)(\Rightarrow t)=\mathbf{T}_{n}^{k}(\Rightarrow t) \in \mathbf{D}$ for all $t \in 2^{n}$, where $n=\nu_{k}^{\varphi}$, that is, $\mathbf{T}_{n}^{k} \subseteq{ }^{\mathrm{fd}} \bigvee \mathbf{D}^{\uparrow}$ holds, hence $\mathbf{L}^{k} \subseteq{ }^{\mathrm{fd}} \bigvee \mathbf{D}^{\uparrow}$, as required.

Validation of 12.3(D). Let $\mathbf{T} \in \mathfrak{S}, \xi \in C=|\mathbf{T}|, \mathbf{c} \in \mathrm{CCF}_{C} \cap \mathfrak{M}$, and $f=f^{\mathbf{c}}$ (a continuous map $\left(2^{\omega}\right)^{C} \rightarrow 2^{\omega}$ ). The multitree $\mathbf{T}^{\uparrow}=\mathbf{T} \uparrow B$ belongs to $\mathfrak{S}^{\uparrow}$, and the map $f^{\uparrow}(x)=f\left(x\lceil C):\left(2^{\omega}\right)^{B} \rightarrow 2^{\omega}\right.$ is continuous. In terms of Section 11, we can assume that $\left(^{*}\right)$ there is no multitree $\mathbf{T}^{\prime} \in \mathfrak{S}^{\uparrow}, \mathbf{T}^{\prime} \leqslant \mathbf{T}^{\uparrow}$, such that $f^{\uparrow}$ is simple for $\xi$ on $\mathbf{T}^{\prime}$. Indeed otherwise using Corollary 13.5 we get a multitree $\mathbf{S}$ of the form $\mathbf{L}^{k}$, satisfying $\mathbf{L}^{k} \leqslant \mathbf{T}^{\prime}$, and hence (i) of $12.3(\mathrm{D})$.

Now assuming (*) we accordingly prove that any multitree $\mathbf{S}=\mathbf{L}^{k}$ with $\mathbf{L}^{k} \leqslant$ $\mathbf{T}_{0}^{k}=\mathbf{T}^{\uparrow}$ satisfies (ii) of $12.3(\mathrm{D})$. Let $U \in \mathbf{Q}(\xi)=Q_{\xi}$, and we have to prove that $f^{\uparrow}(x) \notin[U]$ for all $x \in\left[\mathbf{L}^{k}\right]$. By definition, $U=\tau \cdot \mathbf{L}^{\ell}(\xi)(\Rightarrow s)$, where $\tau, s \in 2^{<\omega}$ and $\ell<\omega$. Now, as $\mathbf{L}^{\ell}(\xi)(\Rightarrow s) \subseteq \mathbf{L}^{\ell}(\xi)$, we can assume that $s=\Lambda$, that is, $U=\tau \cdot \mathbf{L}^{\ell}(\xi)$. Moreover we can assume that $\tau=\Lambda$, i.e., $U=\mathbf{L}^{\ell}(\xi)$; otherwise consider the map $f^{\prime}(x)=\tau \cdot f^{\uparrow}(x)$ instead of $f^{\uparrow}$.

Thus we fix an index $\ell<\omega$ and prove that $\left[\mathbf{L}^{\ell}(\xi)\right] \cap f^{\uparrow " ~}\left[\mathbf{L}^{k}\right]=\varnothing$.

Case 1: $\ell \neq k$. Consider the set $\Delta$ of all systems $\varphi \in \operatorname{Sys}\left(\mathfrak{S}^{\uparrow}\right)$ such that $k, \ell \in$ $|\varphi|$, that is, $m=\nu_{k}^{\varphi} \geq 0$ and $n=\nu_{\ell}^{\varphi} \geq 0$, and $[\varphi(\ell, n)(\xi)] \cap f^{\uparrow " ~}[\varphi(k, m)]=\varnothing$.

Lemma 14.1. The set $\Delta$ is dense in $\operatorname{Sys}\left(\mathfrak{S}^{\uparrow}\right)$.

Proof (Lemma). Let $\varphi \in \operatorname{Sys}\left(\mathfrak{S}^{\uparrow}\right)$. By Lemma 13.2(ii), we can assume that $k, \ell \in|\varphi|$, that is, $n^{\prime}=\nu_{\ell}^{\varphi} \geq 0$ and $m^{\prime}=\nu_{k}^{\varphi} \geq 0$. By definition, the multitree $\mathbf{R}^{\prime}=\varphi\left(k, m^{\prime}\right)$ is an $m^{\prime}$-collage over $\mathfrak{S}^{\uparrow}$, and then $m$-collage, too, by Lemma 9.3(i), where $m=m^{\prime}+1$.

${ }^{6}$ To prove the openness let $\mathbf{T} \in \mathbf{D}$. Then $\mathbf{T}^{\uparrow} \in \mathbf{D}^{\uparrow}, \mathbf{S} \in \mathfrak{S}$, and $\mathbf{S}^{\uparrow} \leqslant \mathbf{T}^{\uparrow}$. We cannot assert directly that $\mathbf{S} \leqslant \mathbf{T}$. However the multitree $\mathbf{S}^{\prime}=\mathbf{S} \uparrow(|\mathbf{T}| \cup|\mathbf{S}|)$ also belongs to $\mathfrak{S}$ by Definition 9.1(III). Note that $\mathbf{S}^{\uparrow} \leqslant \mathbf{T}^{\uparrow}$ easily implies $\mathbf{S}^{\prime} \leqslant \mathbf{T}$. Therefore $\mathbf{S}^{\prime} \in \mathbf{D}$, since $\mathbf{D}$ is open. We conclude that $\mathbf{S}^{\uparrow}=\mathbf{S}^{\prime \uparrow} \in \mathbf{D}^{\uparrow}$. 
Further, we can assume that $\phi\left(n^{\prime}\right)=\xi$, for if not then take the least number $n^{\prime \prime}>n^{\prime}$ satisfying $\phi\left(n^{\prime \prime}\right)=\xi$, and trivially extend the system $\varphi$ by $\varphi(\ell, j)=$ $\varphi\left(\ell, n^{\prime}\right)$ for all $\ell$ with $n^{\prime}<\ell \leq n^{\prime \prime}$. As above, the multitree $\mathbf{Z}^{\prime}=\varphi\left(\ell, n^{\prime}\right)$ is an $n^{\prime}$-collage over $\mathfrak{S}^{\uparrow}$, and hence $n$-collage, where $n=n^{\prime}+1$. It follows that $\mathbf{Z}^{\prime}(\Rightarrow \sigma) \in \mathfrak{S}^{\uparrow}$ for all $\sigma \in 2^{n}$. In particular $\mathbf{Z}^{\prime}(\Rightarrow \sigma)(\xi) \in \mathbf{P}(\xi)$ for $\sigma \in 2^{n}$. Yet $\mathbf{Z}^{\prime}(\Rightarrow \sigma)(\xi)=\mathbf{Z}^{\prime}(\xi)\left(\rightarrow \sigma\lceil\xi)\right.$ by Definition 7.3 , where $\sigma\left\lceil\xi \in 2^{\nu}\right.$ and $\nu=\boldsymbol{\nu}_{m \xi}$. Therefore the tree $Z^{\prime}=\mathbf{Z}^{\prime}(\xi)$ is an $\nu$-collage over $\mathbf{P}(\xi)$.

By Theorem 11.2(i), there exist a multitree $\mathbf{R} \in \mathbf{M T}_{B}$ and a tree $Z \in \mathbf{L T}$ such that $\mathbf{R} \leqslant_{m} \mathbf{R}^{\prime}, Z \subseteq_{\nu} Z^{\prime}, \mathbf{R}$ is an $m$-collage over $\mathfrak{S}^{\uparrow}, Z$ is an $\nu$-collage over $\mathfrak{S}(\xi)$, and $[Z] \cap f^{\uparrow " ~}[\mathbf{R}]=\varnothing$. Define a multitree $\mathbf{Z} \in \mathbf{M} \mathbf{T}_{B}$ so that $\mathbf{Z}(\xi)=Z$ and $\mathbf{Z}(\eta)=\mathbf{Z}^{\prime}(\eta)$ for all $\eta \in B, \eta \neq \xi$.

Sublemma 14.2. $\mathbf{Z}$ is an $n$-collage over $\mathfrak{S}^{\uparrow}$ and $\mathbf{Z} \leqslant n \mathbf{Z}^{\prime}$.

Proof. Let $\sigma=\tau^{\frown} i \in 2^{n}$, where $\tau \in 2^{n^{\prime}}$ and $i=0,1$. The strings $\sigma\left\lfloor\eta \in 2^{\boldsymbol{\nu}_{n \eta}}\right.$ and $\tau\left\lceil\eta \in 2^{\nu_{n^{\prime} \eta}}\right.$ (Definition 7.3) are connected so that: $\sigma\lfloor\eta=\tau\lceil\eta$ for $\eta \neq \xi$, but $\sigma\left[\xi=\left(\tau[\xi)^{\frown} i\right.\right.$, since $\phi\left(n^{\prime}\right)=\xi$ and $n=n^{\prime}+1$. It follows that

$$
\mathbf{Z}(\Rightarrow \sigma)(\eta)=\mathbf{Z}(\eta)\left(\rightarrow \sigma\lceil\eta)=\mathbf{Z}^{\prime}(\eta)\left(\rightarrow \sigma\lceil\eta)=\mathbf{Z}^{\prime}(\Rightarrow \sigma)(\eta)\right.\right.
$$

for $\eta \neq \xi$, that is, $\mathbf{Z}(\Rightarrow \sigma) \uparrow(B \backslash\{\xi\})=\mathbf{Z}^{\prime}(\Rightarrow \sigma) \uparrow(B \backslash\{\xi\})$. Further, $\mathbf{Z}(\Rightarrow \sigma)(\xi)=$ $\mathbf{Z}(\xi)(\rightarrow \sigma\lceil\xi)=Z(\rightarrow \sigma\lceil\xi)=Z(\rightarrow \tau\lceil\xi)(\rightarrow i) \in \mathbf{P}(\xi)$, since $Z$ is a $\nu$-collage over $\mathfrak{S}(\xi)$. This implies $\mathbf{Z}(\Rightarrow \sigma) \in \mathfrak{S}^{\uparrow}$ by the property $9.1(\mathrm{I})$ of submultiforcings. As $\sigma \in 2^{n}$ is arbitrary, $\mathbf{Z}$ is a $n$-collage over $\mathfrak{S}^{\uparrow}$.

To establish $\mathbf{Z} \leqslant n \mathbf{Z}^{\prime}$, we need (in the same notation) to prove $\mathbf{Z}(\Rightarrow \sigma) \leqslant$ $\mathbf{Z}^{\prime}(\Rightarrow \sigma)$ for all $\sigma \in 2^{n}$, that is, $\mathbf{Z}(\Rightarrow \sigma)(\eta) \subseteq \mathbf{Z}^{\prime}(\Rightarrow \sigma)(\eta)$ for all $\eta \in B$. If $\eta \neq \xi$ then simply $\mathbf{Z}(\Rightarrow \sigma)(\eta) \subseteq \mathbf{Z}^{\prime}(\Rightarrow \sigma)(\eta)$, as above. Further, we have $\mathbf{Z}(\Rightarrow \sigma)(\xi)=$ $Z(\rightarrow s)$ and $\mathbf{Z}^{\prime}(\Rightarrow \sigma)(\xi)=Z^{\prime}(\rightarrow s)$, where $s=\sigma\left[\xi \in 2^{\nu}, \nu=\boldsymbol{\nu}_{m \xi}\right.$. But $Z \subseteq_{\nu} Z^{\prime}$ by construction, hence $Z(\rightarrow s) \subseteq Z^{\prime}(\rightarrow s)$, or equivalently, $\mathbf{Z}(\Rightarrow \sigma)(\xi) \subseteq \mathbf{Z}^{\prime}(\Rightarrow \sigma)(\xi)$. Thus $\mathbf{Z}(\Rightarrow \sigma)(\eta) \subseteq \mathbf{Z}^{\prime}(\Rightarrow \sigma)(\eta)$ for all $\eta \in B$, that is, $\mathbf{Z}(\Rightarrow \sigma) \leqslant \mathbf{Z}^{\prime}(\Rightarrow \sigma)$, as required.

(Sublemma)

Coming back to the lemma, we extend $\varphi$ to a system $\psi$ with $\operatorname{dom} \psi=\operatorname{dom} \varphi$, $\nu_{k}^{\varphi}=m, \nu_{\ell}^{\varphi}=n, \psi(k, m)=\mathbf{R}$, and $\psi(\ell, n)=\mathbf{Z}$ (just two new values). Thus $\psi$ is a system in $\operatorname{Sys}\left(\mathfrak{S}^{\uparrow}\right)$. Indeed $\psi(k, m)=\mathbf{R}$, one of the two new terms relatively to $\varphi$, is an $m$-collage over $\mathfrak{S}^{\uparrow}$, and $\mathbf{R} \leqslant_{m} \mathbf{R}^{\prime}=\varphi\left(k, m^{\prime}\right)$, where $m=m^{\prime}+1$, as required by 13.1(3). Similarly for $\psi(\ell, n)=\mathbf{Z}$ the other new term. Thus $\psi \in \operatorname{Sys}\left(\mathfrak{S}^{\uparrow}\right)$ and clearly $\varphi \preccurlyeq \psi$. Finally $[Z] \cap f^{\uparrow " ~}[\mathbf{R}]=\varnothing$ by construction, hence $\psi \in \Delta$. This ends the proof of the density of $\Delta$.

(Lemma)

Now Corollary 13.4 yields a system $\varphi \in \operatorname{Sys}\left(\mathfrak{S}^{\uparrow}\right), \varphi \subset \Phi$. Then $k, \ell \in|\varphi|$, hence $m=\nu_{k}^{\varphi} \geq 0$ and $n=\nu_{\ell}^{\varphi} \geq 0$, and multitrees $\mathbf{T}_{m}^{k}=\varphi(k, m), \mathbf{T}_{n}^{\ell}=\varphi(\ell, n)$ satisfy $\left[\mathbf{T}_{n}^{\ell}(\xi)\right] \cap f^{\uparrow " ~}\left[\mathbf{T}_{m}^{k}\right]=\varnothing$ by the definition of $\Delta$, therefore $\left[\mathbf{L}^{k}(\xi)\right] \cap f^{\uparrow " ~}\left[\mathbf{L}^{k}\right]=$ $\varnothing$, because $\mathbf{L}^{k} \subseteq \mathbf{T}_{m}^{k}$, as required.

Case 2: $\ell=k$. Consider the set $\Delta$ of all systems $\varphi \in \operatorname{Sys}\left(\mathfrak{S}^{\uparrow}\right)$ such that $k \in|\varphi|$ (and then $m=\nu_{k}^{\varphi} \geq 0$ ) and $[\varphi(k, m)(\xi)] \cap f^{\uparrow "}[\varphi(k, m)]=\varnothing$. We don't 
claim that $\Delta$ is dense. However by Definition 13.3 there is a systems $\varphi \in \operatorname{Sys}\left(\mathfrak{S}^{\uparrow}\right)$, $\varphi \subset \Phi$, blocking $\Delta$ in the sense of $13.3(\mathrm{~A}),(\mathrm{I}) \vee(\mathrm{II})$.

We now assert that 13.3(A)(II) is impossible for $\varphi$. Indeed let $m^{\prime}=\nu_{k}^{\varphi}$ and $\mathbf{R}^{\prime}=\varphi\left(k, m^{\prime}\right)=\Phi\left(k, m^{\prime}\right)=\mathbf{T}_{m^{\prime}}^{k}$. Then $\mathbf{R} \subseteq \mathfrak{S}^{\uparrow}=\mathbf{T}_{0}^{k}$, and hence by $\left(^{*}\right)$ (in the beginning of validation of $12.3(\mathrm{D}))$, if $\mathbf{T}^{\prime} \in \mathfrak{S}^{\uparrow}, \mathbf{T}^{\prime} \leqslant \mathbf{R}^{\prime}$, then the map $f^{\uparrow}$ is not siple for $\xi$ on $\mathbf{T}^{\prime}$. Therefore by Theorem 11.2(ii) there is a multitree $\mathbf{R} \in \mathbf{M T}(\mathbf{P})$, which is a $m$-collage over $\mathfrak{S}^{\uparrow}$, where $m=m^{\prime}+1$, and satisfies $\mathbf{R} \leqslant_{m} \mathbf{R}^{\prime}$ and $[\mathbf{R}(\xi)] \cap f^{\uparrow} "[\mathbf{R}]=\varnothing$. As in Case 1 , we can extend $\varphi$ to a system $\psi \in \operatorname{Sys}\left(\mathfrak{S}^{\uparrow}\right)$ with the only one new term $\psi(k, m)=\mathbf{R}$, and then $\psi \in \Delta$ by the choice of $\mathbf{R}$. This proves that 13.3(A)(II) cannot happen for $\varphi$.

Thus 13.3(A)(I) takes place, that is, $\varphi \in \Delta$. It follows that $[\varphi(k, m)(\xi)] \cap$ $f^{\uparrow "}[\varphi(k, m)]=\varnothing$, hence $\left[\mathbf{T}_{m}^{k}(\xi)\right] \cap f^{\uparrow "}\left[\mathbf{T}_{m}^{k}\right]=\varnothing$. This implies $\left[\mathbf{L}^{k}(\xi)\right] \cap f^{\uparrow "}\left[\mathbf{L}^{k}\right]=$ $\varnothing$, since $\mathbf{L}^{k} \leqslant \mathbf{T}_{m}^{k}$, as required.

\section{The forcing}

We argue in the constructible universe $\mathbf{L}$ in this section.

We begin with some definitions related to sequences of multiforcings.

First of all, we somewhat generalize the definition of $\sqsubset_{\mathfrak{M}}$ in 12.3. Given small multiforcings $\mathbf{P}, \mathbf{Q}$ and a model $\mathfrak{M}$, we define $\mathbf{P} \sqsubset_{\mathfrak{M}}^{+} \mathbf{Q}$, when $|\mathbf{P}| \subseteq|\mathbf{Q}|$ and $\mathbf{P} \sqsubset_{\mathfrak{M}}\left(\mathbf{Q}\lceil|\mathbf{P}|)\right.$ in the sense of 12.3. If $\overrightarrow{\mathbf{P}}=\left\langle\mathbf{P}_{\alpha}\right\rangle_{\alpha<\lambda}\left(\lambda<\omega_{1}\right)$ is a sequence of small multiforcings $\mathbf{P}_{\alpha}$ then:

(a) $\mathfrak{M}(\overrightarrow{\mathbf{P}})$ will denote the least transitive model of $\mathbf{Z F C}$ (see Definition 12.3) of the form $\mathbf{L}_{\gamma}$, containing $\overrightarrow{\mathbf{P}}$ (and then all multiforcings $\mathbf{P}_{\nu}$ ), in which $\lambda$ and all sets $\left|\mathbf{P}_{\nu}\right|$ and forcings $\mathbf{P}_{\nu}(\xi)\left(\xi \in\left|\mathbf{P}_{\nu}\right|\right)$ are at most countable,

(b) a multiforcing $\mathbf{P}=\bigcup^{\text {cw }} \overrightarrow{\mathbf{P}}=\bigcup_{\nu<\lambda}^{\text {cw }} \mathbf{P}_{\nu}$ (componentwise union) is defined by $|\mathbf{P}|=\bigcup_{\nu<\lambda}\left|\mathbf{P}_{\nu}\right|$ and $\mathbf{P}(\xi)=\bigcup_{\xi<\nu<\lambda, \xi \in\left|\mathbf{P}_{\nu}\right|} \mathbf{P}_{\nu}(\xi)$ for all $\xi \in|\mathbf{P}|$.

Definition 15.1 (in $\mathbf{L}$ ). Let $\lambda \leq \omega_{1} \cdot \overrightarrow{\mathbf{M F}}_{\lambda}$ is the set of all $\lambda$-sequences $\overrightarrow{\mathbf{P}}=$ $\left\langle\mathbf{P}_{\nu}\right\rangle_{\nu<\lambda}$ of small multiforcings $\mathbf{P}_{\nu}$, such that for each $\nu<\lambda$ :

1) $\left|\mathbf{P}_{\nu}\right|=\nu+1$,

2) $\mathbf{P}_{\nu}(\nu)$ contains the tree $2^{<\omega}$ (regularity), and

3) $\bigcup_{\mu<\nu}^{\mathrm{cw}} \mathbf{P}_{\mu} \sqsubset_{\mathfrak{M}(\overrightarrow{\mathbf{P}}\lceil\nu)}^{+} \mathbf{P}_{\nu}$.

We put $\overrightarrow{\mathbf{M F}}=\bigcup_{\lambda<\omega_{1}} \overrightarrow{\mathbf{M F}}_{\lambda}$.

The set $\overrightarrow{\mathbf{M F}} \cup \overrightarrow{\mathbf{M F}}_{\omega_{1}}$ is ordered by the extension relations $\subset, \subseteq$.

Lemma 15.2 (in $\mathbf{L}$ ). Assume that $\kappa<\lambda<\omega_{1}$, and $\overrightarrow{\mathbf{P}}=\left\langle\mathbf{P}_{\nu}\right\rangle_{\nu<\kappa}$ is a sequence in $\overrightarrow{\mathbf{M F}}_{\kappa}$. Then:

(i) $\mathbf{P}=\bigcup^{\mathrm{cw}} \overrightarrow{\mathbf{P}}$ is a small regular multiforcing and $|\mathbf{P}|=\kappa$;

(ii) there is a sequence $\overrightarrow{\mathbf{Q}} \in \overrightarrow{\mathbf{M F}}$ such that $\operatorname{dom} \overrightarrow{\mathbf{Q}}=\lambda$ and $\overrightarrow{\mathbf{P}} \subset \overrightarrow{\mathbf{Q}}$. 
Proof. (i) By definition, $\mathbf{P}(\xi)=\bigcup_{\xi \leq \nu<\kappa} \mathbf{P}_{\nu}(\xi)$. The first term $\mathbf{P}_{\xi}(\xi)$ in the union contains $2^{<\omega}$, so that the regularity follows.

(ii) We define multiforcings $\mathbf{P}_{\alpha}, \kappa \leq \alpha<\lambda$, by induction on $\alpha$. Assume that all terms $\mathbf{P}_{\nu}, \kappa \leq \nu<\alpha$, are defined, and the sequence obtained $\overrightarrow{\mathbf{Q}}=\left\langle\mathbf{P}_{\mu}\right\rangle_{\mu<\alpha}$ belongs to $\overrightarrow{\mathbf{M F}}_{\alpha}$. Then $\mathbf{P}^{\prime}=\bigcup^{\text {cw }} \overrightarrow{\mathbf{Q}}=\bigcup_{\mu<\alpha}^{\mathrm{cw}} \mathbf{P}_{\mu}$ is a small regular multiforcing with $\left|\mathbf{P}^{\prime}\right|=\alpha$ by (i), and $\mathbf{P}^{\prime} \in \mathfrak{M}=\mathfrak{M}(\overrightarrow{\mathbf{Q}})$. Theorem 12.4 gives a small multiforcing $\mathbf{Q}$ satisfying $|\mathbf{Q}|=\alpha$ and $\mathbf{P}^{\prime} \sqsubset_{\mathfrak{M}} \mathbf{Q}$. Define a small multiforcing $\mathbf{P}_{\alpha}$ so that $\left|\mathbf{P}_{\alpha}\right|=\alpha+1, \mathbf{P}_{\alpha}(\xi)=\mathbf{Q}(\xi)$ for all $\xi<\alpha$, and, to fix the regularity, $\mathbf{P}_{\alpha}(\alpha)=P_{\text {coh }}$ (see Example 6.2), hence $2^{<\omega} \in \mathbf{P}_{\alpha}(\alpha)$.

Definition 15.3 (key definition). A sequence $\overrightarrow{\mathbf{P}} \in \overrightarrow{\mathbf{M F}}$ blocks a set $W \subseteq \overrightarrow{\mathbf{M F}}$, if either $\overrightarrow{\mathbf{P}} \in W$ (positive block) or there is no sequence $\overrightarrow{\mathbf{Q}} \in W$ with $\overrightarrow{\overrightarrow{\mathbf{P}}} \subseteq \overrightarrow{\mathbf{Q}}$ (negative block).

Approaching the blocking sequence theorem, we recall that $\mathrm{HC}$ is the set of all hereditarily countable sets, so that $\mathrm{HC}=\mathbf{L}_{\omega_{1}}$ in $\mathbf{L}$. See [3, Part 2, Chapter 5.4] on definability classes $\Sigma_{n}^{X}, \Pi_{n}^{X}, \Delta_{n}^{X}$ for any set $X$, in particular, on $\Sigma_{n}^{\mathrm{HC}}, \Pi_{n}^{\mathrm{HC}}, \Delta_{n}^{\mathrm{HC}}$ for $X=\mathrm{HC}$ in $[17$, sections 8,9$]$ or elsewhere.

Theorem 15.4 (in $\mathbf{L}$ ). If $\mathrm{n} \geq 3$ then there is a sequence $\overrightarrow{\mathbb{P}}=\left\langle\mathbb{P}_{\alpha}\right\rangle_{\alpha<\omega_{1}} \in \overrightarrow{\mathbf{M F}}_{\omega_{1}}$, satisfying the following two conditions:

(i) $\overrightarrow{\mathbb{P}}$ itself, as a set of pairs $\left\langle\alpha, \mathbb{P}_{\alpha}\right\rangle$, belongs to $\Delta_{\curvearrowleft-1}^{\mathrm{HC}}$;

(ii) (genericity of $\overrightarrow{\mathbb{P}}$ with respect to $\Sigma_{\mathfrak{m}-2}^{\mathrm{HC}}(\mathrm{HC})$ sets) if $W \subseteq \overrightarrow{\mathbf{M F}}$ is a $\Sigma_{\mathfrak{m}-2}^{\mathrm{HC}}(\mathrm{HC})$ set (i.e., parameters in $\mathrm{HC}$ admitted in the defining formula), then there is $\gamma<\omega_{1}$ such that the restricted sequence $\overrightarrow{\mathbb{P}} \mid \gamma=\left\langle\mathbb{P}_{\alpha}\right\rangle_{\alpha<\gamma} \in \overrightarrow{\mathbf{M F}}$ blocks $W$.

Proof. Let $\leqslant_{\mathbf{L}}$ denote a canonical well-ordering of $\mathbf{L}$; its restriction to $\mathrm{HC}=\mathbf{L}_{\omega_{1}}$ is a $\Delta_{1}^{\mathrm{HC}}$ relation. There exists a universal $\Sigma_{\mathfrak{m}-2}^{\mathrm{HC}}$ set $\mathfrak{U} \subseteq \omega_{1} \times \mathrm{HC}$. Thus $\mathfrak{U}$ belongs to $\Sigma_{\mathrm{m}-2}^{\mathrm{HC}}$ (parameter-free $\Sigma_{\mathrm{m}-2}$ definability in $\mathrm{HC}$ ), and for any $\Sigma_{\mathrm{m}-2}^{\mathrm{HC}}(\mathrm{HC})$ set $X \subseteq \mathrm{HC}$ (definable in $\mathrm{HC}$ by a $\Sigma_{\mathrm{n}-2}$ formula with parameters in $\mathrm{HC}$ ) there is an ordinal $\alpha<\omega_{1}$ satisfying $X=\mathfrak{U}_{\alpha}$, where $\mathfrak{U}_{\alpha}=\{x:\langle\alpha, x\rangle \in \mathfrak{U}\}$. The choice of $\omega_{1}$ as the domain of parameters is validated by the hypothesis $\mathbf{V}=\mathbf{L}$, which is accepted in this section and implies the existence of a $\Delta_{1}^{\mathrm{HC}}$ surjection $\omega_{1} \stackrel{\text { onto }}{\longrightarrow} \mathrm{HC}$.

Coming back to Definition 15.3 , note that if $\overrightarrow{\mathbf{P}} \in \overrightarrow{\mathbf{M F}}$ and $W \subseteq \overrightarrow{\mathbf{M F}}$ is any set then there is a sequence $\overrightarrow{\mathbf{Q}} \in \overrightarrow{\mathbf{M F}}$, satisfying $\overrightarrow{\mathbf{P}} \subset \overrightarrow{\mathbf{Q}}$ and blocking $W$. We define $\overrightarrow{\mathbf{Q}}_{\alpha} \in \overrightarrow{\mathbf{M F}}$ by induction on $\alpha<\omega_{1}$ so that $\overrightarrow{\mathbf{Q}}_{0}=\varnothing, \overrightarrow{\mathbf{Q}}_{\lambda}=\bigcup_{\alpha<\lambda} \overrightarrow{\mathbf{Q}}_{\alpha}$ for limit $\lambda$, and each $\overrightarrow{\mathbf{Q}}_{\alpha+1}$ is the $\leqslant_{\mathbf{L}}$-least sequence $\overrightarrow{\mathbf{Q}} \in \overrightarrow{\mathbf{M F}}$ satisfying $\overrightarrow{\mathbf{P}} \subset \overrightarrow{\mathbf{Q}}$ and blocking $\mathfrak{U}_{\alpha}$. Then $\overrightarrow{\mathbb{P}}=\bigcup_{\alpha<\omega_{1}} \overrightarrow{\mathbf{Q}}_{\alpha} \in \overrightarrow{\mathbf{M F}}_{\omega_{1}}$.

Now (ii) holds by construction, while (i) admits a routine verification based on the fact that $\overrightarrow{\mathbf{M F}} \in \Delta_{1}^{\mathrm{HC}}$.

Definition 15.5 (in L). Fix a number $n \geq 3$, for which Theorem 2.1 is proved. Fix a sequence $\overrightarrow{\mathbb{P}}=\left\langle\mathbb{P}_{\alpha}\right\rangle_{\alpha<\omega_{1}} \in \overrightarrow{\mathbf{M F}}_{\omega_{1}}$ which Theorem 15.4 provides for this $\mathrm{n}$. 
We put $\mathbb{P}=\bigcup_{\alpha<\omega_{1}}^{\mathrm{cw}} \mathbb{P}_{\alpha}$. Thus $\mathbb{P}$ is a multiforcing, $|\mathbb{P}|=\omega_{1}$, and $\mathbb{P}(\xi)=$ $\bigcup_{\xi \leq \alpha<\omega_{1}} \mathbb{P}_{\alpha}(\xi)$ for all $\xi<\omega_{1}$. By construction, each set $\mathbb{P}_{\alpha}$ is a small multiforcing satisfying $\left|\mathbb{P}_{\alpha}\right|=\alpha+1$, while each component $\mathbb{P}_{\alpha}(\xi)\left(\xi \leq \alpha<\omega_{1}\right)$ is a countable LT-forcing. It follows that if $\alpha<\omega_{1}$ then the multiforcing $\mathbb{P}_{<\alpha}=\bigcup_{\nu<\alpha}^{c \mathrm{r}} \mathbb{P}_{\nu}$ satisfies $\left|\mathbb{P}_{<\alpha}\right|=\alpha$. In addition, since $\overrightarrow{\mathbb{P}} \in \overrightarrow{\mathbf{M F}}_{\omega_{1}}$, we have

(*) $\mathbb{P}_{<\alpha} \sqsubset_{\mathfrak{M}_{\alpha}}^{+} \mathbb{P}_{\alpha}$, that is, $\mathbb{P}_{<\alpha} \sqsubset_{\mathfrak{M}_{\alpha}} \mathbb{P}_{\alpha}\lceil\alpha$ - for all $\alpha$,

where $\mathfrak{M}_{\alpha}=\mathfrak{M}(\overrightarrow{\mathbb{P}} \mid \alpha)$. The submultiforcing $\mathfrak{S}_{\alpha}=\mathfrak{S}\left(\mathbb{P}_{<\alpha}\right)$ in $\mathbf{M T}\left(\mathbb{P}_{<\alpha}\right)$ (see Definition 12.3) will also be considered.

The set $\square=\mathbf{M T}(\mathbb{P})$ will be used in the proof of Theorem 2.1 as a forcing notion. It is naturally identified with the countable-support product $\prod_{\xi<\omega_{1}} \mathbb{P}(\xi)$ (in $\mathbf{L}$ ). The sets $\mathbb{P}$ and $\square$ belong to $\mathbf{L}$ by construction.

The next theorem shows that $\mathbb{\square}$-generic extensions of $\mathbf{L}$ are models for Theorem 2.2. Therefore Theorem 15.6 implies Theorem 2.2 (and Theorem 2.1 as well).

Theorem 15.6. Under the conditions of Definition 15.5, let $\mathbb{G} \subseteq \square$ be a generic filter over $\mathbf{L}$. Then the following holds in $\mathbf{L}[\mathbb{G}]$ :

(i) condition (i) of Theorem 2.2;

(ii) condition (ii) of Theorem 2.2.

To prove Theorem 15.6, we explore properties of the forcing notion $\square$ and related generic extensions in Sections 16-18, then establish (i) of Theorem 15.6 in Section 19, and finally (ii) in Section 22 with the help of a special approximating forcing relation forc.

\section{Key forcing properties}

Here we study $\square$ as the forcing notion. We argue under the conditions and notation of Definition 15.5.

Definition $\mathbf{1 6 . 1}$ (in $\mathbf{L}$ ). If $C \subseteq \omega_{1}$ then we define the subproduct $\square \backslash C=$ $\mathbf{M T}(\mathbb{P} \mid C)=\{\mathbf{T} \in \mathbb{\square}:|\mathbf{T}| \subseteq C\}=\prod_{\xi \in C} \mathbb{P}(\xi)$ with countable support. Then $\square$ can be identified with $\left(\square\lceil C) \times\left(\square \uparrow\left(\omega_{1}^{\mathbf{L}} \backslash C\right)\right)\right.$.

If $C \subseteq \omega_{1}$ is at most countable (in $\mathbf{L}$ ), then by the regularity of $\mathbb{P}$ the set $\square \uparrow C$ can be identified with $\rrbracket_{C}=\{\mathbf{T} \in \mathbb{\square}:|\mathbf{T}|=C\}$.

If $C=\{\xi\}, \xi<\omega_{1}^{\mathbf{L}}$, then $\square \uparrow\{\xi\}$ is naturally identified with $\mathbb{P}(\xi)$, and then $\square$ is identified with $\mathbb{P}(\xi) \times \llbracket \uparrow C_{\neq \xi}$, where $C_{\neq \xi}=\omega_{1}^{\mathbf{L}} \backslash\{\xi\}$.

Lemma 16.2. If $\xi \leq \alpha<\gamma<\omega_{1}$ then $\mathbb{P}_{\alpha}(\xi) \sqsubset \mathbb{P}_{\gamma}(\xi)$ in the sense of 6.4. Therefore each $\mathbb{P}_{\alpha}(\xi)$ is pre-dense in $\mathbb{P}(\xi)=\bigcup_{\alpha \geq \xi} \mathbb{P}_{\alpha}(\xi)$ by Lemma 6.5(iii).

Proof. Arguing by induction, suppose that $\mathbb{P}_{\mu}(\xi) \sqsubset \mathbb{P}_{\nu}(\xi)$ is established for all $\xi \leq \mu<\nu<\gamma$. Lemma 6.5(iii) implies that the set $\mathbb{P}_{\alpha}(\xi)$ is pre-dense in 
$\bigcup_{\xi \leq \nu<\gamma} \mathbb{P}_{\nu}(\xi)$. The multiforcing $\mathbf{Q}=\mathbb{P}_{\gamma}\left\lceil\gamma\right.$ satisfies $\mathbb{P}_{<\gamma} \sqsubset_{\mathfrak{M}_{\gamma}} \mathbf{Q}$ by $15.5(*)$. By Definition 12.3, this includes the condition $\mathbb{P}_{<\gamma}(\xi) \sqsubset_{\mathfrak{M}_{\gamma}} \mathbf{Q}(\xi)$. Then immediately $\mathbf{Q}(\xi)$ is dense in $\mathbb{P}_{<\gamma}(\xi) \cup \mathbf{Q}(\xi)$. However $\mathbf{Q}(\xi)=\mathbb{P}_{\gamma}(\xi)$ while $\mathbb{P}_{<\gamma}(\xi)=\bigcup_{\xi<\nu<\gamma} \mathbb{P}_{\nu}(\xi)$. Therefore, first, $\mathbb{P}_{\gamma}(\xi)$ is dense in $\mathbb{P}_{\alpha}(\xi) \cup \mathbb{P}_{\gamma}(\xi)$, thus we have (1) of Definition 6.4. And second, as the set $\mathbb{P}_{\alpha}(\xi)$ is dense in $\mathbb{P}_{<\gamma}(\xi)$ by the above, and clearly $\mathbb{P}_{\alpha}(\xi) \in \mathfrak{M}_{\gamma}$, we obtain $S \subseteq{ }^{\text {fin }} \bigvee \mathbb{P}_{\alpha}(\xi)$ for each tree $S \in \mathbf{Q}(\xi)=\mathbb{P}_{\gamma}(\xi)$, thus we have (2) of Definition 6.4.

Lemma 16.3 (in $\mathbf{L}$ ). Assume that, for each $n, \mathbf{D}_{n} \subseteq \square$ is open dense in $\square$, and let $\mathbf{T} \in \mathbb{\square}$. There is a multitree $\mathbf{S} \in \mathbb{\square}$, satisfying $\mathbf{S} \leqslant \mathbf{T}$ and $\mathbf{S} \subseteq{ }^{\mathrm{fd}} \bigvee \mathbf{D}_{n}$ for all $n$. Therefore $\mathbb{\square}$-generic extensions of $\mathbf{L}$ preserve $\omega_{1}^{\mathbf{L}}$.

Proof. There is a countable elementary submodel $M$ of $\left\langle\mathbf{L}_{\omega_{2}} ; \epsilon\right\rangle$, containing $\mathbf{T}$ and all sets $\mathbf{D}_{n}$. Then $M$ also contains $\omega_{1}$, as it is a definable set, and contains the sequence $\overrightarrow{\mathbb{P}}$ along with the derived sets $\mathbb{P}=\bigcup^{\mathrm{cw}} \overrightarrow{\mathbb{P}}, \mathbb{\nabla}=\mathbf{M T}(\mathbb{P})$, by the same reason. The set $M \cap \mathbf{L}_{\omega_{1}}$ is transitive. Indeed if $X \in M \cap \mathbf{L}_{\omega_{1}}$ then $X$ is at most countable, hence there exist functions $f: \omega \stackrel{\text { onto }}{\longrightarrow} X$. Let $f_{X}$ be the least of them in the sense of the Gödel wellordering $\leqslant_{\mathbf{L}}$ of $\mathbf{L}$. Then $f_{X} \in M$ since $X \in M$ and the ordering $\leqslant_{\mathbf{L}} \backslash \mathbf{L}_{\omega_{2}}$ is definable in $\mathbf{L}_{\omega_{2}}$. It follows that each $x \in X$ belongs to $M$ because $x=f_{X}(k)$ for some $k$.

Let $\phi: M \stackrel{\text { onto }}{\longrightarrow} \mathbf{L}_{\lambda}$ be the Mostowski collapse function, and $\alpha=\phi\left(\omega_{1}\right)$. Then $\alpha<\lambda<\omega_{1}$ and, by the transitivity, it holds $\left(^{*}\right) \phi(x)=x$ for all $x \in M \cap \mathbf{L}_{\omega_{1}}$. Thus $\phi(\xi)=\xi, \phi(T)=T, \phi(\mathbf{S})=\mathbf{S}$ for each ordinal $\xi \in M \cap \omega_{1}$, tree $T \in M \cap \mathbf{L T}$, multitree $\mathbf{S} \in M \cap \mathbf{M T}$. We conclude that $\phi(\overrightarrow{\mathbb{P}})=\overrightarrow{\mathbb{P}} \cap \mathbf{L}_{\alpha}=\overrightarrow{\mathbb{P}} \mid \alpha, \phi(\mathbb{P})=\mathbb{P}_{<\alpha}=$ $\bigcup_{\gamma<\alpha}^{\text {cw }} \mathbb{P}_{\alpha}$ (a multiforcing with $\left|\mathbb{P}_{<\alpha}\right|=\alpha$ ), and $\phi(\square)=\rrbracket \cap \mathbf{L}_{\alpha}=\mathbf{M T}\left(\mathbb{P}_{<\alpha}\right) \cap \mathbf{L}_{\alpha}$.

We assert that moreover $\phi(\square)=\mathfrak{S}_{\alpha}$, where, we recall, $\mathfrak{S}_{\alpha}=\mathfrak{S}\left(\mathbb{P}_{<\alpha}\right)$. Indeed by Definition 12.3, $\mathfrak{S}\left(\mathbb{P}_{<\alpha}\right)$ is equal to the closure of $\mathbf{M T}\left(\mathbb{P}_{<\alpha}\right) \cap \mathbf{L}_{\alpha}$ relatively to the three operations of Definition 9.1. But $\varphi\left(\mathbb{\square}=\mathbf{M T}\left(\mathbb{P}_{<\alpha}\right) \cap \mathbf{L}_{\alpha}\right.$, thus $\mathbf{M T}\left(\mathbb{P}_{<\alpha}\right) \cap \mathbf{L}_{\alpha}$ is already closed under the operations, since so is $\mathbb{\square}=\mathbf{M T}(\mathbb{P})$. We conclude that $\mathfrak{S}\left(\mathbb{P}_{<\alpha}\right)=\mathbf{M T}\left(\mathbb{P}_{<\alpha}\right) \cap \mathbf{L}_{\alpha}$.

Furthermore, a similar argument allows to prove that if $n<\omega$ then the set $\phi\left(\mathbf{D}_{n}\right)=\mathbf{D}_{n} \cap \mathbf{L}_{\alpha}=\mathbf{D}_{n} \cap \mathfrak{S}_{\alpha} \in \mathbf{L}_{\lambda}$ is open dense in $\mathfrak{S}\left(\mathbb{P}_{<\alpha}\right)$. In addition, $\phi(\mathbf{T})=\mathbf{T} \in \mathfrak{S}_{\alpha}$. On the other hand, by the elementarity, the ordinal $\alpha$ is uncountable in $\mathbf{L}_{\lambda}$. It follows that $\mathbf{L}_{\lambda} \subseteq \mathfrak{M}_{\alpha}$. However we have $\mathbb{P}_{<\alpha} \sqsubset_{\mathfrak{M}_{\alpha}} \mathbb{P}_{\alpha} \mid \alpha$ by $15.5(*)$, and also $\mathbf{T} \in \mathfrak{S}_{\alpha}=\mathfrak{S}\left(\mathbb{P}_{<\alpha}\right)$. Therefore, by Definition $12.3(\mathrm{C})$, there exists a multitree $\mathbf{S} \in \mathbf{M T}\left(\mathbb{P}_{\alpha}\right)$ satisfying $\mathbf{S} \leqslant \mathbf{T}$ and $\mathbf{S} \subseteq{ }^{\mathrm{fd}} \bigvee \phi\left(\mathbf{D}_{n}\right)$ for all $n$ Finally, $\mathbf{M T}\left(\mathbb{P}_{\alpha}\right) \subseteq \llbracket$ and $\phi\left(\mathbf{D}_{n}\right) \subseteq \mathbf{D}_{n}$. This ends the proof of the first claim.

To prove the second claim of the lemma, suppose towards the contrary that $\dot{f}$ is a name of a function from $\omega$ to $\omega_{1}^{\mathbf{L}}$, and some $\mathbf{T} \in \mathbb{\square}$ forces $\operatorname{ran} \dot{f}=\omega_{1}^{\mathbf{L}}$. Let $\mathbf{D}_{n \alpha}$ be the set of all multitrees $\mathbf{R} \in \mathbb{\square}$, either (1) incompatible with $\mathbf{T}$ in

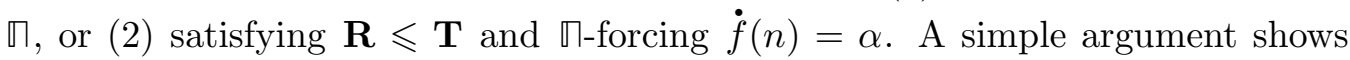
that every set $\mathbf{D}_{n}=\bigcup_{\alpha} \mathbf{D}_{n \alpha}$ is dense in $\square$. By the first claim of the lemma, there exists a multitree $\mathbf{S} \in \mathbb{\square}$ satisfying $\mathbf{S} \leqslant \mathbf{T}$ and $\mathbf{S} \subseteq{ }^{\mathrm{fd}} \bigvee \mathbf{D}_{n}, \forall n$. Let the relations 
$\mathbf{S} \subseteq{ }^{\text {fd }} \bigvee \mathbf{D}_{n}$ be witnessed by finite sets $\mathbf{D}_{n}^{\prime} \subseteq \mathbf{D}_{n}$. Accordingly, the sets $A_{n}=\{\alpha$ : $\left.\mathbf{D}_{n}^{\prime} \cap \mathbf{D}_{n \alpha} \neq \varnothing\right\}$ are finite, hence the union $A=\bigcup_{n} A_{n}$ is countable in $\mathbf{L}$, i.e., $\omega_{1}^{\mathbf{L}} \nsubseteq \nsubseteq A$. On the other hand, we assert that $\mathbf{S}$ forces $\dot{f}(n) \in A_{n}$, for each $n$. This implies a contradiction and accomplishes the proof.

To finally prove that $\mathbf{S}$ forces $\dot{f}(n) \in A_{n}$, suppose to the contrary that $\mathbf{R} \in \mathbb{\square}$, $\mathbf{R} \leqslant \mathbf{S}$, and $\mathbf{R}$ forces $\dot{f}(n)=\alpha$, where $\alpha<\omega_{1}^{\mathbf{L}}, \alpha \notin A_{n}$. Then $\mathbf{R} \subseteq{ }^{\text {fd }} \vee \mathbf{D}_{n}$ by means of the same finite set $\mathbf{D}_{n}^{\prime} \subseteq \mathbf{D}_{n}$. Lemma 7.4(v) provides a string $\sigma \in 2^{<\omega}$ and a multitree $\mathbf{U} \in \mathbf{D}_{n}^{\prime}$ such that $\mathbf{R}^{\prime}=\mathbf{R}(\Rightarrow \sigma) \leqslant \mathbf{U}$. Note that $\mathbf{R}^{\prime} \in \mathbb{\square}$ by Lemma 9.3(i). Thus the multitrees $\mathbf{R}$ and $\mathbf{U}$ are compatible in $\mathbb{\square}$. Finally $\mathbf{U} \in \mathbf{D}_{n}^{\prime} \subseteq \mathbf{D}_{n}$, therefore $\mathbf{U} \in \mathbf{D}_{n \gamma}$ for some $\gamma$. Then by definition $\mathbf{U}$ forces $\dot{f}(n)=\gamma$, where $\gamma \in A_{n}$, that is, $\gamma \neq \alpha$. However $\mathbf{R}$ forces $\dot{f}(n)=\alpha$, where $\alpha \notin A_{n}$, which is a contradiction.

Lemma 16.4 (in $\mathbf{L}$ ). If a set of multitrees $Q \subseteq \mathbf{M T}$ belongs to $\Sigma_{\mathrm{n}-2}^{\mathrm{HC}}(\mathrm{HC})$ and $Q^{-}=\{\mathbf{T} \in \mathbf{M T}: \neg \exists \mathbf{S} \in Q(S \leqslant T)\}$, then the set $\square \cap\left(Q \cup Q^{-}\right)$is dense in $\square$. In particular if $Q$ is dense in $\mathbf{M T}$ then $Q \cap \mathbb{Q}$ is dense in $\square$.

Proof. Consider a multitree $\mathbf{T}_{0} \in \mathbb{\square}=\mathbf{M T}(\mathbb{P})$, thus $\mathbf{T}_{0} \in \mathbf{M T}\left(\mathbb{P}_{<\alpha_{0}}\right), \alpha_{0}<\omega_{1}$. The set $\Delta$ of all sequences $\overrightarrow{\mathbf{P}} \in \overrightarrow{\mathbf{M F}}$, such that $\overrightarrow{\mathbb{P}} \mid \alpha_{0} \subseteq \overrightarrow{\mathbf{P}}$ and $\exists \mathbf{T} \in Q \cap$ $\left(\mathbf{M T}\left(\bigcup^{\mathrm{cw}} \overrightarrow{\mathbf{P}}\right)\right)\left(\mathbf{T} \leqslant \mathbf{T}_{0}\right)$, belongs to $\Sigma_{\mathfrak{n}-2}^{\mathrm{HC}}(\mathrm{HC})$ as so does $Q$. Therefore there is an ordinal $\alpha<\omega_{1}$ such that the sequence $\overrightarrow{\mathbb{P}}\lceil\alpha$ blocks $\Delta$.

Case 1: $\overrightarrow{\mathbb{P}}\left\lceil\alpha \in \Delta\right.$; let this be witnessed by $\mathbf{T} \in Q \cap\left(\mathbf{M T}\left(\bigcup^{\mathrm{cw}}(\overrightarrow{\mathbb{P}}\lceil\alpha))\right)\right.$. Then $\alpha_{0} \leq \alpha$ and the multitree $\mathbf{T}$ belongs to $Q \cap \mathbb{Q}$ and satisfies $\mathbf{T} \leqslant \mathbf{T}_{0}$.

Case 2: no sequence in $\Delta$ extends $\overrightarrow{\mathbb{P}}\left\lceil\alpha\right.$. Let $\gamma=\max \left\{\alpha, \alpha_{0}\right\}$. Then $\mathbb{P}_{<\gamma} \sqsubset_{\mathfrak{M}_{\gamma}}$ $\mathbb{P}_{\gamma}\left\lceil\gamma\right.$ by $15.5(*)$. As $\alpha_{0} \leq \gamma$, there exists a multitree $\mathbf{T} \in \mathbf{M T}\left(\mathbb{P}_{\gamma}\right), \mathbf{T} \leqslant \mathbf{T}_{0}$. We can wlog assume that $|\mathbf{T}|=\left|\mathbb{P}_{\gamma}\right|$, that is $=\gamma+1$. Then $\mathbf{T}(\xi) \in \mathbb{P}_{\gamma}(\xi)$ for all $\xi \leq \gamma$. It remains to prove that $\mathbf{T} \in Q^{-}$.

Suppose to the contrary that $\mathbf{T} \notin Q^{-}$. By definition there is a multitree $\mathbf{S} \in Q$, $\mathbf{S} \leqslant \mathbf{T}$. Then $\gamma+1=|\mathbf{T}| \subseteq|\mathbf{S}|$. We can assume that $|\mathbf{S}|=\lambda<\omega_{1}, \lambda \geq \gamma+1$. We are going to define a sequence $\overrightarrow{\mathbf{P}}=\left\langle\mathbf{P}_{\alpha}\right\rangle_{\alpha<\lambda} \in \overrightarrow{\mathbf{M F}}$, which extends $\overrightarrow{\mathbb{P}} \mid \gamma$, that is, $\mathbf{P}_{\alpha}=\mathbb{P}_{\alpha}$ for all $\alpha<\gamma$, and satisfies $\mathbf{S} \in \mathbf{M T}\left(\bigcup^{\mathrm{cw}} \overrightarrow{\mathbf{P}}\right)$. This implies $\overrightarrow{\mathbf{P}} \in \Delta$ by the choice of $\mathbf{S}$, which contradicts to the Case 2 hypothesis and completes the proof of $\mathbf{T} \in Q^{-}$and the proof of the lemma.

Thus we have to appropriately define multiforcings $\mathbf{P}_{\alpha}, \gamma \leq \alpha<\lambda$. We begin with $\mathbf{P}_{\gamma}$. This is based on the multiforcing $\mathbb{P}_{\gamma}$. Note that $\mathbf{S}(\xi) \subseteq \mathbf{T}(\xi) \in \mathbb{P}_{\gamma}(\xi)$ for all $\xi \leq \gamma$. We put $\mathbf{P}_{\gamma}(\xi)=\mathbb{P}_{\gamma}(\xi) \cup\left\{\sigma \cdot(\mathbf{S}(\xi)(\rightarrow t)): t, \sigma \in 2^{<\omega}\right\}$ for all $\xi \leq \gamma$. Every "new" tree $S=\sigma \cdot(\mathbf{S}(\xi)(\rightarrow t))$ satisfies $S \subseteq \sigma \cdot \mathbf{T}(\xi)$, where $\sigma \cdot \mathbf{T}(\xi) \in \mathbb{P}_{\gamma}(\xi)$. However $\mathbb{P}_{<\gamma} \sqsubset_{\mathfrak{M}_{\gamma}}^{+} \mathbb{P}_{\gamma}$ by Definition 15.5(*). Therefore $\mathbb{P}_{<\gamma} \sqsubset_{\mathfrak{M}_{\gamma}}^{+} \mathbf{P}_{\gamma}$ as well. Thus the term $\mathbf{P}_{\gamma}$ extends the system $\overrightarrow{\mathbb{P}}\left\lceil\gamma=\left\langle\mathbb{P}_{\alpha}\right\rangle_{\alpha<\gamma}=\left\langle\mathbf{P}_{\alpha}\right\rangle_{\alpha<\gamma} \in \overrightarrow{\mathbf{M F}}_{\gamma}\right.$ to a system in $\overrightarrow{\mathbf{M F}}_{\gamma+1}$, and we have $\mathbf{S}(\xi) \in \mathbf{P}_{\gamma}(\xi)$ for all $\xi \leq \gamma$. The extended system can be further extended to a system in $\overrightarrow{\mathbf{M F}}_{\lambda}$ by terms $\mathbf{P}_{\alpha}, \gamma<\alpha<\lambda$, by induction as in the proof of 15.2(ii), with the amendment that $\mathbf{P}_{\alpha}(\alpha)=P_{\text {coh }} \cup\{\sigma \cdot(\mathbf{S}(\alpha)(\rightarrow t))$ : $\left.t, \sigma \in 2^{<\omega}\right\}$, rather than just $\mathbf{P}_{\alpha}(\alpha)=P_{\text {coh }}$, for all $\alpha$. 


\section{Generic extension}

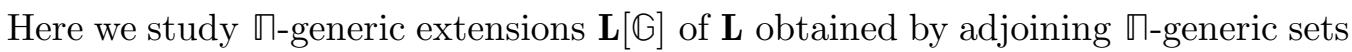
$\mathbb{G} \subseteq \square$ to $\mathbf{L}$. We will use the forcing notion $\square=\mathbf{M T}(\mathbb{P}) \in \mathbf{L}$ and other notation of Definition 15.5, with the difference that the reasoning will not be relativized to $\mathbf{L}$ by default, and accordingly the first uncountable cardinal in $\mathbf{L}$ will be denoted by $\omega_{1}^{\mathbf{L}}$ instead of $\omega_{1}$.

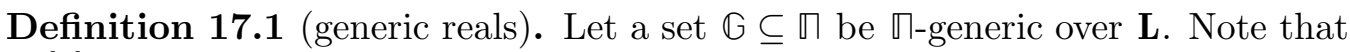
$\omega_{1}^{\mathbf{L}[\mathbb{G}]}=\omega_{1}^{\mathbf{L}}$ by Lemma 16.3 .

If $\xi<\omega_{1}^{\mathbf{L}}$ then $\mathbb{G}(\xi)=\{\mathbf{T}(\xi): \xi \in|\mathbf{T}| \wedge \mathbf{T} \in \mathbb{G}\}$ is a set $\mathbb{P}(\xi)$-generic over $\mathbf{L}$, the intersection $X_{\xi}=\bigcap_{T \in \mathbb{G}(\xi)}[T]$ contains a single real $\boldsymbol{x}_{\xi}=\boldsymbol{x}_{\xi}[\mathbb{G}] \in 2^{\omega}$, and this real is $\mathbb{P}(\xi)$-generic over $\mathbf{L}$. These reals are assembled into a "multireal" $\boldsymbol{x}[\mathbb{G}]=\left\langle\boldsymbol{x}_{\xi}[\mathbb{G}]\right\rangle_{\xi<\omega_{1}^{\mathbf{L}}} \in\left(2^{\omega}\right)^{\omega_{1}^{\mathbf{L}}}$.

Corollary $\mathbf{1 7 . 2}$ (of 16.1 and the product forcing theorem). If $B \in \mathbf{L}, B \subseteq \omega_{1}^{\mathbf{L}}$

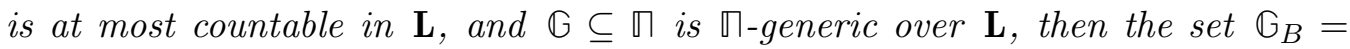

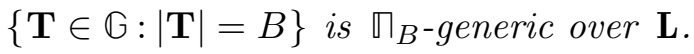

Recall that $C_{\neq \xi}=\omega_{1}^{\mathbf{L}} \backslash\{\xi\}$.

Proposition $\mathbf{1 7 . 3}$ (in terms of Definition 17.1). If $\xi<\omega_{1}^{\mathbf{L}}$ then the real $\boldsymbol{x}_{\xi}[\mathbb{G}]$ is not $\left(\left\{\mathbb{G} \mid C_{\neq \xi}\right\} \cup\right.$ Ord)-definable in $\mathbf{L}[\mathbb{G}]$, in particular, $\boldsymbol{x}_{\xi}[\mathbb{G}] \notin \mathbf{L}\left[\mathbb{G} \mid C_{\neq \xi}\right]$.

Proof. See the proof of Lemma 14.5 in [16], based on the product forcing theorem and the $\mathrm{E}_{0}$-invariance of each component $\mathbb{P}(\xi)$ in the sense of $6.1(\mathrm{~B})$.

The next theorem belongs to the type of "continuous reading of names" theorems in theory of forcing extensions. It involves the coding of continuous maps by Definition 12.1, and asserts that reals $x \in 2^{\omega}$ in $\mathbb{~}$-generic extensions are obtained by applications of continuous maps coded in $\mathbf{L}$ to suitable sequences of generic reals. To render the notation less cumbersome, if $\mathbf{c} \in \mathbf{L}$ and $\mathbf{c} \in \mathrm{CCF}$ in $\mathbf{L}$, and $\mathbb{G} \subseteq \mathbb{\square}$ is generic over $\mathbf{L}$, then we put $f^{\mathbf{c}}[\mathbb{G}]:=f^{\mathbf{c}}(\boldsymbol{x}[G] \mid B)$, where $B=|\mathbf{c}|$.

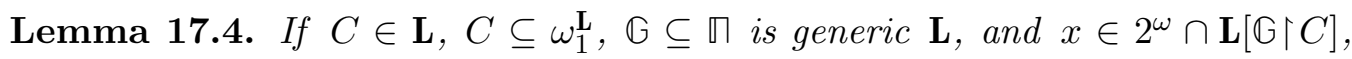
then there is a code $\mathbf{c} \in \mathrm{CCF} \cap \mathbf{L}$, such that $|\mathbf{c}| \subseteq C$ and $x=f^{\mathbf{c}}[\mathbb{G}]$.

Proof. Let $\dot{\boldsymbol{x}}$ be a name for $x$ in the forcing language related to the forcing notion ॠ. Thus the indexed family of sets

$$
A_{k i}=\{\mathbf{T} \in \mathbb{\square}: \mathbf{T} \text { forces that } \dot{\boldsymbol{x}}(k)=i\}, \quad k<\omega, \quad i=0,1,
$$

belongs to $\mathbf{L}$ and we have (A) $x(k)=i \Longleftrightarrow \mathbb{G} \cap A_{k i} \neq \varnothing$, (B) $A_{k 0} \cap A_{k 1}=\varnothing$, and (C) each set $A_{k}=A_{k 0} \cup A_{k 1}$ is open dense in $\square$. We can assume that $\dot{\boldsymbol{x}}$ contains an explicit effective construction of $x$ from $\mathbb{G}\left\lceil C\right.$, and then: (*) if $\mathbf{S} \in A_{k i}$ then $\mathbf{S} \uparrow(C \cap|\mathbf{S}|) \in A_{k i}$ as well. 
The set $D=\left\{\mathbf{T} \in \mathbb{T}: \forall k\left(\mathbf{T} \subseteq{ }^{\mathrm{fd}} \bigvee A_{k}\right)\right\}$ also is dense in $\mathbb{\square}$ by Lemma 16.3. Therefore, by the genericity, there is a multitree $\mathbf{T}^{\prime} \in \mathbb{G}$, such that $\mathbf{T}^{\prime} \subseteq{ }^{\mathrm{fd}} \bigvee A_{k}$ for all $k$. In addition, $\left(^{*}\right)$ implies that the multitree $\mathbf{T}=\mathbf{T}^{\prime} \uparrow(C \cap|\mathbf{T}|) \in \mathbb{G}$ also satisfies $\mathbf{T} \subseteq{ }^{\mathrm{fd}} \bigvee A_{k}, \forall k$, but now $|\mathbf{T}| \subseteq C$.

This means (Definition 7.2) that, in $\mathbf{L}$, there exists a sequence of finite sets $F_{k} \subseteq A_{k}$ which witness $\mathbf{T} \subseteq{ }^{\mathrm{fd}} \bigvee A_{k}$ in the sense that: 1) $|\mathbf{U}| \subseteq B=|\mathbf{T}|$ for all $\left.\mathbf{U} \in F_{k}, 2\right)[\mathbf{T}] \subseteq \bigcup_{\mathbf{U} \in F_{k}}[\mathbf{U} \uparrow B]$, and 3$)[\mathbf{U} \uparrow B] \cap[\mathbf{V} \uparrow B]=\varnothing$ for all $\mathbf{V} \neq \mathbf{U}$ in $F_{k}$. We put $F_{k i}=F_{k} \cap A_{k i}, i=0,1$.

Now arguing in $\mathbf{L}$ we define a continuous $f:[\mathbf{T}] \rightarrow 2^{\omega}$ as follows: $f(y)(k)=i$, if there is a multitree $\mathbf{S} \in F_{k i}$ with $y\left\lceil|\mathbf{S}| \in[\mathbf{S}]\right.$. Then $f=f^{\mathbf{c}} \uparrow[\mathbf{S}]$ by Lemma 12.2, where $\mathbf{c}$ is a suitable code $\mathrm{CCF}_{B} \cap \mathbf{L}$. One easily verifies that $x=f^{\mathbf{c}}[\mathbb{G}]$

By the next theorem, the relation $y \notin \mathbf{L}[x]$ between reals $x, y \in 2^{\omega}$ in $\mathbf{L}[\mathbb{G}]$ is fully determined by a generic real $\boldsymbol{x}_{\xi}[\mathbb{G}]$, so that $\boldsymbol{x}_{\xi}[\mathbb{G}]$ belongs to $\mathbf{L}[y]$ but $x$ belongs to $\mathbf{L}\left[\boldsymbol{x}[\mathbb{G}] \mid C_{\neq \xi}\right]$, where definitely $\boldsymbol{x}_{\xi}[\mathbb{G}] \notin \mathbf{L}\left[\boldsymbol{x}[\mathbb{G}] \mid C_{\neq \xi}\right]$ by Proposition 17.3.

Theorem 17.5. If a set $\mathbb{G} \subseteq \mathbb{\square}$ is generic over $\mathbf{L}, x, y \in 2^{\omega} \cap \mathbf{L}[\mathbb{G}]$, and $y \notin \mathbf{L}[x]$, then there is an ordinal $\xi<\omega_{1}^{\mathbf{L}}$ such that $x \in \mathbf{L}\left[\boldsymbol{x}[\mathbb{G}]\left\lceil C_{\neq \xi}\right]\right.$ but $\boldsymbol{x}_{\xi}[\mathbb{G}] \in \mathbf{L}[y]$, and in addition, $\boldsymbol{x}_{\xi}[\mathbb{G}]=g(y)$, where $g: 2^{\omega} \rightarrow 2^{\omega}$ is a continuous map coded in $\mathbf{L}$.

Proof. By Lemma 17.4, there exist codes $\mathbf{c}, \mathbf{d} \in \mathrm{CCF} \cap \mathbf{L}$, such that $x=f^{\mathbf{c}}[\mathbb{G}]$ and $y=f^{\mathbf{d}}[\mathbb{G}$. Let $B=|\mathbf{c}| \cup|\mathbf{d}|$; we can assume that $|\mathbf{c}|=|\mathbf{d}|=B$.

$W e$ argue in $\mathbf{L}$. The set $\mathbf{D}$ of all multitrees $\mathbf{S} \in \mathbf{M T}_{B}$ such that either (i) $f^{\mathbf{d}}$ is reduced to $f^{\mathbf{c}}$ on $[\mathbf{S}]$, or (ii) $f^{\mathbf{d}}$ captures some ordinal $\xi \in B$ on $[\mathbf{S}]$ and $f^{\mathbf{c}}$ is reduced to the set $B \backslash\{\xi\}$ on $[\mathbf{S}]$, is dense in $\mathbf{M T}_{B}$ by Theorem 8.2. It follows by Lemma 16.4 that the set $\mathbf{D}^{\prime}=\mathbf{D} \cap \square_{B}$ is dense in $\square_{B}=\{\mathbf{R} \in \mathbb{\square}:|\mathbf{R}|=B\}$.

We argue in $\mathbf{L}[\mathbb{G}]$. We have $\mathbb{G} \cap \mathbf{D} \neq \varnothing$ by Corollary 17.2. Let $\mathbf{S} \in \mathbb{G} \cap \mathbf{D}$; then $\boldsymbol{x}[\mathbb{G}]\left\lceil B \in[\mathbf{S}]\right.$. Note that (i) fails for this $\mathbf{S}$, since (i) implies $f^{\mathbf{d}}(z)=g\left(f^{\mathbf{c}}(z)\right)$ for all $z \in[\mathbf{S}]$, where $g: 2^{\omega} \rightarrow 2^{\omega}$ is a continuous map coded in $\mathbf{L}$, thus (with $z=\boldsymbol{x}[\mathbb{G}]\lceil B$ ) we get $y=g(x)$, and further $y \in \mathbf{L}[x]$ (as $g$ is coded in $\mathbf{L}$ ), a contradiction to the condition of the theorem. Thus (ii) holds, i.e., still in $\mathbf{L}, f^{\mathbf{d}}$ captures an ordinal $\xi \in B$ on $[\mathbf{S}]$, while $f^{\mathbf{c}}$ is reduced to $B \backslash\{\xi\}$ on $[\mathbf{S}]$.

By the compactness of the spaces considered, this implies the existence of continuous maps $f:\left(2^{\omega}\right)^{B \backslash\{\xi\}} \rightarrow 2^{\omega}$ and $g: 2^{\omega} \rightarrow 2^{\omega}$, both coded in $\mathbf{L}$ and satisfying $f^{\mathbf{c}}(z)=f\left(z\lceil(B \backslash\{\xi\}))\right.$ and $z(\xi)=g\left(f^{\mathbf{d}}(z)\right)$ for all $z \in \mathbf{S}$. In particular, for $z=\boldsymbol{x}[\mathbb{G}]\left\lceil B\right.$, we have $x=f^{\mathbf{c}}(\boldsymbol{x}[\mathbb{G}] \uparrow(B \backslash\{\xi\}))$, hence $x \in \mathbf{L}\left[\boldsymbol{x}[\mathbb{G}]\left\lceil C_{\neq \xi}\right]\right.$, and $\boldsymbol{x}_{\xi}[\mathbb{G}]=g(y)$, hence $\boldsymbol{x}_{\xi}[\mathbb{G}] \in \mathbf{L}[y]$.

\section{Definability of generic reals}

We continue to argue in terms of definitions 15.5 and 17.1. Now the main goal

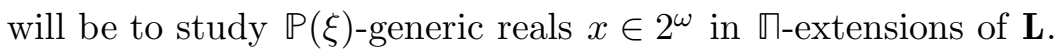

Theorem 18.1. In any $\square$-generic extension $\mathbf{L}[\mathbb{G}]$ of $\mathbf{L}$, it is true that: if $\xi<\omega_{1}^{\mathbf{L}}$ then the set $X_{\xi}=\left[\boldsymbol{x}_{\xi}[\mathbb{G}]\right]_{\mathrm{E}_{0}}=\left\{\sigma \cdot \boldsymbol{x}_{\xi}[\mathbb{G}]: \sigma \in 2^{<\omega}\right\}$ is equal to the set $Y_{\xi}=$ $\bigcap_{\xi \leq \alpha<\omega_{1}^{\mathrm{L}}} \bigcup_{U \in \mathbb{P}_{\alpha}(\xi)}[U]$. 
Proof. The real $x=\boldsymbol{x}_{\xi}[\mathbb{G}] \in 2^{\omega}$ is $\mathbb{P}(\xi)$-generic, while every set of the form $\mathbb{P}_{\alpha}(\xi)$ is pre-dense in $\mathbb{P}(\xi)$ by Lemma 16.2. Therefore $x \in Y_{\xi}$. Moreover all sets $\mathbb{P}_{\alpha}(\xi)$ are $\mathbf{L T}$-forcings by construction, hence they are $\mathrm{E}_{0}$-invariant in the sense of $6.1(\mathrm{~B})$. It follows that $X_{\xi} \subseteq Y_{\xi}$.

To establish the inverse, assume that $y_{0} \in Y_{\xi}$ in $\mathbf{L}[\mathbb{G}]$. By Lemma 17.4, there is a code $\mathbf{c} \in \mathrm{CCF} \cap \mathbf{L}$ such that $y_{0}=f^{\mathbf{c}}[\mathbb{G}]=f^{\mathbf{c}}(\boldsymbol{x}[\mathbb{G}]\lceil B)$, where $B=|\mathbf{c}|$. Consider the set $\mathbf{D}$ of all multitrees $\mathbf{S} \in \mathbb{\square}_{B}$ such that either (i) there is a string $\sigma \in 2^{<\omega}$ such that $f^{\mathbf{c}}(x)=\sigma \cdot x(\xi)$ for all $x \in[\mathbf{S}]$, or (ii) there exists an ordinal $\alpha, \xi \leq \alpha<\omega_{1}$, such that $f^{\mathbf{c}}(x) \notin \bigcup_{U \in \mathbb{P}_{\alpha}(\xi)}[U]$ for all $x \in[\mathbf{S}]$.

Lemma 18.2. The set $\mathbf{D}$ is dense in $\square_{B}$.

Proof. Let $\mathbf{T} \in \mathbb{\square}_{B}$, then $|\mathbf{T}|=B$. There is an ordinal $\alpha<\omega_{1}^{\mathbf{L}}$ such that 1) $B \subseteq \alpha$ - hence $\xi<\alpha, 2) \mathbf{T} \in \mathfrak{S}_{\alpha}=\mathfrak{S}\left(\mathbb{P}_{<\alpha}\right)$, and 3) $\mathbf{c} \in \mathfrak{M}_{\alpha}$. Note that $\mathbb{P}_{<\alpha} \sqsubset_{\mathfrak{M}_{\alpha}}^{+} \mathbb{P}_{\alpha}$ holds by $15.5(*)$. Therefore by Definition 12.3(D) there is a multitree $\mathbf{S} \in \mathbf{M T}\left(\mathbb{P}_{\alpha}\right)$ such that $|\mathbf{S}|=|\mathbf{T}|=B, \mathbf{S} \leqslant \mathbf{T}$, and either (i) there is a string $\sigma \in 2^{<\omega}$ satisfying $f^{\mathbf{c}}(x)=\sigma \cdot x(\xi)$ for all $x \in[\mathbf{S}]$, or (ii) $f^{\mathbf{c}}(x) \notin \bigcup_{U \in \mathbb{P}_{\alpha}(\xi)}[U]$ for all $x \in[\mathbf{S}]$. Thus $\mathbf{S} \in \mathbf{D}$, getting the density.

$\square$ (Lemma)

We return to the theorem. Corollary 17.2 implies $\mathbb{G} \cap \mathbf{D} \neq \varnothing$ by the lemma. Let $\mathbf{S} \in \mathbb{G} \cap \mathbf{D}$. In particular $x_{0}=\boldsymbol{x}[\mathbb{G}] \uparrow B \in[\mathbf{S}]$. It follows that $\mathbf{S}$ does not satisfy (ii) of the definition of $\mathbf{D}$, since $y_{0}=f^{\mathbf{c}}\left(x_{0}\right) \in Y_{\xi}$. Therefore $\mathbf{S}$ satisfies (i) of the definition of $\mathbf{D}$ with some $\sigma \in 2^{<\omega}$. Then $y_{0}=f^{\mathbf{c}}\left(x_{0}\right)=\sigma \cdot x_{0}(\xi)=\sigma \cdot \boldsymbol{x}[\mathbb{G}](\xi)=$ $\sigma \cdot \boldsymbol{x}_{\xi}[\mathbb{G}]$, that is, $y_{0} \in X$, as required.

One easily proves that, under the conditions of the theorem, the set $X_{\xi}=Y_{\xi}$ is equal to the set of all $\mathbb{P}(\xi)$-generic reals $y \in 2^{\omega}$, see [13].

\section{Non-uniformizable set}

Here we prove claim (i) of Theorem 15.6. To begin with, we define a nonuniformizable set in the "rectangle" $\omega_{1}^{\mathbf{L}} \times 2^{\omega}$.

Lemma 19.1. Under the conditions of Theorem 15.6, the set $K=\{\langle\xi, x\rangle$ : $\left.\xi<\omega_{1}^{\mathbf{L}} \wedge x \in\left[\boldsymbol{x}_{\xi}[\mathbb{G}]\right]_{\mathrm{E}_{0}}\right\}$ belongs to $\mathbf{L}[\mathbb{G}]$ and has the following properties in $\mathbf{L}[\mathbb{G}]$ :

(i) $K$ belongs to the definability class $\Pi_{\mathrm{m}-1}^{\mathrm{HC}}$;

(ii) if $\xi<\omega_{1}$ then the cross-section $K_{\xi}=\{x:\langle\xi, x\rangle \in K\}$ is a $\mathrm{E}_{0}$-class;

(iii) the set $K$ is not ROD-uniformizable.

Proof. (ii) is quite obvious: $K_{\xi}=\left[x_{\xi}[\mathbb{G}]\right]_{\mathrm{E}_{0}}$. To prove (i) we note that Lemma16.3 implies $\omega_{1}=\omega_{1}^{\mathbf{L}}$ in $\mathbf{L}[\mathbb{G}]$. Therefore by Theorem 18.1, the sentence $\langle\xi, x\rangle \in K$ is equivalent to

$$
\xi<\omega_{1} \wedge \forall \alpha\left(\xi \leq \alpha<\omega_{1} \Longrightarrow \exists T \in \mathbb{P}_{\alpha}(\xi)(x \in[T])\right)
$$


Yet the formula in the outer brackets here expresses a $\Pi_{\mathrm{m}-1}^{\mathrm{HC}}$ relation by condition (i) of Theorem 15.4. (The quantifier $\exists T \in \mathbb{P}_{\alpha}(\xi)$ is bounded, hence it does not affect the definability estimation.)

To prove (iii) suppose towards the contrary that it is true in $\mathbf{L}[\mathbb{G}]$ that $R \subseteq K$ is an uniformizing ROD set. Let $r \in 2^{\omega} \cap \mathbf{L}[\mathbb{G}]$ be a real which witnesses that $R$ is $\{r\} \cup$ Ord-definable in $\mathbf{L}[\mathbb{G}]$. Lemma 16.3 (preservation of $\omega_{1}^{\mathbf{L}}$ ) implies the existence of an ordinal $\xi<\omega_{1}^{\mathbf{L}}$ such that $r \in \mathbf{L}[\mathbb{G} \uparrow\{\eta: \eta<\xi\}]$, hence $r \in \mathbf{L}\left[\mathbb{G} \mid C_{\neq \xi}\right]$, where $C_{\neq \xi}=\omega_{1}^{\mathbf{L}} \backslash\{\xi\}$. Therefore the unique real $x \in 2^{\omega}$, satisfying $\langle\xi, x\rangle \in R$, is $\left(\left\{\mathbb{G} \mid C_{\neq \xi}\right\} \cup\right.$ Ord)-definable in $\mathbf{L}[\mathbb{G}]$. However $R \subseteq K$, thus $x \mathrm{E}_{0} \boldsymbol{x}_{\xi}[\mathbb{G}]$. It follows that the generic real $\boldsymbol{x}_{\xi}[\mathbb{G}]$ itself is $\left(\left\{\mathbb{G} \mid C_{\neq \xi}\right\} \cup \mathbf{O r d}\right)$-definable in $\mathbf{L}[\mathbb{G}]$. But this contradicts to Proposition 17.3.

To transform the set $K=K[\mathbb{G}]$ into a similar non-uniformizable set in the plane $2^{\omega} \times 2^{\omega}$, we make use of the following rather elementary transformation.

Let $\mathbb{Q}=\left\{q_{n}: n<\omega\right\}$ be a recursive enumeration of the rationals. If $z \in 2^{\omega}$ then let $Q_{z}=\left\{q_{n}: z(n)=1\right\} \subseteq \mathbb{Q}, Q_{z}^{\prime} \subseteq Q_{z}$ be the largest (perhaps, empty) well-ordered initial segment of $Q_{z}$, and let $|z|<\omega_{1}$ be the ordinal number of $Q_{z}^{\prime}$; thus obviously $\left\{|z|: z \in 2^{\omega}\right\}=\omega_{1}$.

Lemma 19.2. Under the conditions of Theorem 15.6, the set

$$
W=\left\{\langle z, x\rangle \in 2^{\omega} \times 2^{\omega}:\langle|z|, x\rangle \in K\right\}
$$

belongs to $\mathbf{L}[\mathbb{G}]$ and has the following properties in $\mathbf{L}[\mathbb{G}]$ :

(i) $W$ belongs to the definability class $\Pi_{\mathrm{m}}^{1}$;

(ii) if $z \in 2^{\omega}$ then the cross-section $W_{z}=\{x:\langle z, x\rangle \in W\}$ is a $\mathrm{E}_{0}$-class;

(iii) the set $W$ is not ROD-uniformizable.

Proof. The set $W$ belongs to $\Pi_{\mathrm{n}-1}^{\mathrm{HC}}$ since so does $K$; indeed the map $z \mapsto|z|$ is $\Delta_{1}^{\mathrm{HC}}$. Therefore by the transfer theorem (see e.g. 9.1 in [17]), $W$ is a $\Pi_{\mathrm{m}}^{1}$ set.

Further, each cross-section $W_{z}$ coincides with the corresponding cross-section $K_{\xi}$ of $K$, where $\xi=|z|$, thus $W_{z}$ is a $\mathrm{E}_{0}$-class.

To prove (iii), suppose to the contrary that $W$ is uniformized by a ROD set $S \subseteq W$. As $\omega_{1}^{\mathbf{L}}=\omega_{1}$ holds, for every ordinal $\xi<\omega_{1}$ there is a real $z \in 2^{\omega} \cap \mathbf{L}$

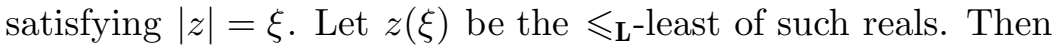

$$
R=\{\langle\xi, x\rangle \in K:\langle z(\xi), x\rangle \in S\}
$$

is a ROD subset of $K$, which uniformizes the set $K$, contrary to Lemma 19.1. Thus $W$ satisfies (i), (ii), (iii).

Proof (Theorem 15.6(i)). Obvious by Lemma 19.2. 


\section{Auxiliary forcing relation}

Here we define a key instrumentarium for the proof of (ii) of Theorem 15.6. This is a forcing-type relation forc. It is not directly connected with the forcing notion $\mathbb{P}$, but rather related to the countable-support product $\mathbf{L T}^{\omega_{1}}$. But it happens to be compatible with the $\square$-forcing relation for formulas of certain quantifier complexity (Lemma 21.2). An important property of forc will be its permutation-invariance (Lemma 21.3), a property which the $\mathbb{P}$-forcing relation definitely lacks. This will be the key argument in the proof of Theorem 22.1.

\section{We argue in $\mathbf{L}$.}

Let $\mathscr{L}$ be a language containing variables $i, j, k, \ldots$ of type 0 with the domain $\omega$, and variables $x, y, z, \ldots$ of type 1 with the domain $2^{\omega}$. Let terms be variables of type 0 and expressions of the form $x(k)$. Atomic formulas are those of the form $R\left(t_{1}, \ldots, t_{n}\right)$, where $R \subseteq \omega^{n}$ is any $n$-ary relation on $\omega$ in $\mathbf{L}$. Formulas containing no quantifiers over type 1 variables, are arithmetic. Formulas of the form

$$
\exists x_{1} \forall x_{2} \exists x_{3} \ldots \exists(\forall) x_{n} \Psi \quad \text { and } \quad \forall x_{1} \exists x_{2} \forall x_{3} \ldots \forall(\exists) x_{n} \Psi,
$$

where $\Psi$ is arithmetic, belong to types resp. $\mathscr{L} \Sigma_{n}^{1}, \mathscr{L} \Pi_{n}^{1}$.

Additionally, we allow codes $\mathbf{c} \in \mathrm{CCF}$ to substitute free variables of type 1 . We let $|\varphi|=\bigcup_{\mathbf{c} \in \varphi}|\mathbf{c}|$ for any $\mathscr{L}$-formula, where $\mathbf{c} \in \varphi$ means that a code $\mathbf{c}$ occurs in $\varphi$. The semantics is as follows. Let $\varphi:=\varphi\left(\mathbf{c}_{1}, \ldots, \mathbf{c}_{k}\right)$ be a $\mathscr{L}$-formula, and all codes in CCF occurring in $\varphi$ are explicitly indicated, and $|\varphi| \subseteq B \subseteq \omega_{1}$. If $x \in\left(2^{\omega}\right)^{B}$ then let $\varphi[x]$ denote the formula $\varphi\left(f^{\mathbf{c}_{1}}\left(x \uparrow\left|f^{\mathbf{c}_{1}}\right|\right), \ldots, f^{\mathbf{c}_{k}}\left(x \uparrow\left|f^{\mathbf{c}_{k}}\right|\right)\right)$; all elements $f^{\mathbf{c}_{i}}\left(x \uparrow\left|f^{\mathbf{c}_{i}}\right|\right)$ are reals in $2^{\omega}$.

Arithmetic formulas and those in $\mathscr{L} \Sigma_{n}^{1} \cup \mathscr{L} \Pi_{n}^{1}, n \geq 1$, will be called normal. If $\varphi$ is a formula in $\mathscr{L} \Sigma_{n}^{1}$ or $\mathscr{L} \Pi_{n}^{1}$ then $\varphi^{-}$is the result of canonical transformation of $\neg \varphi$ to resp. $\mathscr{L} \Pi_{n}^{1}, \mathscr{L} \Sigma_{n}^{1}$ form. We let $\varphi^{-}:=\neg \varphi$ for arithmetic formulas.

Definition 20.1 (in $\mathbf{L}$ ). We define a relation $\mathbf{T}$ forc $\varphi$ between multitrees $\mathbf{T} \in$ MT and closed normal $\mathscr{L}$-formulas:

(I) if $\varphi$ is a closed $\mathscr{L}$-formula, arithmetic or in $\mathscr{L} \Sigma_{1}^{1} \cup \mathscr{L} \Pi_{1}^{1}$, and $|\varphi| \subseteq B=|\mathbf{T}|$, then $\mathbf{T}$ forc $\varphi$ whenever $\varphi[x]$ holds for all $x \in[\mathbf{T}]$;

(II) if $\varphi:=\exists x \psi(x)$ is a closed $\mathscr{L} \Sigma_{n+1}^{1}$-formula, $n \geq 1\left(\psi\right.$ belongs to $\left.\mathscr{L} \Pi_{n}^{1}\right)$, then $\mathbf{T}$ forc $\varphi$ whenever there is a code $\mathbf{c} \in \mathrm{CCF}$ such that $\mathbf{T}$ forc $\psi(\mathbf{c})$;

(III) if $\varphi$ is a closed $\mathscr{L} \Pi_{n}^{1}$-formula, $n \geq 2$, then $\mathbf{T}$ forc $\varphi$ whenever there is no multitree $\mathbf{S} \in \mathbf{M T}$ satisfying $\mathbf{S} \leqslant \mathbf{T}$ and $\mathbf{S}$ forc $\varphi^{-}$.

We define $\boldsymbol{F o r c}(\varphi)=\{T \in \mathbf{S T}: T$ forc $\varphi\}$ and $\operatorname{Des}(\varphi)=\operatorname{Forc}(\varphi) \cup \operatorname{Forc}\left(\varphi^{-}\right)$.

Lemma 20.2 (in $\mathbf{L}$ ). If $m \geq 2$ and $\varphi$ is a closed formula in $\mathscr{L} \Sigma_{m}^{1}$, resp., $\mathscr{L} \Pi_{m}^{1}$, then $\operatorname{Forc}(\varphi)$ belongs to $\Sigma_{m-1}^{\mathrm{HC}}(\mathrm{HC})$, resp., $\Pi_{m-1}^{\mathrm{HC}}(\mathrm{HC})$.

Proof. If $\varphi$ is a $\mathscr{L} \Pi_{1}^{1}$ formula then $\operatorname{Forc}(\varphi) \in \Pi_{1}^{1}$ by Definition 20.1(I), and hence $\operatorname{Forc}(\varphi)$ belongs to $\Delta_{1}^{\mathrm{HC}}(\mathrm{HC})$. Then argue by induction using 20.1(II),(III). 


\section{Auxiliary forcing relation: two lemmas}

We here prove two key properties of the relation forc. They will be used in the proof of Theorem 15.6(ii) below. One of them (Lemma 21.2) says that forc is connected with the truth in $\square$-generic extensions similarly to the ordinary $\square$ forcing - for formulas of cartain complexity. The other one (Lemma 21.3) claims the invariance forc relatively to the permutations of $\omega_{1}$.

Recall that a number $n \geq 3$ is fixed by Definition 15.5.

Lemma 21.1 (in $\mathbf{L})$. Let $\varphi$ be a closed normal $\mathscr{L}$-formula. Then the set $\operatorname{Des}(\varphi)$

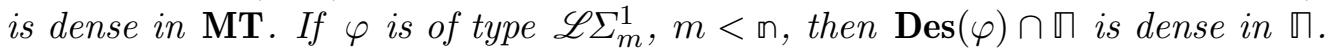

Proof. It suffices to prove the density of $\operatorname{Des}(\varphi)$ for formulas $\varphi$ as in 20.1(I). If $\varphi$ is such and $\mathbf{T} \in \mathbf{M T},|\varphi| \subseteq B=|\mathbf{T}|$, then the set $X(\varphi)=\{x \in[\mathbf{T}]: \varphi[x]\}$ in $\left(2^{\omega}\right)^{B}$ belongs to $\boldsymbol{\Sigma}_{1}^{1} \cup \boldsymbol{\Pi}_{1}^{1}$ and hence has the Baire property inside the closed set $[\mathbf{T}] \subseteq\left(2^{\omega}\right)^{B}$. It remains to refer to Lemma 10.1. The second claim follows by lemmas 20.2 and 16.4 .

Lemma 21.2. Assume that $1 \leq n<\mathrm{n}, \varphi \in \mathbf{L}$ is a closed formula in $\mathscr{L} \Pi_{n}^{1} \cup$ $\mathscr{L} \Sigma_{n+1}^{1}$, and a set $\mathbb{G} \subseteq \mathbb{P}$ is generic over $\mathbf{L}$. Then $\varphi[\boldsymbol{x}[\mathbb{G}]]$ holds in $\mathbf{L}[\mathbb{G}]$, if and only iff $\exists \mathbf{T} \in \mathbb{G}(\mathbf{T}$ forc $\varphi)$.

Proof. Base of induction: $\varphi$ is arithmetic or $\mathscr{L} \Sigma_{1}^{1} \cup \mathscr{L} \Pi_{1}^{1}$, as in 20.1(I). If $\mathbf{T} \in \mathbb{G}$ and $\mathbf{T}$ forc $\varphi$ then $\varphi[\boldsymbol{x}[\mathbb{G}]]$ holds in $\mathbf{L}[\mathbb{G}]$ by the Shoenfield absoluteness theorem, since $\boldsymbol{x}[\mathbb{G}] \uparrow|\mathbf{T}| \in[\mathbf{T}]$. In the opposite direction apply Lemma 21.1.

Step $\mathscr{L} \Pi_{n}^{1} \Longrightarrow \mathscr{L} \Sigma_{n+1}^{1}: \varphi$ is $\exists x \psi(x)$, where $\psi$ belongs to $\mathscr{L} \Pi_{n}^{1}$. Let $\mathbf{T} \in \mathbb{G}$ and $\mathbf{T}$ forc $\varphi$. Then by Definition 20.1(II) there exists a code $\mathbf{c} \in \mathrm{CCF} \cap \mathbf{L}$ such that $\mathbf{T}$ forc $\psi(\mathbf{c})$. By the inductive hypothesis, the formula $\psi(\mathbf{c})[\boldsymbol{x}[\mathbb{G}]]$, i.e., $\psi[\boldsymbol{x}[\mathbb{G}]]\left(f^{\mathbf{c}}(\boldsymbol{x}[\mathbb{G}]\lceil B))\right.$, where $B=|\mathbf{c}|$, holds in $\mathbf{L}[\mathbb{G}]$. Then $\varphi[\boldsymbol{x}[\mathbb{G}]]$ is true as well.

Conversely assume that $\varphi[\boldsymbol{x}[\mathbb{G}]]$ holds in $\mathbf{L}[\mathbb{G}]$. There is a real $y \in \mathbf{L}[\mathbb{G}] \cap 2^{\omega}$ such that $\psi[\boldsymbol{x}[\mathbb{G}]](y)$ holds. By Lemma 17.4,y $=f^{\mathbf{c}}[\mathbb{G}]=f^{\mathbf{c}}(\boldsymbol{x}[\mathbb{G}]\lceil B)$, where $\mathbf{c} \in \mathrm{CCF} \cap \mathbf{L}$ and $B=|\mathbf{c}|$. Then $\psi(\mathbf{c})[\boldsymbol{x}[\mathbb{G}]]$ holds in $\mathbf{L}[\mathbb{G}]$. By the inductive hypothesis, there is $\mathbf{T} \in \mathbb{G}$ such that $\mathbf{T}$ forc $\psi(\mathbf{c})$, hence, $\mathbf{T}$ forc $\varphi$.

Step $\mathscr{L} \Sigma_{n}^{1} \Longrightarrow \mathscr{L} \Pi_{n}^{1}: \varphi$ is a $\mathscr{L} \Pi_{n}^{1}$ formula, $n \geq 2$. Lemma 21.1 yields a multitree $\mathbf{T} \in \mathbb{G}$ such that either $\mathbf{T}$ forc $\varphi$ or $\mathbf{T}$ forc $\varphi^{-}$. If $\mathbf{T}$ forc $\varphi^{-}$then $\varphi^{-}[\boldsymbol{x}[\mathbb{G}]]$ holds in $\mathbf{L}[\mathbb{G}]$ by the inductive hypothesis, hence, $\varphi[\boldsymbol{x}[\mathbb{G}]]$ fails. Now assume that $\mathbf{T}$ forc $\varphi$. We have to prove $\varphi[\boldsymbol{x}[\mathbb{G}]]$ in $\mathbf{L}[\mathbb{G}]$. Suppose to the contrary that $\varphi^{-}[\boldsymbol{x}[\mathbb{G}]]$ holds. By the inductive hypothesis, there exists a multitree $\mathbf{S} \in \mathbb{G}$ such that $\mathbf{S}$ forc $\varphi^{-}$. But the multitrees $\mathbf{S}, \mathbf{T}$ belong to the generic set $\mathbb{G}$, hence, they are compatible, which contradicts to the assumption $\mathbf{T}$ forc $\varphi$.

Invariance. The relation forc turns out to be invariant with respect to the natural action of the group $H$ of all self-inverse (i.e., $h=h^{-1}$ ) permutations of the set $\omega_{1}^{\mathbf{L}}$ in $\mathbf{L}$. Thus $h \in H$, iff $h \in \mathbf{L}, h: \omega_{1}^{\mathbf{L}} \stackrel{\text { onto }}{\longrightarrow} \omega_{1}^{\mathbf{L}}$ is a bijection, and $h=h^{-1}$.

We argue in $\mathbf{L}$. Let $h \in H$. If $B \subseteq \omega_{1}$ and $F$ is a function defined on $B$ then a function $h F=h \cdot F$ is defined on $h " B=\{h(\xi): \xi \in B\}$ so that $(h F)(h(\xi))=F(\xi)$ 
for all $\xi \in B$. Thus $h F$ is equal to the superposition $F \circ h^{-1}$, and even $h F=F \circ h$ by the self-invertibility.

In particular if $x \in\left(2^{\omega}\right)^{B}$ then $h x \in\left(2^{\omega}\right)^{h " B}$, and if $\mathbf{T} \in \mathbf{M T}_{B}$ then $h \mathbf{T}=h \cdot \mathbf{T}$ is a multitree in $\mathbf{M T}_{h " B}$. Further, if $\mathbf{c} \in \mathrm{CCF}_{B}$ then a code $h \mathbf{c}=h \cdot \mathbf{c} \in \mathrm{CCF}_{h}$ "B can be canonically defined so that $f^{h \mathbf{c}}(h x)=f^{\mathbf{c}}(x)$ for all $\xi \in B$. Finally if $\varphi:=$ $\varphi\left(\mathbf{c}_{1}, \ldots, \mathbf{c}_{k}\right)$ is a $\mathscr{L}$-formula then $h \varphi$ or $h \cdot \varphi$ denotes the formula $\varphi\left(h \mathbf{c}_{1}, \ldots, h \mathbf{c}_{k}\right)$. Then $(h \varphi)[h x]$ coincides with $\varphi[x]$.

Lemma 21.3 (in $\mathbf{L}$ ). Let $h \in H, \mathbf{T} \in \mathbf{M T}$, and $\varphi$ is a closed normal $\mathscr{L}$-formula. Then $\mathbf{T}$ forc $\varphi$ if and only if $h \mathbf{T}$ forc $h \varphi$.

Proof. If $\varphi$ is a formula of type $20.1(\mathrm{I})$ then $[h \mathbf{T}]=\{h x: x \in[\mathbf{T}]\}$, and on the other hand, if $x \in[\mathbf{T}]$ then $\varphi[x]$ coincides with $(h \varphi)[h x]$. We skip further routine indictive steps on the base of Definition 20.1(II),(III).

\section{Uniformization of sets with countable sections}

To prove claim (i) of Theorem 15.6 in the end of this section, we establish Theorem 22.1 saying that in $\square$-generic extensions any element of a countable $\boldsymbol{\Sigma}_{\mathrm{m}}^{1}$ set $X$ is constructible relative to the parameter of a $\boldsymbol{\Sigma}_{\mathrm{m}}^{1}$ definition of $X$. The relation forc and Lemma 21.2 will play the key role.

Theorem 22.1. If a set $\mathbb{G} \subseteq \mathbb{P}$ is $\mathbb{P}$-generic over $\mathbf{L}$ and $p \in \mathbf{L}[\mathbb{G}] \cap 2^{\omega}$, then it is true in $\mathbf{L}[\mathbb{G}]$ any countable $\Sigma_{\mathfrak{n}}^{1}(p)$ set $Y \subseteq 2^{\omega}$ satisfies $Y \subseteq \mathbf{L}[p]$.

In fact a stronger claim $Y \in \mathbf{L}[p]$ holds. However it requires more complex transformations beyond $H$, so we are going to skip this issue whatsoever.

Proof. We argue in terms of Definition 15.5. Suppose to the contrary that $Y \nsubseteq$ $\mathbf{L}[p]$. Then $Y=\left\{y \in 2^{\omega}: \varphi(p, y)\right\}$, where $\varphi(p, y):=\exists z \psi(p, y, z)$ is a $\Sigma_{\mathrm{m}}^{1}$ formula with $p$ as the only parameter, and there is a real $y_{0} \in Y, y_{0} \notin \mathbf{L}[p]$. By Theorem 17.5, there is an ordinal $\eta<\omega_{1}^{\mathbf{L}}$ such that $p \in \mathbf{L}\left[\boldsymbol{x}[\mathbb{G}]\left\lceil C_{\neq \eta}\right]\right.$ and $\boldsymbol{x}_{\eta}[\mathbb{G}] \in \mathbf{L}\left[y_{0}\right]$, and moreover, $\boldsymbol{x}_{\eta}[\mathbb{G}]=g\left(y_{0}\right)$, where $g: 2^{\omega} \rightarrow 2^{\omega}$ is a continuous map coded in $\mathbf{L}$. By Lemma 17.4, there exist codes $\mathbf{c}, \mathbf{d} \in \mathrm{CCF}$ satisfying $p=f^{\mathbf{d}}[\mathbb{G}]=f^{\mathbf{d}}(\boldsymbol{x}[\mathbb{G}]\lceil B)$ and $y_{0}=f^{\mathbf{c}}[\mathbb{G}]=f^{\mathbf{c}}\left(\boldsymbol{x}[\mathbb{G}] \mid B^{\prime}\right)$, where $B=|\mathbf{d}| \subseteq C_{\neq \eta}$ and $B^{\prime}=|\mathbf{c}|$. We can assume that $B \subseteq B^{\prime}$ and $\eta \in B^{\prime}$. Note that definitely $\eta \notin B$.

The goal is to get a contradiction.

Consider the $\mathscr{L} \Sigma_{\mathbb{m}}^{1}$ formula $\varphi(\mathbf{d}, \mathbf{c})$. By the choice of the codes, $\varphi(\mathbf{d}, \mathbf{c})[\boldsymbol{x}[\mathbb{G}]]$ coincides with $\varphi\left(f^{\mathbf{d}}[\mathbb{G}], f^{\mathbf{c}}[\mathbb{G}]\right)$, therefore $\varphi(\mathbf{d}, \mathbf{c})[\boldsymbol{x}[\mathbb{G}]]$ holds in $\mathbf{L}[\mathbb{G}]$. By Lemma 21.2 , there is a multitree $\mathbf{S} \in \mathbb{G}$ satisfying $\mathbf{S}$ forc $\varphi(\mathbf{d}, \mathbf{c})$.

Further, the equality $\boldsymbol{x}_{\eta}[\mathbb{G}]=g\left(y_{0}\right)$ (see above) can be rewritten as $f^{\mathbf{e}}\left(\boldsymbol{x}[\underline{G}]\left\lceil B^{\prime}\right)=g\left(f^{\mathbf{c}}\left(\boldsymbol{x}[\mathbb{G}]\left\lceil B^{\prime}\right)\right)\right.\right.$, where $\mathbf{e} \in \mathrm{CCF}_{B^{\prime}} \cap \mathbf{L}$ is a canonical code of the map $f^{\mathbf{e}}(x)=x(\eta)$. We render this formula as

$$
\exists z\left(z=f^{\mathbf{c}}\left(\boldsymbol{x}[\mathbb{G}]\left\lceil B^{\prime}\right) \wedge f^{\mathbf{e}}\left(\boldsymbol{x}[\underline{G}]\left\lceil B^{\prime}\right)=g(z)\right) .\right.\right.
$$


As above by Lemma 21.2 there exists a multitree $\mathbf{S}^{\prime} \in \mathbb{G}$ satisfying $\mathbf{S}^{\prime}$ forc $\exists z(z=$ $\mathbf{c} \wedge \mathbf{e}=g(z)$ ). We can assume that $\mathbf{S}^{\prime}=\mathbf{S}$. (Otherwise replace both multitrees by a stronger multitree in $\mathbb{G})$. Thus we have

$(*) \mathbf{S}$ forc $\varphi(\mathbf{d}, \mathbf{c})$ and $\mathbf{S}$ forc $\exists z(z=\mathbf{c} \wedge \mathbf{e}=g(z))$.

We can wlog assume that $|\mathbf{S}|=B^{\prime}$, as otherwise we just replace $B^{\prime}$ by $B^{\prime} \cup|\mathbf{S}|$ and $\mathbf{S}$ by $\mathbf{S} \uparrow\left(B^{\prime} \cup|\mathbf{S}|\right)$.

If $\vartheta<\omega_{1}^{\mathbf{L}}$ then let $H_{\vartheta}$ denote the set of all permutations $h \in H$ such that $h(\xi)=\xi$ for all $\xi \in B$ and $h(\xi)>\vartheta$ for all $\xi \in B^{\prime} \backslash B$.

Lemma 22.2. If $\vartheta<\omega_{1}^{\mathbf{L}}$ then there is a permutation $h \in H_{\vartheta}$ and a multitree $\mathbf{S}^{\prime} \in \mathbb{G}$ such that $\mathbf{S}^{\prime} \leqslant h \cdot \mathbf{S}$. (It is not assumed that $h \cdot \mathbf{S} \in \mathbb{T}$.)

Proof (Lemma). Arguing in $\mathbf{L}$, consider the set $\mathbf{D}_{\vartheta}$ of all multitrees $\mathbf{S}^{\prime} \in \mathbf{M T}$ such that $\mathbf{S}^{\prime} \leqslant \mathbf{S}$ and there exists a permutation $h \in H_{\vartheta}$ such that the multitree $h \cdot \mathbf{S}$ satisfies $\mathbf{S}^{\prime} \leqslant h \cdot \mathbf{S}$. A routine estimation shows that $\mathbf{D}$ is a $\Sigma_{1}^{\mathrm{HC}}(\mathbf{S}, \vartheta)$ set. Therefore by Lemma 16.4 there is a multitree $\mathbf{S}^{\prime} \in \mathbb{G}$, such that either (1) $\mathbf{S}^{\prime} \in \mathbf{D}_{\vartheta}$, or (2) there is no multitree $\mathbf{R} \in \mathbf{D}_{\vartheta}$ satisfying $\mathbf{R} \leqslant \mathbf{S}^{\prime}$. And as $\mathbf{S}$ also belongs to $\mathbb{G}$, we cal wlog assume that $\mathbf{S}^{\prime} \leqslant \mathbf{S}$.

We claim that (2) is impossible. Indeed let $\gamma<\omega_{1}^{\mathbf{L}}$ satisfy $\left|\mathbf{S}^{\prime}\right| \subseteq \gamma$ and $\gamma \geq \vartheta$. Define a permutation $h$ by $h(\xi)=\xi$ for $\xi \in B, h(\xi)=h^{-1}(\xi)=\gamma+\xi$ for $\xi<\gamma$, $\xi \notin B$, and still $h(\xi)=\xi$ for all other $\xi<\omega_{1}^{\mathbf{L}}$. The multitrees $\mathbf{S}^{\prime}$ and $\mathbf{U}=h \cdot \mathbf{S}^{\prime}$ coincide on the common domain $\left|\mathbf{S}^{\prime}\right| \cap|\mathbf{U}|=B$, hence are compatible. It follows that the union $\mathbf{R}=\mathbf{S}^{\prime} \cup \mathbf{U}$ belongs to $\mathbf{M T}$ and $\mathbf{R} \leqslant \mathbf{S}^{\prime}, \mathbf{U}$. And further we have $\mathbf{R} \leqslant \mathbf{U}=h \cdot \mathbf{S}^{\prime} \leqslant h \cdot \mathbf{S}$ by construction, hence $\mathbf{R} \in \mathbf{D}$, as required. Thus (2) fails. Therefore (1) holds, that is, $\mathbf{S}^{\prime} \in \mathbf{D}_{\vartheta}$, as required.

(Lemma)

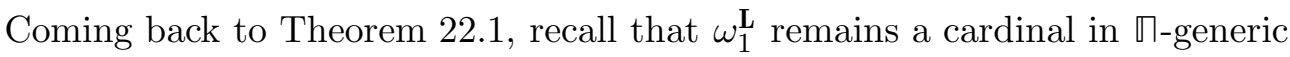
extensions by Lemma 16.3. Therefore Lemma 22.2 allows to define by induction an increasing sequence $\left\langle\vartheta_{\nu}\right\rangle_{\nu<\omega_{1}^{\mathbf{L}}}$ of ordinals $\vartheta_{\nu}<\omega_{1}^{\mathbf{L}}$ and a sequence of multitrees $\mathbf{S}_{\nu} \in \mathbb{G}$ and a sequence of permutations $h_{\nu} \in H_{\vartheta_{\nu}}$, satisfying $B^{\prime} \subseteq \vartheta_{0}$ and $\mathbf{S}_{\nu} \leqslant$ $h_{\nu} \cdot \mathbf{S}$ for all $\nu$, and $\left|\mathbf{S}_{\mu}\right| \subseteq \vartheta_{\nu}$ for $\mu<\nu$.

Let $\mathbf{T}_{\nu}=h_{\nu} \cdot \mathbf{S}, \mathbf{c}_{\nu}=h_{\nu} \cdot \mathbf{c}, \mathbf{d}_{\nu}=h_{\nu} \cdot \mathbf{d}, \mathbf{e}_{\nu}=h_{\nu} \cdot \mathbf{e}$ for all $\nu$. Then we have $\mathbf{T}_{\nu}$ forc $\varphi\left(\mathbf{d}_{\nu}, \mathbf{c}_{\nu}\right)$ and $\mathbf{T}_{\nu}$ forc $\exists z\left(z=\mathbf{c}_{\nu} \wedge \mathbf{e}_{\nu}=g(z)\right)$ by $(*)$ and lemma 21.3. It follows that

$(\dagger) \quad \mathbf{S}_{\nu}$ forc $\varphi\left(\mathbf{d}, \mathbf{c}_{\nu}\right)$ and $\mathbf{S}_{\nu}$ forc $\exists z\left(z=\mathbf{c}_{\nu} \wedge \mathbf{e}_{\nu}=g(z)\right)$,

since $\mathbf{S}_{\nu} \leqslant \mathbf{T}_{\nu}$, and, with respect to the code $\mathbf{d}: \mathbf{d}_{\nu}=h_{\nu} \cdot \mathbf{d}=\mathbf{d}$. (Indeed $h_{\nu}(\xi)=\xi$ whenever $\xi \in B=|\mathbf{d}|$.)

Recall that $f^{\mathbf{d}}\left(\boldsymbol{x}[\mathbb{G}]\lceil B)=p\right.$. Let $B_{\nu}^{\prime}=h^{\prime \prime} B^{\prime}, z_{\nu}=f^{\mathbf{c}_{\nu}}\left(\boldsymbol{x}[\mathbb{G}]\left\lceil B_{\nu}^{\prime}\right)\right.$, and $q_{\nu}=f^{\mathbf{e}_{\nu}}\left(\boldsymbol{x}[\mathbb{G}]\left\lceil B_{\nu}^{\prime}\right)\right.$. If $\nu<\omega_{1}^{\mathbf{L}}$ then, by $(\dagger)$ and Lemma 21.2, $\varphi\left(p, z_{\nu}\right)$ holds in $\mathbf{L}[\mathbb{G}]$ - hence $z_{\nu} \in Y$, and we have $q_{\nu}=g\left(z_{\nu}\right)$ as well. Further,

$$
\begin{aligned}
q_{\nu} & =f^{\mathbf{e}_{\nu}}\left(\boldsymbol{x}[\mathbb{G}]\left\lceil B_{\nu}^{\prime}\right)=\left(h_{\nu} \cdot f^{\mathbf{e}}\right)\left(\boldsymbol{x}[\mathbb{G}]\left\lceil B_{\nu}^{\prime}\right)=f^{\mathbf{e}}\left(h_{\nu}^{-1}\left(\boldsymbol{x}[\mathbb{G}]\left\lceil B_{\nu}^{\prime}\right)\right)\right.\right.\right. \\
& =\quad f^{\mathbf{e}}\left(\left(h_{\nu}^{-1}(\boldsymbol{x}[\mathbb{G}])\left\lceil B^{\prime}\right)=\left(h_{\nu}^{-1}(\boldsymbol{x}[\mathbb{G}])\right)(\eta)=(\boldsymbol{x}[\mathbb{G}])\left(\eta_{\nu}\right)=\boldsymbol{x}_{\eta_{\nu}}[\mathbb{G}]\right.\right.
\end{aligned}
$$


where $\eta_{\nu}=h_{\nu}(\eta)$. Thus an uncountable sequence of the reals $z_{\nu} \in Y$ in $\mathbf{L}[\mathbb{G}]$ $\left(\nu<\omega_{1}^{\mathbf{L}}\right)$ is defined, and it satisfies $g\left(z_{\nu}\right)=\boldsymbol{x}_{\eta_{\nu}}[\mathbb{G}], \forall \nu$. The ordinals $\eta_{\nu}=h_{\nu}(\eta)$ satisfy $\eta_{\nu} \geq \vartheta_{\nu}$ by the choice of $h_{\nu}$, since $\eta \in B^{\prime} \backslash B$. Therefore there exist uncountably many pairwise different of $\eta_{\nu}$ in $\mathbf{L}[\mathbb{G}]$. It follows that there exist uncountably many pairwise different generic reals $\boldsymbol{x}_{\eta_{\nu}}[\mathbb{G}]$. On the other hand, all reals $z_{\nu}$ belong to the countable set $Y$, and $\boldsymbol{x}_{\eta_{\nu}}[\mathbb{G}]=g\left(z_{\nu}\right)$, where $g$ does not depend on $\nu$. This is a contradiction required, and the theorem is proved.

Proof (Theorem 15.6(ii)). Arguing under the requirements of Therorem 15.6, assume that, in $\mathbf{L}[\mathbb{G}], p \in 2^{\omega}$ and $W \subseteq 2^{\omega} \times 2^{\omega}$ is a $\Sigma_{\mathrm{m}}^{1}(p)$ set whose cross-sections $W_{x}=\{y:\langle x, y\rangle \in W\}$ are at most countable. Every set $W_{x}$ is $\Sigma_{\mathrm{m}}^{1}(p, x)$, whence $W_{x} \subseteq \mathbf{L}[p, x]$ by Theorem 22.1. If $W_{x} \neq \varnothing$ then let $q_{x}$ be the $<_{p x}$-least real in $W_{x}$, where $<_{p x}$ is the canonical Gödel well-ordering of $\mathbf{L}[p, x]$. The set $Q=\left\{\left\langle x, q_{x}\right\rangle\right.$ : $\left.x \in 2^{\omega} \wedge W_{x} \neq \varnothing\right\}$ then uniformizas $W$. Moreover

$$
\langle x, y\rangle \in Q \Longleftrightarrow\langle x, y\rangle \in W \wedge \forall z\left(z<_{p x} y \Longrightarrow\langle x, y\rangle \notin W\right) .
$$

Therefore the set $Q$ belongs to $\Delta_{\mathrm{m}+1}^{1}(p)$, or more exactly is the intersection of a $\Sigma_{\text {๓ }}^{1}(p)$ set and a $\Pi_{\mathfrak{n}}^{1}(p)$ set, because the Gödel well-orderings $<_{p x}$ are well-known to be $\Sigma_{2}^{1}(p, x)$-definable uniformly in $p, x$.

(Theorems 2.2 and 2.1)

\section{References}

[1] J.W. Addison. Separation principles in the hierarchies of classical and effective descriptive set theory. Fundam. Math., 46:123-135, 1959.

[2] J.W. Addison. Some consequences of the axiom of constructibility. Fundam. Math., 46:337-357, 1959.

[3] Jon Barwise, editor. Handbook of mathematical logic, volume 90 of Studies in Logic and the Foundations of Mathematics. North-Holland, Amsterdam, New York, Oxford, 1978.

[4] Andrés Eduardo Caicedo and Ralf Schindler. Projective well-orderings of the reals. Arch. Math. Logic, 45(7):783-793, 2006.

[5] Clinton T. Conley. Canonizing relations on nonsmooth sets. J. Symb. Log., 78(1):101$112,2013$.

[6] Vera Fischer, Sy David Friedman, and Yurii Khomskii. Measure, category and projective wellorders. J. Log. Anal., 6:1-25, 2014.

[7] Vera Fischer, Sy David Friedman, and Lyubomyr Zdomskyy. Cardinal characteristics, projective wellorders and large continuum. Ann. Pure Appl. Logic, 164(7-8):763-770, 2013.

[8] M. Golshani, V. Kanovei, and V. Lyubetsky. A Groszek - Laver pair of undistinguishable $\mathrm{E}_{0}$ classes. Mathematical Logic Quarterly, 63(1-2):19-31, 2017. 
[9] J. Hadamard, R. Baire, H. Lebesgue, and E. Borel. Cinq lettres sur la théorie des ensembles. Bull. Soc. Math. Fr., 33:261-273, 1905.

[10] Kai Hauser and Ralf-Dieter Schindler. Projective uniformization revisited. Ann. Pure Appl. Logic, 103(1-3):109-153, 2000.

[11] Thomas Jech. Set theory. Springer-Verlag, Berlin-Heidelberg-New York, The third millennium revised and expanded edition, 2003.

[12] Ronald Jensen. Definable sets of minimal degree. In Yehoshua Bar-Hillel, editor, Math. Logic Found. Set Theory, Proc. Int. Colloqu., Jerusalem 1968, pages 122-128. North-Holland, Amsterdam-London, 1970.

[13] V. Kanovei and V. Lyubetsky. A definable $\mathrm{E}_{0}$-class containing no definable elements. Archive for Mathematical Logic, 54(5):711-723, 2015.

[14] V. Kanovei and V. Lyubetsky. Counterexamples to countable-section $\Pi_{2}^{1}$ uniformization and $\Pi_{3}^{1}$ separation. Annals of Pure and Applied Logic, 167(4): 262-283, 2016.

[15] V. Kanovei and V. Lyubetsky. Countable OD sets of reals belong to the ground model. Arch. Math. Logic, to appear., 2018. First Online 24 June 2017, https://doi.org/10.1007/s00153-017-0569-0.

[16] V. Kanovei and V. Lyubetsky. Non-uniformizable sets of second projective level with countable cross-sections in the form of Vitali classes. Izvestiya: Mathematics, 82(1):65-96, 2018. To appear.

[17] V. G. Kanovei. The projective hierarchy of N. N. Luzin: The current state-of-the-art in the theory. In Handbook of Mathematical Logic, Part II: Set Theory, Supplement to the Russian translation, pages 273-364. Nauka, Moscow, 1982. [In Russian.].

[18] V. G. Kanovei and V. A. Lyubetsky. On some classical problems in descriptive set theory. Russ. Math. Surv., 58(5):839-927, 2003.

[19] V. G. Kanovei and V. A. Lyubetsky. Modern Set Theory: Basics of Descriptive Dynamics. Nauka, Moscow, 2007. [In Russian].

[20] V. G. Kanovei and V. A. Lyubetsky. Modern Set Theory: Borel and Projective Sets. MTsNMO, Moscow, 2010. [In Russian].

[21] V. G. Kanovei and V. A. Lyubetsky. Modern Set Theory: Absolutely Unsolvable Classical Problems. MTsNMO, Moscow, 2013. [In Russian].

[22] V. G. Kanovei and V. A. Lyubetsky. A countable definable set containing no definable elements. Mathematical Notes, 102(3-4):338-349, 2017.

[23] Vladimir Kanovei. Non-Glimm-Effros equivalence relations at second projective level. Fundam. Math., 154(1):1-35, 1997.

[24] Vladimir Kanovei. On non-wellfounded iterations of the perfect set forcing. J. Symb. Log., 64(2):551-574, 1999.

[25] Vladimir Kanovei. Borel equivalence relations. Structure and classification. Providence, RI: American Mathematical Society (AMS), 2008.

[26] Vladimir Kanovei, Marcin Sabok, and Jindřich Zapletal. Canonical Ramsey theory on Polish spaces. Cambridge: Cambridge University Press, 2013. 
[27] M. Kondô. L'uniformisation des complémentaires analytiques. Proc. Imp. Acad. Tokyo, 13:287-291, 1937.

[28] Azriel Levy. Definability in axiomatic set theory II. In Yehoshua Bar-Hillel, editor, Math. Logic Found. Set Theory, Proc. Int. Colloqu., Jerusalem 1968, pages 129-145. North-Holland, Amsterdam-London, 1970.

[29] N. Lusin. Sur le problème de M. J. Hadamard d'uniformisation des ensembles. $C$. R. Acad. Sci., Paris, 190:349-351, 1930.

[30] N. Lusin. Sur le problème de M. Jacques Hadamard d'uniformisation des ensembles. Mathematica, Cluj, 4:54-66, 1930.

[31] N. Lusin and P. Novikoff. Choix effectif d'un point dans un complémentaire analytique arbitraire, donné par un crible. Fundamenta Math., 25:559-560, 1935.

[32] N. N. Luzin. Work Collect, volume II. USSR Academy of Science, Moscow, 1958.

[33] Yiannis N. Moschovakis. Descriptive set theory. Studies in Logic and the Foundations of Mathematics, Vol. 100. Amsterdam, New York, Oxford: North-Holland Publishing Company. XII, 637 p. Dfl. 150.00; \$ 73.25, 1980.

[34] J.P. Ressayre. $\Pi_{2}^{1}$-logic and uniformization in the analytical hierarchy. Arch. Math. Logic, 28(2):99-117, 1989.

[35] R. M. Solovay. A model of set-theory in which every set of reals is lebesgue measurable. Ann. Math. (2), 92:1-56, 1970.

[36] V.A. Uspenskij. Luzin's contribution to the descriptive theory of sets and functions: concepts, problems, predictions. Russ. Math. Surv., 40(3):97-134, 1985.

[37] W.Hugh Woodin. On the consistency strength of projective uniformization. Logic colloquium '81, Proc. Herbrand Symp., Marseille 1981, Stud. Logic Found. Math. 107, 365-384, 1982. 


\section{Index}

action

$h F, 34$

$h \varphi, 35$

base

$|\mathbf{P}|, 15$

$|\mathbf{T}|, 10$

blocks, 20, 25

negative, 25

positive, 25

brick, 10

code

$|\mathbf{c}|, 19$

$\mathrm{CCF}_{B}, 19$

CCF, 19

code of a continuous map, 18

collage, 9,16

definability classes

$\Sigma_{n}^{\mathrm{HC}}(\mathrm{HC}), \Pi_{n}^{\mathrm{HC}}(\mathrm{HC}), 25$

$\Sigma_{n}^{\mathrm{HC}}, \Pi_{n}^{\mathrm{HC}}, \Delta_{n}^{\mathrm{HC}}, 25$

$\mathrm{E}_{0}$-invariant, 4,8

equivalence class

$$
[x]_{\mathrm{E}_{0}}, 4
$$

equivalence relation

class $[x]_{\mathrm{E}_{0}}, 4$

$\mathrm{E}_{0}, 4,6$

$\equiv_{\mathrm{E}_{0}}, 4$

extension

$\mathfrak{M}$-extension, $P \sqsubset_{\mathfrak{M}} Q, 9$

extension, $P \sqsubset Q, 9$

forcing

『, 26

पा $C, 26$

$\square_{C}, 26$

LT-forcing, 8

regular, 8

Cohen, $P_{\text {coh }}, 9$

Cohen, $\mathfrak{S}_{\mathrm{coh}}^{B}, 15$ extension, $P \sqsubset Q, 9$

Jensen forcing, 3

$\mathfrak{M}$-extension $P \sqsubset_{\mathfrak{M}} Q, 9$

restricted, $\square \uparrow C, 26$

restricted, $\square_{C}, 26$

$\mathbb{P}, 26$

formula

$\mathscr{L} \Sigma_{n}^{1}, \mathscr{L} \Pi_{n}^{1}, 33$

$\varphi[x], 33$

$\varphi^{-}, 33$

arithmetic, 33

language $\mathscr{L}, 33$

normal, 33

generic real

$x[G], 8$

hereditarily countable sets, HC, 20

hereditarily countable, $\mathrm{HC}, 25$

invariance

permutation, 34

invariant

$\mathrm{E}_{0}$-invariant, 4,8

map

$f^{\mathbf{c}}, 19$

code, 18

simple, 17

$\mathfrak{M}$-extension of multiforcing, $\sqsubset_{\mathfrak{M}}, 19$

$\mathfrak{M}$-extension of multiforcing, $\sqsubset_{\mathfrak{M}}^{+}, 24$

model

$\mathfrak{M}(\overrightarrow{\mathbf{P}}), 24$

$\mathfrak{M}_{\alpha}, 26$

multiforcing, 15

$|\mathbf{P}|, 15$

$\mathbb{P}_{\alpha}, 26$

$\mathbb{P}_{<\alpha}, 26$

$\mathbf{M T}(\mathbf{P}), 15$

$\mathbb{P}(\xi), 26$

$\mathbb{P}_{\alpha}(\xi), 26$

extension, 19, 24 
regular, 15

small, 15

multitree, 10

$|\mathbf{T}|, 10$

$\Lambda, 10$

MT, 10

$\mathbf{T}^{\uparrow}, 20$

collage, 16

stronger, 10

multitrees

$\mathbf{M T}(\mathbf{P}), 15$

compatible, 15

ordering, 10

set

dense, 16

open dense, 16

pre-dense, 16

number $\mathrm{n} \geq 3,25$

number $\mathrm{n} \geq 3,25$

projection, $\uparrow, \uparrow, 10$

set

$[T], 6$

$[\mathbf{T}], 10$

$K=K[\mathbb{G}], 31$

$W, 32$

dense, 16

hereditarily countable, HC, 20, 25

open dense, 16

pre-dense, 16

splitting, 7

$T(\rightarrow i), 7$

$T(\rightarrow s), 7$

stem, $\operatorname{stem}(T), 6$

string, 6

$2^{n}$, strings of length $n, 6$

$\Lambda$, the empty string, 6

action

$$
\begin{aligned}
& s \cdot X, 6 \\
& s \cdot t, 6
\end{aligned}
$$

$s \cdot x, 6$

extension, 6 length, 6

$q_{k}^{i}[T], 6$

submultiforcing, 15

$\mathfrak{S}_{\alpha}, 26$

$\mathfrak{S}(\mathbf{P}), 19$

$\mathfrak{S}^{\uparrow}, 20$

system, 20

$\operatorname{dom} \varphi, 20$

$\operatorname{Sys}\left(\mathfrak{S}^{\uparrow}\right), 20$

extension, $\psi \preccurlyeq \varphi, 20$

theory

$\mathbf{Z F C}^{\prime}, 19$

tree, 6

branch, 6

large, LT, 6

perfect, $\mathbf{P T}, 6$

stem, $\operatorname{stem}(T), 6$

truncated, $T \uparrow_{u}, 6$

$T[s], 7$

$2^{<\omega}, 6$

$C_{\neq \xi}, 26$

CCF, 19

$\mathrm{CCF}_{B}, 19$

$|\mathbf{c}|, 19$

$\bigcup^{\mathrm{cw}}, 24$

DEF, 20

$D[\sigma, \tau], 10$

$\mathrm{E}_{0}, 4,6$

$\mathrm{E}_{0}$-invariant, 4,8

$[x]_{\mathrm{E}_{0}}, 4$

$\equiv_{\mathrm{E}_{0}}, 4$

$f^{\mathbf{c}}, 19$

$\varphi^{-}, 33$

$\operatorname{dom} \varphi, 20$

$\varphi[x], 33$

$\mathbb{G}(\xi), 29$

HC, 20

$\mathrm{HC}, 25$

$K=K[\mathbb{G}], 31$

$\Lambda$, the empty string, 6

$\operatorname{lh}(s)$, length, 6

$\mathscr{L} \Pi_{n}^{1}, 33$ 


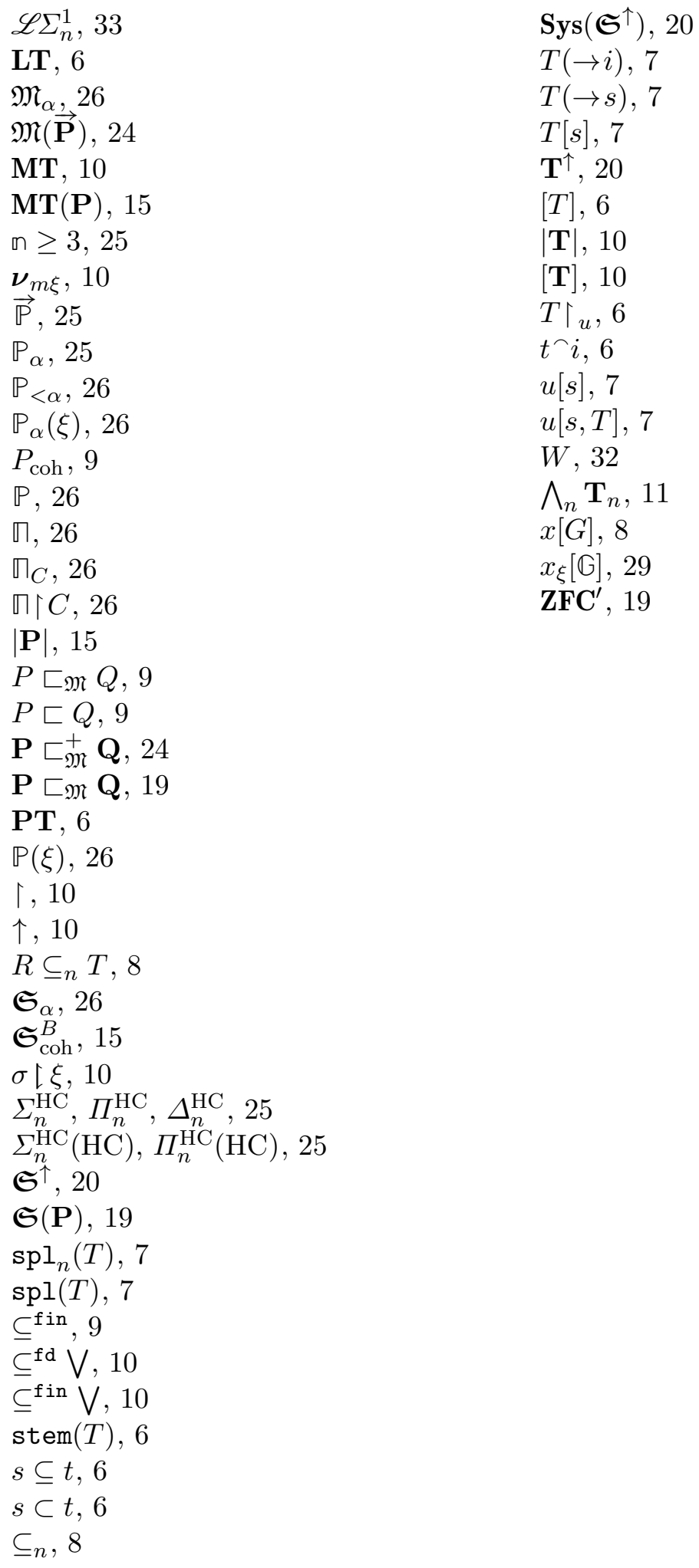

$\subseteq_{n}, 8$ 